\title{
Distracted from sleep : chronic sleep onset insomnia in children with attention-deficit/hyperactivity disorder
}

Citation for published version (APA):

van der Heijden, K. B. (2006). Distracted from sleep : chronic sleep onset insomnia in children with attention-deficit/hyperactivity disorder. [Doctoral Thesis, Maastricht University]. Universiteit Maastricht. https://doi.org/10.26481/dis.20060621kh

Document status and date:

Published: 01/01/2006

DOI:

$10.26481 / \mathrm{dis} .20060621 \mathrm{kh}$

Document Version:

Publisher's PDF, also known as Version of record

\section{Please check the document version of this publication:}

- A submitted manuscript is the version of the article upon submission and before peer-review. There can be important differences between the submitted version and the official published version of record.

People interested in the research are advised to contact the author for the final version of the publication, or visit the DOI to the publisher's website.

- The final author version and the galley proof are versions of the publication after peer review.

- The final published version features the final layout of the paper including the volume, issue and page numbers.

Link to publication

\footnotetext{
General rights rights.

- You may freely distribute the URL identifying the publication in the public portal. please follow below link for the End User Agreement:

www.umlib.nl/taverne-license

Take down policy

If you believe that this document breaches copyright please contact us at:

repository@maastrichtuniversity.nl

providing details and we will investigate your claim.
}

Copyright and moral rights for the publications made accessible in the public portal are retained by the authors and/or other copyright owners and it is a condition of accessing publications that users recognise and abide by the legal requirements associated with these

- Users may download and print one copy of any publication from the public portal for the purpose of private study or research.

- You may not further distribute the material or use it for any profit-making activity or commercial gain

If the publication is distributed under the terms of Article $25 \mathrm{fa}$ of the Dutch Copyright Act, indicated by the "Taverne" license above, 


\section{Distracted From Sleep}

Chronic sleep onset insomnia in children with attention-deficit/hyperactivity disorder

Kristiaan Boudewijn van der Heijden 



\section{Distracted From Sleep}

\section{Chronic sleep onset insomnia in children with attention-deficit/hyperactivity disorder}

\section{Proefschrift}

ter verkrijging van de graad van doctor aan de Universiteit Maastricht, op gezag van de Rector Magnificus, prof. mr. G.P.M.F. Mols

volgens het besluit van het College van Decanen, in het openbaar te verdedigen

op woensdag 21 juni 2006 om 12:00 uur door

Kristiaan Boudewijn van der Heijden

geboren 14 april 1975 te Delft 


\section{Promotiecommissie}

\section{Promotores}

Prof. dr. J.S.H. Vles

Prof. dr. A.P. Aldenkamp (tevens Kempenhaeghe, Heeze)

Prof. dr. W.B. Gunning (Kempenhaeghe, Heeze)

\section{Copromotor}

Dr. M.G. Smits (Ziekenhuis Gelderse Vallei, Ede)

\section{Beoordelingscommissie}

Prof. dr J. Jolles (voorzitter)

Dr. J.B.A.M. Arends (Kempenhaeghe, Heeze)

Dr. J.H.M. de Groen (Kempenhaeghe, Heeze)

Prof. dr. A.M. Korebrits

Prof. dr. M. Limburg

ISBN-10: 90-9020561-6 / ISBN-13: 978-90-9020561-8

Cover design by: Jasper Middelberg.

Cover photo by: Arthur Leipzig.

Printed by: PrintPartners Ipskamp, Amsterdam, The Netherlands.

(C) Kristiaan B. van der Heijden, Amsterdam, The Netherlands, 2006.

All rights reserved. No part of this thesis may be reproduced, stored in a retrieval system of any nature, or transmitted in any form or by any means, electronic, mechanical, photocopying, recording or otherwise, included a complete or partial transcription, without the prior written permission of the author, application for which should be addressed to the author (Transvaalstraat 86-C, $1092 \mathrm{HP}$, Amsterdam, The Netherlands). 
The research described was financially supported by Foundation De Drie Lichten and the Maarten Kappelle Foundation.

Financial support for the publication of this thesis was generously provided by the Research and Development Committee Kempenhaeghe (Commissie Onderzoek en Ontwikkeling Kempenhaeghe), Dutch Society for Sleep-Wake Research (Nederlandse Vereniging voor Slaap-Waak Onderzoek, NSWO), Faculty of Medicine of Maastricht University, and Maastricht Institute of Brain \& Behavior. 


\section{Contents}

\section{General introduction}

1. Introduction, scope and hypotheses.

2. Sleep-related disorders in attention-deficit/hyperactivity disorder:

a review

II. Etiology of chronic sleep onset insomnia in attention-deficit/ hyperactivity disorder

3. Sleep onset insomnia in attention-deficit/hyperactivity disorder: stimulant use, psychiatric co-morbidity, family history, and other sleeprelated problems

4. Sleep hygiene and actigraphically evaluated sleep characteristics in children with attention-deficit/hyperactivity disorder and chronic sleep onset insomnia

5. Idiopathic chronic sleep onset insomnia in attentiondeficit/hyperactivity disorder: a circadian thythm sleep disorder

6. No evidence to support an association of PER3 clock gene polymorphism with attention-deficit/hyperactivity disorder related idiopathic chronic sleep onset insomnia.

III. Melatonin treatment for chronic sleep onset insomnia in childhood

7. Prediction of melatonin efficacy by pre-treatment dim light melatonin onset in children with idiopathic chronic sleep onset insomnia

8. Childhood chronic sleep onset insomnia and late sleep onset: what's the difference? 
9. Effect of melatonin on sleep, behavior, cognitive performance, and quality of life in children with attention/deficit-hyperactivity disorder and chronic sleep onset insomnia

\section{General discussion}

10. General discussion and implications for health care and future research 119

References 127

Appendices 145

Samenvatting 153

Dankwoord 159

Curriculum Vitae 163

List of publications 165 



\section{General introduction}


- 
Introduction, scope and hypotheses 


\section{Introduction}

Attention-deficit/hyperactivity disorder (ADHD) is one of the most common psychiatric disorders of childhood with an estimated prevalence of 3-5\%. It is characterized by pervasive and persistent inattention, hyperactivity/impulsivity, or both, and has a wide-ranging and lifelong impact on education, family relations, and social life. There have been frequent claims that children with ADHD show increased rates of sleep disturbances, with reported rates of up to fifty percent [20]. One of the most frequently reported sleep disturbances in children with ADHD is chronic sleep onset insomnia, a persistent disability to fall asleep at the desired time in the evening [193].

It is a widely accepted view that a healthy sleep is crucial to children's health and proper development. Experimental studies have shown that in elementary schoolaged children, prolonging the wake-time period for only half an hour (i.e., $30 \mathrm{~min}$. sleep deprivation) can result into substantial performance decrements in cognitive function [71;181]. Furthermore, children with sleep-related disorders showed impairments of growth and failure to thrive [29], increased daytime sleepiness, poor performance at school [27;135], and larger numbers of accidents as compared with normal sleepers [208]. Consequently, in children with ADHD disturbed sleep might increase burden and exacerbate ADHD-related impairments of daytime dysfunction. Hence, the simultaneous presentation of ADHD and sleep-related disorders warrants close attention.

There is a paucity of knowledge on aspects of etiology and treatment of chronic sleep onset insomnia in children with ADHD. The aim of this thesis was to unravel the underpinnings of chronic sleep onset insomnia in children with ADHD and to evaluate the effectiveness and safety of melatonin for the treatment of insomnia. More insight into these topics may ultimately contribute to advances in diagnosis, treatment, or prognosis of ADHD. The following section gives an overview of the scope and hypotheses of the studies in this thesis.

\section{Scope and hypotheses}

Chapter two comprises an extensive review of recent literature on sleep-related disorders in children with ADHD published after the introduction of the current psychiatric classification system (Diagnostic and Statistical Manual of Mental Disorders-Fourth Edition [13]). After the publication of earlier reviews on this topic $[20 ; 51 ; 158]$, several recent studies have shown intriguing outcomes. These are discussed in Chapter two and organized in such a way that the results are useful to clinicians in the pediatric field. 
Chapter three describes a cross-sectional, retrospective study of a number of factors potentially related to chronic sleep onset insomnia in children with ADHD. As far as this is possible by using retrospective parent reports, we studied the influence of psychostimulant treatment on sleep. Psychostimulant treatment is the first-choice treatment for $\mathrm{ADHD}$, however, studies have shown that it can have significant adverse effects on sleep. Additionally, we studied the association of chronic sleep onset insomnia with problem behaviors such as depression, anxiety, and oppositional defiant behavior, since previous studies demonstrated a high prevalence of sleep disturbances among children showing these problem behaviors. We furthermore investigated family history of childhood chronic sleep onset insomnia in order to identify a potential contribution of heritable factors to its pathogenesis.

Some behavioral aspects of ADHD such as hyperactivity or disorganized characteristics might be of influence on the ability to fall asleep. Furthermore, sleep problems in children with $\mathrm{ADHD}$ are sometimes ascribed to parent factors, as research has shown that parenting styles can affect sleep quality [192]. Sleep hygiene comprises all environmental and behavioral factors that precede sleep and may interfere with sleep. Previous studies have shown that inadequate sleep hygiene is associated with deteriorated sleep [30] and that improvement of sleep hygiene increases sleep quality [95]. Therefore, we investigated the role of sleep hygiene in the etiology of chronic sleep onset insomnia in children with ADHD (Chapter four). We expected to find a lower level of sleep hygiene in children with $\mathrm{ADHD}$ and chronic sleep onset insomnia than in ADHD controls without insomnia. Such findings would have important consequences for treatment strategies in health care settings.

In a pilot study including a small sample of children with ADHD and chronic sleep onset insomnia, we found a delayed sleep-wake rhythm and a delayed evening rise of the hormone melatonin. The clock time at which the melatonin concentration starts to rise is called dim light melatonin onset and is considered the most reliable phase marker of the circadian pacemaker rhythm. These preliminary results suggested that chronic sleep onset insomnia in medication-free children with ADHD may be due to an underlying circadian thythm sleep disorder, i.e. that the endogenous rhythm of the central circadian pacemaker is fixed at a later phase than desired. Therefore, we investigated this issue in a large study including 87 medication-free children with ADHD and chronic sleep onset insomnia and 33 ADHD controls without insomnia (Chapter five).

In adults, one type of circadian rhythm sleep disorder was associated with a particular form (polymorphism) of a gene (PER3) of the circadian pacemaker. 
Therefore, we hypothesized that chronic sleep onset insomnia in ADHD was associated with this polymorphism of PER3 as well. Chapter six describes a study in which PER3 was studied in a group of children with ADHD and chronic sleep onset insomnia, all of whom showed extremely delayed values of dim light melatonin onset.

Melatonin can be used as a treatment for various types of insomnia related to a circadian thythm disorder, such as in jet lag [92], shift-work [18;185], blindness [179], and delayed sleep phase syndrome [105;143]. Furthermore, it was effective in insomniac children with mental retardation and neurological disorders [99], and Rett's syndrome [131]. One of the suggested underlying mechanisms of melatonin treatment is its 'chronobiotic' effect to phase-shift circadian thythms [120;122]. The magnitude and direction of its phase-shifting effect are dependent on the timing of intake within the endogenous circadian rhythm. In accordance to the phaseresponse curve of melatonin, we hypothesized that the efficacy of melatonin administered in the early evening could be predicted by the dim light melatonin onset as assessed before commencement of treatment.

Chapter seven covers a meta-analysis of two previously performed randomized, placebo-controlled, double blind trials on melatonin efficacy in elementary school children. The pooling of data resulted in a large sample size of 110 participants that allowed for an analysis of the potential predictive utility of dim light melatonin onset. Chapter eight shows our reply to a comment that we received from a colleague after publication of Chapter seven. The comment expressed concern about the use of melatonin in children based on the argument that sleep problems in children are a culturally based phenomenon and rather an extreme variant of normal behavior than a disorder. Our rebuttal aimed to clarify that, if strict and appropriate criteria of chronic sleep onset insomnia are employed, such as we did in our studies, then treatment with melatonin is justified.

As for medication-free children with ADHD and chronic sleep onset insomnia, no studies had addressed the efficacy and safety of melatonin treatment. Therefore, we performed a large randomized, placebo-controlled, double-blind, multicentre trial to investigate whether melatonin improved objective and subjective measures of sleep, but also whether improvements of sleep resulted into an alleviation of impairments of daytime function, as assessed with objective and subjective measures of behavior, cognitive performance, and quality of life (Chapter nine). 
Sleep-related disorders in attention-deficit/ hyperactivity disorder; a review

Van der Heijden, K.B., Smits, M.G., Gunning, W.B. Sleep-related disorders in attention-deficit/hyperactivity disorder; a review. Clin. Pediatr., 2005, 44: 201-210 (minor textual modification). 


\section{Introduction}

The relationship of attention-deficit/hyperactivity disorder (ADHD) with sleeprelated disorders has received remarkable interest. The comorbidity warrants close attention since sleep-related disorders impinge on the same functional domains as those that are impaired in ADHD, such as sustained attention, inhibition, and working memory. Hence, it was suggested that sleep-related disorders might possibly lead to an aggravation or mimicry of the symptoms of ADHD. A second reason for the interest is that stimulants, the treatment of first choice for $\mathrm{ADHD}$, are notorious for its deleterious effect on sleep. A vast number of studies on the issue of sleep in $\mathrm{ADHD}$ has recently been published, including several reviews [20;51;158] and book chapters [31;49]. However, a clear overview of the recent findings with a focus on the clinical implications is yet missing. The present review discusses studies published after the date of the introduction of DSM-IV [13]. The sleep disorders that have been linked to ADHD will be discussed separately. First, some elementary methodological aspects of sleep assessment will be discussed.

\section{Assessment of sleep}

Polysomnography (PSG) is considered the gold standard in sleep research. It usually records two or more electrophysiological measures such as EEG and EMG. Consequently, PSG enables to asses sleep architecture. PSG is relatively expensive and often requires people to sleep in laboratory settings which is known to potentially change habitual sleep patterns and induce a lower sleep efficiency at the first night of the sleep laboratory measurements: "first-night effect" [127;173]. In practice it is usually not possible to obtain PSG recordings of more than two consecutive nights. Actigraphy assesses physical motion with an actiwatch, a small device with the size of a normal wristwatch, which stores the resulting information. Actigraphy can be performed during many consecutive days and nights. Several studies have indicated that for healthy subjects, the agreement rates between actigraph-based and PSG-based minute by minute sleep-wake scoring are above $90 \%$ [141;182-184;227], however its accuracy to estimate sleep reference times (e.g. sleep onset time, time of awakening) is only moderate. The accuracy of actigraphy declines as the quality and quantity of sleep diminishes, such as for patients with sleep-related disorders [88;113;141], and major depression [101].

Subjective methods of sleep assessment can be obtained directly from subjects themselves by means of a clinical history, sleep questionnaires or sleep logs. In case subjects themselves are not able to supply the information needed, bedpartners or parents are requested to record the information. The validity and reliability of sleep 
questionnaires and sleep logs is debatable. Sleep questionnaires and sleep logs are frequently used for intra-individual comparisons, e.g. to assess treatment effects.

\section{Breath-related sleep disorders}

Breath-related sleep disorders (SDB) (see Table 1) are often associated with behavioral problems that show much similarity to characteristics of ADHD [42;80;148;207]. Although hyperactivity is often shown at referral in children with suspected SDB, it is not displayed more often in children with a PSG confirmed diagnosis of SDB [40]. Children with diagnosed ADHD did not significantly more often show symptoms of SDB when assessed with one or two items of a questionnaire $[50 ; 62 ; 154 ; 193]$. When assessed with a questionnaire comprising six snoring- and SDB-related items, children with ADHD showed elevated scores as compared to psychiatric controls [43]. However, three recent PSG-studies showed that the presence of SDB in children with ADHD was negligible [54;107;150]. PSG-studies demonstrated that complaints of SDB, often expressed by parents of children with ADHD, usually can not be confirmed on investigation [54;150]. Such discrepancies between parental and objective measures have also been found for other sleep problems in ADHD, as will be discussed later. To summarize, attention deficits and behavioral problems can often been found in children with SDB. Children with ADHD often show symptoms of SDB as reported by their parents, however, objective studies did not support that SDB occurs more often in children with ADHD.

Treatment. Adenotonsillectomy is the first line of treatment for SDB, and continuous positive airway pressure is an option for those who are not candidates for surgery or do not respond to surgery (for clinical practice guidelines, see [3]). Treatment of SDB establishes a significant reduction in aggression, inattention, and hyperactivity [8;79;153], and in children with diagnosed ADHD normalizes typical electrophysiological features of ADHD [104]. Case reports have been published of patients in which a diagnosis of ADHD had previously been made, but who could be weaned from methylphenidate after treatment of SDB [145]. Therefore, treatment of SDB in children with ADHD should first be focused on SDB. Stimulant treatment for ADHD is not likely to affect SDB severity [150].

\section{Motor restlessness during sleep}

Before 1980, reports of high nocturnal activity in hyperactive children were so common that the American Psychiatric Association decided to enclose it within the 
Table 1. Clinical features and diagnostic methods of sleep-related disorders associated with ADHD.

\begin{tabular}{lll}
\hline Sleep Disorder & Clinical description & Diagnostic methods \\
\hline $\begin{array}{l}\text { Periodic limb } \\
\text { movement }\end{array}$ & $\begin{array}{l}\text { Repetitive flexions of the toes, feet, legs, 1) Surface electromyographic } \\
\text { thighs, and/or the arms in which recordings; 2) Medical history: }\end{array}$ \\
disorder & electromyographic bursts last 0,5 to 5 diagnosis of PLMD requires \\
$(\boldsymbol{P L M D})[197]$ & $\begin{array}{l}\text { seconds, 5-90 seconds between each impact on either sleep or } \\
\text { movement, in series of 4 or more daytime functioning. } \\
\text { movements during any sleep stage. }\end{array}$
\end{tabular}

Restless legs 1) Desire to move the legs usually syndrome associated with paraesthesias (dis(RLS) agreeable sensations); 2) motor rest[9;197;224] lessness; 3) worsening of symptoms at night; 4) worsening of symptoms at rest relieved by activity; 5) The sensations often lead to insomnia.

1) Medical history; 2) Family history: having a positive family history of RLS is supportive of the diagnosis; 3) PLMD: the presence of PLMD is supportive of the diagnosis.

Breath-related
sleep disorders
(SDB) $[72 ; 198]$

Subtypes: a) obstructive sleep apnea (OSAS), defined as a cessation or near cessation of respiration for a minimum of 10 seconds; b) upper airway resistance syndrome (UARS), defined as a reduction of airflow for a minimum of 10 seconds, and c) snoring.

1) PSG; 2) SDB can be misinterpreted as a limb movement disorder. Therefore, recording and scoring of leg movements must be an integral part of PSG evaluations.

$\mathrm{SDB}$ often leads to sleep fragmentation and excessive daytime sleepiness.

Insomnia Difficulty initiating or maintaining sleep.
$[44 ; 140]$

1) Medical history; 2) Useful adjuncts: sleep logs, actigraphy, self-administered questionnaires, symptom checklist, or psychological screening tests.

$\begin{array}{ll}\text { Excessive } & \text { Subtypes: a) Monosymptomatic excessive } \\ \text { daytime } & \text { daytime sleepiness: manifestation solely } \\ \text { sleepiness [93] } & \text { by excessive daytime sleepiness; b) } \\ & \text { Polysymptomatic excessive daytime } \\ & \text { sleepiness, characterized by excessive } \\ & \text { daytime sleepiness, nocturnal sleep of } \\ & \text { abnormally long duration, and signs of } \\ & \text { sleep drunkenness on awakening. }\end{array}$

1) Medical history; 2) PSG when sleep disturbances are suspected; 3) Severity assessment by means of a multiple sleep latency test (MSI,T), the maintenance of wakefulness test (MWT), the Oxford Sleep Resistance (OSLER) test and several questionnaires. 
Table 1. Clinical features and diagnostic methods of sleep-related disorders associated with ADHD (continued)

\begin{tabular}{lll}
\hline Sleep Disorder & Clinical description & Diagnostic methods \\
$\begin{array}{l}\text { Disturbed } \\
\text { sleep } \\
\text { architecture }\end{array}$ & $\begin{array}{l}\text { Disturbance of sleep architecture: sleep PSG. } \\
\text { stages, cycles, and their inter- } \\
\text { relationships. }\end{array}$ & \\
\hline $\begin{array}{l}\text { Nocturnal } \\
\text { enuresis } \\
{[37 ; 147]}\end{array}$ & $\begin{array}{l}\text { Frequent occurrence of normal complete } \\
\text { uncontrolled micturition during sleep in } \\
\text { children older than five years of age. }\end{array}$ & $\begin{array}{l}\text { The dimary nocturnal enuresis is } \\
\text { limited to the patient's history. }\end{array}$ \\
\hline
\end{tabular}

PSG=Polysomnography

criteria of attention deficit disorder (ADD) in classification system DSM-III [12]. This criterion was omitted in later revisions of the diagnostic manual.

Recent parental observation studies demonstrated that children with ADHD showed higher levels of activity during sleep or had more restless sleep as compared to controls [50;62;148-150;189]. This was confirmed by studies using objective methods such as actigraphy or infrared camera recordings [57;110]. PSG showed a high frequency of short movement-related epochs in children with ADHD, however, the total time of movements was not elevated as compared to normal controls [107]. Motor restlessness during sleep can be a manifestation of a periodic limb movement disorder (PLMD) (see Table 1). Hyperactivity and inattention has been found to be increased in children with PLMD [55]. Another study revealed significant correlation of PLMD-scores with ADHD symptoms, which were both assessed by means of questionnaires [42].

The prevalence of PLMD among children with diagnosed ADHD was significantly higher as compared with normal controls [107;163;164]. However, others found that it is not the number of PLM's per se, but the number of PLM's that are associated with arousals which is increased in children with ADHD. However, the latter applied only to children with ADHD referred to a sleep clinic and not to community samples of children with ADHD [55;149]. Two recent studies showed no increased indexes of PLM's in children with ADHD, but found the highest PLM indexes in the ADHD group [148;150]. However, in these two studies no formal DSM-IV diagnosis of ADHD was made. Studies that investigated the association of ADHD with restless legs syndrome (RLS) (Table 1) found that RLS was associated with symptoms of attention deficit and hyperactivity 
[42], but was not found more frequently among children with a diagnosis of ADHD [43].

In conclusion, there is ample support that children with ADHD show increased motor restlessness during sleep. Whether these movements are manifestations of PLMD/RLS remains unknown due to inconsistency of findings across studies. One of the causes of this inconsistency may have been the difference between studies in assessment of ADHD. Namely, where in some studies only a brief evaluation of symptoms of attention deficit and hyperactivity was applied, in others rigorous diagnostic examinations of $\mathrm{ADHD}$ were carried out.

Stimulant use was found not to be related with PLMD in ADHD [150;163], which makes it unlikely that this factor was involved in the inconsistency of findings. Suggestions were made that ADHD and PLMD might genetically be linked to each other with the dopaminergic system as common factor [110;163], however, this issue needs further examination.

Treatment. Treatment of RLS or PLMD in children with ADHD should start with alleviation of the diseases or deficiencies that may underlie the RLS or PLMD and, in addition, an improvement of sleep hygiene [197]. The most effective and beststudied pharmacological therapy for PLMD/RLS is treatment with dopaminergic agents [45;197]. A study of dopaminergic therapy in seven children with PLMD and comorbid ADHD showed improvements of symptoms of PLMD as well as of ADHD in all patients [225]. The tolerability of dopaminergic agents (modafinil) combined with methylphenidate (eight hours after modafinil) was satisfactory in healthy volunteers [90]. An effect of stimulant treatment on motor restlessness during sleep is unlikely [150].

\section{Insomnia}

It has often been claimed that children with ADHD commonly experience difficulties with settling and falling asleep, which are the main characteristics of insomnia (Table 1). These ideas were supported by most studies using questionnaires that were completed by parents of children with ADHD [50;52;62;137;148;149;154;174;189;193], except for one study [57]. Studies in which parents were asked to record sleep onset, latency, or duration revealed no differences between children with ADHD and controls [82;137;150]. Furthermore, objective measurements of sleep onset time, sleep latency, or sleep duration such as with polysomnography $[110 ; 117 ; 148-150]$ or actigraphy $[52 ; 57 ; 82]$ did not reveal significant differences between children with ADHD and controls. 
To summarize, parents of children with ADHD are more likely to report sleep onset problems compared to parents of children without ADHD. However, a comparison of objective or subjective sleep data between ADHD and control groups does not support this association. Of note, in studies in which parents evaluated their child's sleep both by means of retrospective questionnaire ratings as well as by prospective sleep log measurements, only the retrospective measures revealed a significant association of $\mathrm{ADHD}$ with insomnia [52;62;137;148]. An explanation for the discrepancy could therefore be the relatively high intraindividual variance of sleep onset and offset times found in children with ADHD [81;82]. Averaging the intraindividual sleep data of children with ADHD may therefore mask sleep onset problems present on only several days a week..

Treatment. In American pediatrics, the prescription rate of sleep medication in children with ADHD is two- to four-fold higher than in children without ADHD [155]. The ADHD treatment of first choice with stimulants may deteriorate sleep onset problems [7;22;193;195;202], although one study found no effect on polysomnographically assessed sleep latency and total sleep duration [150]. The efficacy of melatonin treatment for insomnia combined with stimulant treatment for ADHD seems promising and safe [203].

\section{Disturbed sleep architecture}

Sleep architecture disturbances (Table 1) have been studied extensively in children with ADHD primarily before the introduction of DSM-IV. The results demonstrated that children with ADHD showed a shorter rapid eye movement (REM) sleep latency, reduced REM sleep duration, increased delta sleep percentage, as well as a difference in the number of sleep spindles (see [51], p 643, for review). Nevertheless, the findings across the studies were very inconsistent and often in conflict with each other.

Recently, seven polysomnogtaphic studies investigated sleep architecture in children with ADHD. Two studies compared children with ADHD and controls with DSM-IV reading disorders and found no significant differences in sleep architecture [110;117]. One study found a significantly higher mean number of sleep cycles in ADHD [107]. Three other studies found REM sleep duration to be significantly decreased in children with $\mathrm{ADHD}$ as compared to normal controls $[148 ; 149 ; 163]$, while two studies found the opposite results [78;107]. Furthermore, two studies demonstrated that ADHD was associated with an increased REM sleep latency [148;149], whereas one found it related with a decreased REM sleep latency [107]. 
Research has shown that REM sleep disturbances were associated with PLMD [54] as well as with insomnia [219]. REM sleep disturbances were found only in studies that did not exclude comorbid PLMD and insomnia. Hence, a confounding effect of these comorbid factors can not be excluded.

Treatment. The clinical relevance of specific disturbances of sleep architecture remains largely unknown. Treatment options for sleep architecture disturbances are not available. Stimulant treatment for ADHD is not likely to affect sleep architecture [150], however this topic has not been investigated sufficiently.

\section{Nocturnal Enuresis}

Nocturnal enuresis (Table 1) is a common pediatric sleep-related disorder. It often causes distress and impairment of self-esteem [48], however does not result into disturbances of sleep architecture [33;139]. Although the presence of nocturnal enuresis generally increases the risk for psychopathology, this risk seems to be missing in children with ADHD [26].

A vast number of studies have shown increased occurrence rates of nocturnal enuresis in children with ADHD as compared with normal controls $[50 ; 148 ; 149 ; 170 ; 174 ; 176 ; 193]$, and clinical controls [50]. It has been shown that this relationship decreases with advancing age $[170 ; 176]$. Two studies did not find a relationship between ADHD and enuresis [62;137], however, in one of these studies 28 percent of the children with ADHD were using tricyclic antidepressants, which is a class of medicine that has proven effective in the treatment of enuresis.

Both nocturnal enuresis [34;70;222], and ADHD [112;130;204] were shown to be genetically transmittable. However, a recent study has shown that patterns of inheritance of nocturnal enuresis and ADHD are independent of each other [19]. It is of note that most studies on nocturnal enuresis in ADHD are limited in that they failed to make a distinction between primary and secondary nocturnal enuresis, while these subtypes are reasonably distinct in background, prognosis and treatment [223]. In primary enuresis children have never achieved complete nighttime control. Secondary nocturnal enuretics are completely dry at night for a period of at least six months and then begin wetting again. The latter type often occurs after personal or familial disturbance (e.g. school problems) and might therefore be more common among children with ADHD.

To conclude, there is considerable evidence for a link between ADHD and nocturnal enuresis, however, the concomitant presence does not entail additional risk for other psychopathology. 
Treatment. The usual treatment for nocturnal enuresis consists of bladder training, alarm systems or pharmacological agents (tricyclic antidepressants, desmopressin), and usually have success rates of $70-80 \%$. It is unknown whether these treatment options are as effective in ADHD as in children without ADHD. Importantly, tricyclic antidepressants combined with stimulants might lead to abnormal heart rhythms and increased blood pressure. Studies on the effect of stimulants on nocturnal enuresis have yielded very inconsistent tesults [50;150;193].

\section{Excessive daytime sleepiness}

In children, excessive daytime sleepiness (Table 1) may not overtly present as falling asleep during the day or 'sleepy behavior' such as yawning. In fact, sleepy children may instead exhibit hyperactivity, inattention, or behavioral problems $[41 ; 80]$. In childhood excessive daytime sleepiness, somnolence may occur only when the child is not engaged in stimulating mental action or lively physical activity. The multiple sleep latency test measures the ease of falling asleep over the course of a day in a dark laboratory setting and is the gold standard assessment of daytime sleepiness. One study using a multiple sleep latency test in 32 medication-free boys with $\mathrm{ADHD}$ and 22 matched controls found a higher physiological tendency to fall asleep during the day in children with $\mathrm{ADHD}$ as compared with normal controls [117]. These findings were confirmed in a similar recent study using the multiple sleep latency test [78]. Although this study demonstrated that increased somnolence in children with ADHD was not entirely contributable to the presence of SDB or PLMD, it did find an association of PLMD with a significantly shorter sleep latency in the late afternoon. Importantly, both studies found that excessive daytime sleepiness in $\mathrm{ADHD}$ was not related to poorer sleep quality or quantity. Increased sleepiness levels were also found in children with ADHD and/or learning disorder as measured with subjective rating scales [129].

It is yet unknown what mechanisms underlie the suggested association between ADHD and excessive daytime sleepiness. Usually, sleepiness during the day in children is caused by sleep deprivation, sleep fragmentation, and stress from schoolwork [178]. However, the above studies showed that excessive daytime sleepiness in ADHD can not be explained by a reduced sleep quantity or quality or by the presence of concomitant sleep-related disorders. One of the theories behind $\mathrm{ADHD}$ is that the patients suffer from a dysregulation of arousal $[31 ; 78 ; 81 ; 82 ; 117]$. Hence, the elevated levels of daytime sleepiness found in children with ADHD may relate to arousal dysregulation [78]. Also, the increased instability of sleep patterns recently been found in children with ADHD [81;82] might be explained by this dysregulation of arousal. Other studies have underlined the importance of 
arousal regulation in various cognitive functions and have linked it to cognitive dysfunctions in ADHD [21;25]. The importance of arousal dystegulation in ADHD is further corroborated by the beneficial effect of psychostimulant treatment.

Weinberg and Brumback introduced the primary disorder of vigilance (PDV), which is characterized by a combined display of the inability to sustain alertness or wakefulness and decreased attention to current activities [229;230]. They assert PDV can be discerned from ADHD by the presence of a caring, compassionate, affectionate, kind temperament, which is often absent in ADHD.

Treatment. Psychostimulants are the most effective treatment strategy for excessive daytime sleepiness as well as for ADHD. Currently, the majority of children diagnosed with ADHD receive stimulant medication, with rates as high as $85 \%$ in the United States [152].

\section{Conclusions}

The following conclusions may be drawn based on a review of the current literature on sleep-related disorders in children with ADHD.

1. There is high evidence that symptoms of ADHD are more likely to be seen in children with sleep disordered breathing, periodic limb movement disorder, and insomnia.

2. Symptoms of sleep disordered breathing are reported more often by parents of children with ADHD than by parents of children without ADHD. However, there is high evidence that sleep disordered breathing is not more prevalent in ADHD.

3. Increased nocturnal motor restlessness is more often present in ADHD. However, only moderate evidence exists that children with ADHD have a higher risk for periodic limb movements or elevated scores of periodic limb movements as compared to children without ADHD. Restless legs syndrome seems to be related to symptoms of ADHD, but not to ADHD as a diagnosed disorder.

4. Insomnia is more often reported among parents of children with ADHD than among parents of children without ADHD. Further conclusions are hindered due to methodological limitations.

5. There is high evidence that disturbances of sleep architecture can be found in $\mathrm{ADHD}$, however, the nature of sleep architecture disturbances is yet unspecific.

6. There is high evidence that nocturnal enuresis is more likely to occur in ADHD than in children without ADHD.

7. There is limited to moderate evidence that children with ADHD show increased excessive daytime sleepiness. 
8. There is limited evidence that children with ADHD are likely to show unstable sleep patterns.

\section{Clinical implications}

1. Sleep disordered breathing, periodic limb movement disorder, restless legs syndrome, and insomnia may manifest with symptoms of ADHD. A child presenting with symptoms of ADHD should therefore be submitted to a rigorous and careful evaluation of potential sleep problems as part of the clinical history. When sleep related disorders are suspected, subsequent supplementary investigations might be carried out.

2. Clinicians working with children with ADHD should be wary of comorbid insomnia, particularly when the child is treated with stimulants. Knowing the clinical history is important to determine possible predisposing, precipitating, and perpetuating factors. Additional sleep assessment at home can provide more objective information about sleep duration and fragmentation. Sleep assessment should last a week to capture possible sleep pattern instabilities. Discrepancies between findings of the interview and sleep assessment should be discussed with the parents and (if possible) child to identify the cause of the discrepancy.

3. Clinicians should be aware of the relationship between ADHD and nocturnal enuresis and of the possible negative psychological consequences of nocturnal enuresis. Asking for miction problems should be part of the history taking in children with ADHD.

4. Excessive daytime sleepiness can occur covertly in children with ADHD and may only be displayed when the child is not engaged in a stimulating activity. Daytime sleepiness can be a weighing factor in the decision whether or not to prescribe stimulants.

\section{Implications for future studies}

Several methodological issues may be important for future studies. Firstly, the majority of studies discussed are focused on comparison of group means. Another approach is to compare numbers of rates, such as of the presence of a particular disorder, between probands and controls. Such patient-directed approaches yield additional, clinically important, information. Nonetheless, this approach has hardly been used in research on sleep in ADHD. We support a combined use of both methodological approaches in order to reach the highest level of relevant information.

An issue related to this is the absence of consensus criteria on childhood insomnia. In studies of insomnia in children a patient-directed approach is 
hampered due to the lack of consensus criteria on the diagnosis of insomnia in children. Insomnia criteria in the present classification systems are still focused solely on the adult population. Effort should be made to establish age-dependent insomnia criteria for children. Such criteria have been proposed previously [190], however have not been widely implemented yet.

Thirdly, we found that only few studies took into account the composition of their sample with regard to ADHD subtypes (DSM-IV [13]). This in spite of the fact that marked differences in sleep onset, nocturnal movements, sleep fragmentation and efficiency have been found between these ADHD subtypes $[117 ; 166]$. Thus, differences in sample composition between studies might contribute to inconsistencies. Ultimately, taking into account ADHD-subtypes in research might lead to a better understanding of the pathogenesis and treatmenteffectiveness. Therefore, the exploration of potentially new relevant subtypes should be encouraged. A recent attempt has been made by the distinction between "primary (idiopathic) ADHD" and "ADHD secondary to a nocturnal sleep disorder" [78].

A fourth issue pertains to the wide use of actigraphy as an instrument to estimate sleep parameters in ADHD. It is generally considered as a reasonably valid and reliable method to measure sleep-wake differences and has a moderately accuracy to estimate sleep onset and awakening time in normal controls. However, increased nocturnal motor restlessness in ADHD could affect its accuracy to estimate sleep in this population. Hence, concordance of actigraphy and polysomnography measurements should be a topic of further investigation. 


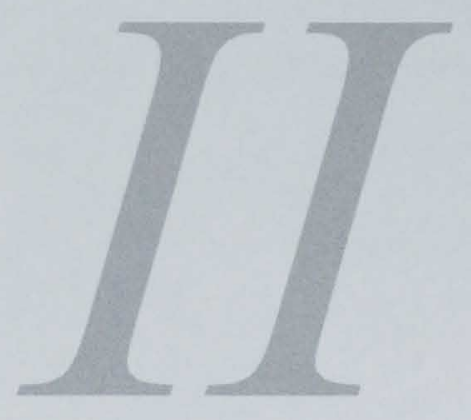

Etiology of chronic sleep onset insomnia in attention-deficit/hyperactivity disorder 

Sleep onset insomnia in attention-deficit/hyperactivity disorder: stimulant use, psychiatric co-morbidity, family history, and other sleep-related problems

Van der Heijden, K.B., Smits, M.G., Veenboer, W., Vlasveld, L., Gerver, J.A.M., Gunning, W.B. Sleep onset insomnia in attention-deficit/hyperactivity disorder: stimulant use, psychiatric co-morbidity, family history, and other sleep-related problems. Submitted. 


\begin{abstract}
Objective. To identify factors related to sleep onset insomnia in children with attention-deficit/hyperactivity disorder (ADHD). Methods. A retrospective parental sleep questionnaire survey in 109 pediatric outpatients (aged 5-15 years) with diagnosed DSM-IV ADHD. Results. Sleep onset insomnia was present in $42.2 \%$ of the children with ADHD (ADHD-SOI), with an estimated sleep onset $( \pm \mathrm{SD}$ ) of 22:00 $\pm 1: 03 \mathrm{~h}$, compared to $20: 43 \pm 1: 04$ $h$ in children with ADHD without insomnia (ADHD-noSOI) $(p<0.001)$. Psychostimulant treatment for ADHD precipitated chronic sleep onset insomnia in twenty percent, and exacerbated existing insomnia in a further fifteen percent. Sleep onset insomnia was significantly related to bedtime resistance $(p<0.0001)$ and co-morbid depressive disorder $(p=0.005)$. Family history of childhood sleep onset insomnia was positive in $39.1 \%$ of ADHD-SOI, compared to $22.2 \%$ in ADHD-noSOI ( $p=0.046)$. Conclusions. The present findings indicate that major factors related to sleep onset insomnia in ADHD are stimulant use, co-morbid depressive disorder, and bedtime resistance. Furthermore, heritable factors may predispose children with ADHD to develop chronic sleep onset insomnia.
\end{abstract}




\section{Introduction}

Attention-deficit/hyperactivity disorder (ADHD) is a syndrome characterized by developmentally inappropriate levels of inattention, impulsiveness, and hyperactivity. There is growing evidence of a link between ADHD and sleep onset insomnia $[50 ; 52 ; 62 ; 137 ; 148 ; 149 ; 154 ; 174 ; 189 ; 193]$. This co-morbidity warrants serious attention since sleep disturbances can exacerbate ADHD-related mood, behavioral, and cognitive problems [145]. As yet, little scientific attention has been focused on the major factors related with sleep onset insomnia in ADHD.

Stimulants are currently the first-choice medication for ADHD but are notorious for their detrimental effect on sleep. Hence, stimulant use may be a risk factor for sleep onset insomnia, however, results across individual studies were relatively inconsistent [50;106;137;150;193]. Another important factor related to insomnia may be a positive family history of insomnia, as has been shown in adults (without ADHD) [60], however, this issue has never been studied in ADHD. Furthermore, there is evidence to suggest that psychiatric disorders co-morbid to ADHD may associate with insomnia. For instance, Corkum et al [50] found that a diagnosis of co-morbid oppositional defiant disorder significantly predicted the presence of dyssomnia symptoms in children with ADHD. In contrast, Mick et al [137] found that the presence of sleep onset insomnia was not related to a diagnosis of oppositional defiant disorder or conduct disorder, but to concomitant anxiety disorder. Correspondingly, a study by Stein [193] showed that internalizing problems were predictive of insomnia in children with ADHD. However, Mick et al. found that concomitant depressive disorder was not associated with insomnia in children with ADHD [137].

The present study is the first systematic study to identify key factors related to sleep onset insomnia in children with diagnosed ADHD. To that end, we investigated stimulant use, psychiatric co-morbidity, family history, and other sleeprelated problems both in children with ADHD and sleep onset insomnia and in ADHD controls without insomnia.

\section{Methods}

\section{Subjects}

The clinical sample of children with ADHD was identified through a chart review of children referred to two Dutch pediatric outpatient clinics in a teaching hospital (Delft) and general hospital (Beverwijk), during the years 2000-2001. Inclusion criteria were: 1) age between 5-15 yrs; 2) diagnosis of ADHD (see below). Presence of psychiatric co-morbidity or stimulant treatment was allowed to facilitate an evaluation of its relationship with sleep onset insomnia. 
The main reasons for referral were: behavioral-, attention-, or learning problems $(95 \%)$; sleep-related problems $(0.9 \%)$; social and communication problems $(0.9 \%)$; depressed mood (0.9\%); or unknown (4.6\%). All children received routine pediatric care after referral. ADHD was carefully diagnosed using criteria of the Diagnostic and Statistical Manual of Mental Disorders, Fourth edition (DSM-IV)[13] by physician-specialists in ADHD (JAMG, LV) who were members of the Dutch board of ADHD pediatricians. Diagnosis was based on behavioral observations, clinical interviews, and administration of standardized rating scales such as the Child Behavior Checklist [6]. All children met diagnostic criteria for severity (six or more DSM-IV criteria), pervasiveness (ADHD symptoms both at home and in school environment), and duration ( $>$ six months) of ADHD symptoms [13]. The study was approved by the institutional ethics boards and written informed consent was obtained from the participating children's parents.

\section{Data collection}

Parents were contacted by mail with information on the study, questionnaire, informed consent form, and return envelope. The initial response rate was $46 \%$. After two mailings, a total of 109 out of 179 questionnaires was returned $(61 \%$ response rate). The prevalence of sleep onset problems in the first responder (i.e. spontaneous) group was similar to that in the second responder (i.e. responder after reminder) group.

\section{Instrument}

The Sleep Questionnaire for Parents of Children with ADHD (Appendix I) was developed by the authors based on a literature review on sleep-related disorders in ADHD [211]. Minor textual modifications were made on the basis of feedback obtained by an evaluation form provided with the first five questionnaires. The final questionnaire consisted of five sections: 1) an ADHD and co-morbidity section, containing specific questions on the diagnosis of ADHD and co-morbid psychiatric disorders on a two-point scale (yes/no) or with open-ended questions (e.g. on date, caregivers); 2) sleep-wake behavior section, including open-ended questions on usual bedtime, estimated sleep onset, rising time, sleeping arrangements, and sleep practices; 3) questions on presence of various sleep-wake problems rated on a three-point scale ("never", "occasionally", "often"); 4) questions regarding the past or present use of sleep medication and/or medication for ADHD (e.g. duration, dose, timing, effect); 5) section on family history of sleep onset insomnia during childhood in first degree relatives (father, mother, sibling). 


\section{Statistics}

Descriptions of the entire sample $(n=109)$ were made by use of frequency, percentage, mean, and standard deviation. The prevalence of sleep-related problems was calculated separately for children with versus children without use of stimulant treatment. Subsequently, the total sample was split into two groups: 1) children with ADHD and sleep onset insomnia (i.e. parents reported that sleep onset problems occurred "often") (ADHD-SOI), and 2) children with ADHD without sleep onset insomnia (i.e. parents reported that sleep onset problems occurred "occasionally" or "never") (ADHD-noSOI). Mean bedtime, sleep latency, and wake up time were calculated for both groups. On the basis of these parameters we calculated sleep onset, defined as the estimated clock time of falling asleep (i.e. bedtime + sleep latency), and total sleep duration, defined as the interval from estimated sleep onset until wake up time (wake up time $+(24: 00 \mathrm{~h}$ - sleep onset)). Independent-samples t-test procedures were employed for between-group comparisons of the estimated sleep measures.

The association between sleep onset insomnia and demographic characteristics (e.g. gender, age), other sleep-related problems, co-morbid psychiatric disorders, use of stimulants, and family history of childhood sleep onset insomnia was analyzed using chi-square $\left(\chi^{2}\right)$ tests or Spearman correlation. Unless stated otherwise, $p$-values are two-sided with statistical significance set at $p<0.05$. In cases where 'unplanned' comparisons were conducted on multiple independent variables, we used a Bonferroni correction, to compensate for the increased probability of a Type I error when multiple interdependent variables were statistically analyzed. The Bonferroni correction was calculated by dividing the chosen alpha by the number of dependent variables. Logistic regression analysis was used to analyze relationship between sleep onset insomnia (binary outcome variable) and a number of factors. The goodness-of-fit was assessed with the Hosmer and Lemeshow's test. All statistical analyses were conducted using SPSS (V.11.5.1, SPSS Inc, Chicago, Ill).

\section{Results}

\section{Study population}

Questionnaires were completed on 109 children, 95 (87.2\%) of whom were boys (mean age $( \pm \mathrm{SD}) 10.5 \pm 3.0 \mathrm{yrs})$ and $14(12.8 \%)$ were girls $(10.7 \pm 2.6 \mathrm{yrs}$ ) (Table 1). Some $88(80.7 \%)$ children used stimulants, $23(21.1 \%)$ used the following medication for sleep problems ( $\mathrm{n} ; \%)$ : tricyclic agents $(1 ; 0.9 \%)$, melatonin $(22$; $20.2 \%)$, and benzodiazepines $(1 ; 0.9 \%)$. The following psychiatric co-morbid disorders were diagnosed earlier by a physician or psychologist as was reported by the parents $(\mathrm{n} ; \%)$ : depressive disorder $(10 ; 9.2 \%)$, anxiety disorder $(11 ; 10.1 \%)$, 
Gilles de la Tourette/tic disorder (10;9.2\%), motor disorder $(29 ; 26.6 \%)$, mental disability $(1 ; 0.92 \%)$, auristic disorder/pervasive developmental disorder $(1 ; 0.92 \%)$, speech or language disorder $(8 ; 7.3 \%)$, and disruptive behavioral disorder (53; $48.6 \%$.

\section{Prevalence of sleep-related problems}

Table 2 shows the prevalence of sleep-related problems separately for children with versus without stimulant treatment. Sleep onset problems occurred "often" in $45.5 \%(40 / 88)$ of the children receiving stimulant treatment and in $28.6 \%(6 / 21)$ of the ADHD-children without use of stimulants (between-group difference; $p=0.008$ ). The between-group differences in other sleep-related problems were not significant (alpha was lowered from 0.05 to 0.00294 , Bonferroni correction).

Table 1. Demographic and clinical characteristics of children with diagnosed ADHD.

\begin{tabular}{l|l}
\hline & $n=109$ \\
\hline Age, years (mean \pm SD) & $10.6 \pm 2.8$ \\
Male, no. (\%) & $95(87.2)$ \\
Co-morbidity, no. (\%) & $10(9.2)$ \\
Depressive disorder & $11(10.1)$ \\
Anxiety disorder & $10(9.2)$ \\
Gilles de la Tourette/tic disorder & $29(26.6)$ \\
Motor disorder & $1(0.92)$ \\
Mental disability & $1(0.92)$ \\
Autistic disorder/pervasive developmental disorder & $8(7.3)$ \\
Speech/language disorder & $53(48.6)$ \\
Disruptive behavior disorder & \\
Medication use, no. (\%) & $88(80.7)$ \\
Stimulants & $1(0.9)$ \\
Tricyclic agents & $1(0.9)$ \\
Clonidin & $22(20.2)$ \\
Melatonin & $1(0.9)$ \\
Benzodiazepines &
\end{tabular}


Table 2. Prevalence of sleep-related problems in stimulant-medicated (ADHDmed) and unmedicated (ADHDnon) children with diagnosed ADHD.

\begin{tabular}{l|lll}
\hline & $\begin{array}{l}\text { ADHDmed } \\
\mathbf{( \% )}(\mathbf{n}=\mathbf{8 8})\end{array}$ & $\begin{array}{l}\text { ADHDnon } \\
(\%) \mathbf{n = 2 1 )}\end{array}$ & $\begin{array}{l}\boldsymbol{p} \text { group } \\
\text { difference }\end{array}$ \\
\hline Bedtime resistance & 37.1 & 25.0 & 0.50 \\
Difficulty falling asleep & 45.5 & 28.6 & $0.0080^{*}$ \\
Afraid of sleeping in dark & 8.9 & 5.0 & 0.84 \\
Leaves bedroom & 25.8 & 10.0 & 0.20 \\
Bedwetting & 9.0 & 10.5 & 0.97 \\
Talks during sleep & 11.1 & 25.0 & 0.26 \\
Teeth grinding & 7.8 & 15.0 & 0.60 \\
Snores & 4.5 & 5.0 & 0.99 \\
Awakens during night & 10.1 & 15.8 & 0.76 \\
Nightmares & 5.6 & 5.0 & 0.062 \\
Enters parental bedroom during night & 7.9 & 10.0 & 0.95 \\
Restless sleep & 30.3 & 15.0 & 0.096 \\
Bangs head/rocks & 4.4 & - & 0.38 \\
Difficulty waking up & 12.2 & 5.0 & 0.51 \\
Wakes up too early & 16.7 & 25.0 & 0.68 \\
Awakens in a bad mood & 10.0 & 20.0 & 0.37 \\
Falls asleep during daytime & 1.1 & - & 0.84 \\
\hline$*$ Signicant difference, $p \leq 05$, double & & & \\
\hline
\end{tabular}

* Significant difference, $p \leq 0.05$, double sided.

Sleep-wake characteristics in children with ADHD with versus without sleep onset insomia The sample was divided into two groups: $46(42.2 \%)$ children with ADHD and sleep onset insomnia (i.e. parents reported that the child "often" suffered from sleep onset problems) (ADHD-SOI), and $63(57.8 \%)$ children with ADHD but without sleep onset insomnia (i.e. parents reported "occasional" or "no" sleep onset problems) (ADHD-noSOI). Mean estimated sleep onset ( \pm SD) was 22:00 \pm 1:03 $\mathrm{h}$ in ADHD-SOI and 20:43 \pm 1:04 $\mathrm{h}$ in ADHD-noSOI (between-group; $p<0.001$ ) (Table 3). Sleep latency was $1: 22 \pm 0: 42 \mathrm{~h}$ in ADHD-SOI and 0:25 $\pm 0: 17$ $h$ in ADHD-noSOI ( $p<0.001)$. The difference in bedtime between ADHD-SOI $(20: 41 \pm 0: 56 \mathrm{~h})$ and ADHD-noSOI $(20: 28 \pm 1: 08 \mathrm{~h})$ was not significant $(p=0.29)$. There was a significant difference $(p<0.001)$ in total sleep duration between ADHD-SOI $(9: 11 \pm 1: 03 \mathrm{~h})$ and $\operatorname{ADHD}$-noSOI $(10: 20 \pm 1: 06 \mathrm{~h})$. Furthermore, we 
found a small but significant difference $(p=0.02)$ in wake up time between ADHDSOI $(7: 12 \pm 0: 15 \mathrm{~h})$ and ADHD-noSOI $(7: 04 \pm 0: 16 \mathrm{~h})$. The presence of sleep onset insomnia was not significantly related to age $(\mathrm{R}=-0.07 ; p=0.47)$, or gender $\left(\chi^{2}=1.094 ; p=0.30\right)$.

Table 3. Retrospective subjective sleep characteristics of children with ADHD and parentally reported sleep onset insomnia (ADHD-SOI) and children with ADHD without insomnia (ADHD-noSOI).

\begin{tabular}{l|lll}
\hline & $\begin{array}{l}\text { ADHD-SOI } \\
(\mathrm{n}=46)\end{array}$ & $\begin{array}{l}\text { ADHD-noSOI } \\
(\mathrm{n}=63)\end{array}$ & $p(\mathrm{t})$ \\
\hline Age, years & $10.2 \pm 2.6$ & $10.7 \pm 3.2$ & 0.43 \\
Bedtime, h:min & $20: 41 \pm 0: 56$ & $20: 28 \pm 1: 08$ & 0.29 \\
Sleep latency, h:min & $1: 22 \pm 0: 42$ & $0: 25 \pm 0: 17$ & $<0.001^{*}$ \\
Sleep onset, h:min & $22: 00 \pm 1: 03$ & $20: 43 \pm 1: 04$ & $<0.001^{*}$ \\
Total sleep duration, h:min & $9: 11 \pm 1: 03$ & $10: 20 \pm 1: 06$ & $<0.001^{*}$ \\
Wake up time, h:min & $7: 12 \pm 0: 15$ & $7: 04 \pm 0: 16$ & $0.02^{*}$ \\
\hline
\end{tabular}

$\triangle$ Group means \pm standard deviations.

* Statistically significant difference between ADHD-SOI and ADHD-noSOI ( $p \leq 0.05$, two-sided).

\section{Other sleep-related problems}

Table 4 shows relationship of sleep onset insomnia with other sleep-related problems (statistical significance lowered from 0.05 to 0.003125 , Bonferroni correction). Sleep onset insomnia was significantly related to bedtime resistance $(p<0.0001)$, leaving bedroom $(p<0.0001)$, nightmares $(p=0.0025)$, and difficulty waking up $(p<0.0001)$. Furthermore, there was a trend towards significance for entering parental bedroom during night $(p=0.0042)$, awaking during the night $(p=0.0032)$, restless sleep $(p=0.0032)$, and being afraid of sleeping in the dark $(p=0.0050)$.

\section{Stimulant treatment}

Retrospective reports by parents showed that in 16 children $(19.5 \%)$ sleep onset problems started following commencement with stimulant therapy, in 12 children $(14.6 \%)$ existing sleep onset problems were exacerbated, in 52 children (63.4\%) sleep remained the same, and in two children $(2.42 \%)$ sleep onset insomnia improved after a start with stimulant therapy $\left(\chi^{2}=47.4 ; p<0.0001\right)$ (Figure 1$)$. 


\section{Co-morbid psycbiatric disorders}

Depressive disorder was significantly related to insomnia $\left(\chi^{2}=10.46 ; p=0.005\right)$, while the relationship of insomnia with other psychiatric co-morbidities did not reach significance (level of significance was lowered from 0.05 to 0.00625 , Bonferroni correction).

Table 4. Relationship of parentally reported sleep onset insomnia with other sleep-related problems.

\begin{tabular}{l|ll}
\hline & Chi square & $p$ (chi square) \\
\hline Bedtime resistance & 26.33 & $<0.0001^{*}$ \\
Afraid of sleeping in dark & 10.55 & 0.0050 \\
Leaves bedroom & 25.97 & $<0.0001^{*}$ \\
Bedwetting & 0.96 & 0.62 \\
Talks during sleep & 2.81 & 0.25 \\
Teeth grinding & 9.10 & 0.011 \\
Snores & 7.46 & 0.024 \\
Awakens during night & 11.47 & 0.0032 \\
Nightmares & 11.96 & $0.0025^{*}$ \\
Enters parental bedroom during night & 10.95 & 0.0042 \\
Restless sleep & 11.50 & 0.0032 \\
Bangs head/rocks & 6.14 & 0.046 \\
Difficulty waking up & 21.89 & $<0.0001^{*}$ \\
Wakes up too early & 0.85 & 0.71 \\
Awakens in a bad mood & 4.82 & 0.090 \\
Falls asleep during daytime & 1.23 & 0.54 \\
\hline
\end{tabular}

* Statistically significant (set at $p<0.003125$, double sided, Bonferroni correction).

Family bistory of sleep onset insomnia

Family history of childhood sleep onset insomnia was $41.3 \%$ in ADHD-SOI ( $\mathrm{m}$ (other) $=10 ; \mathrm{f}$ (ather) $=4$; $\mathrm{s}$ (sibling $)=5)$, while $23.8 \%$ in $\mathrm{AHDH}$-noSOI $(\mathrm{m}=6 ; \mathrm{f}=3$; $\mathrm{s}=6$; missing=1), $\left(\chi^{2}=3.98 ; p=0.046\right),(\mathrm{OR}=2.33,95 \%$ CI 1.03-5.52).

Key factors associated with insomnia

A step-forward logistic regression analysis, with stimulant use, presence of familial childhood insomnia, depressive disorder, nightmares, and bedtime resistance 
introduced as independent variables, revealed a significant contribution of the following factors: stimulant use $(p=0.050)$, depressive disorder $(p=0.029)$, and bedtime resistance $(p<0.001)$. Presence of familial childhood insomnia or nightmares did not add significantly to the model. Further analyses (Hosmer and Lemeshow test) showed that the observed and predicted probabilities were not significantly different from each other $\left(\chi^{2}=0.46 ; p=0.93\right)$, which indicated that the model satisfactorily fitted the data. The percentage of individuals correctly predicted by this model as having sleep onset insomnia was $77.8 \%$, with a sensitivity of $64 \%(95 \%$, CI $49-78 \%)$, a specificity of $78 \%(95 \%$, CI $76-94 \%)$, and a likelihood ratio of 5.1 .

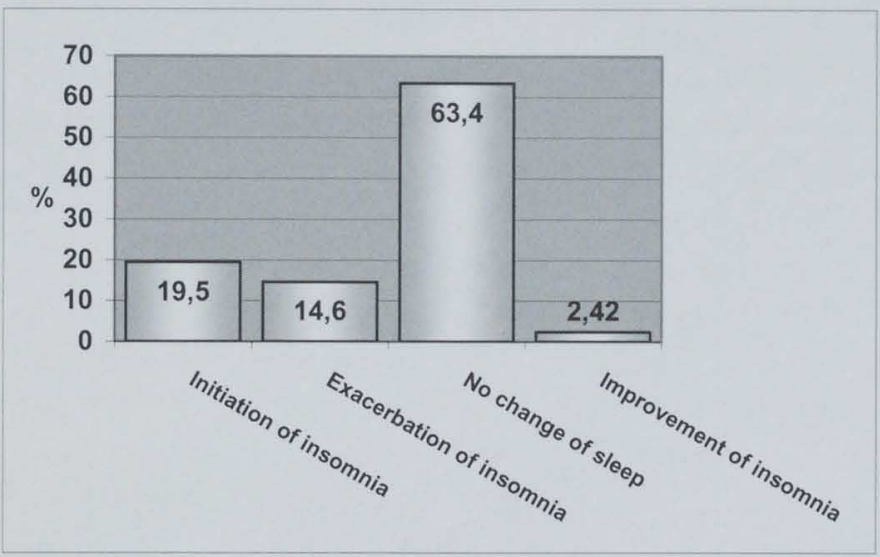

Figure 1. The effect of starting stimulant treatment on sleep in children with ADHD.

\section{Discussion}

The present results showed that stimulant use, presence of depressive disorder, and bedtime resistance were the major factors related to sleep onset insomnia in an outpatient population of children with diagnosed ADHD. The retrospective subjective sleep characteristics indicated that the complaints of insomnia were indeed accompanied with a longer sleep latency, later sleep onset, and a shorter total sleep duration.

The high prevalence of sleep onset insomnia of $45.5 \%$ found in our stimulant treated children with ADHD corresponds with high rates of $36.4-61.3 \%$ found previously [50;193], and far exceeds the prevalence of $28.6 \%$ that we found in 
children with ADHD without stimulant treatment. The detrimental effect of stimulants on sleep was further corroborated by our finding that a substantial number of children developed sleep onset insomnia following a start with stimulant treatment. Two possible mechanisms may have contributed to the negative effect of stimulants on sleep: 1) through enhancement of the neurotransmission of catecholamines (norepinephrine and dopamine) leading to increased sympathetic activity; 2) due to a rebound effect of irritability and hyperactivity taking place when the medication wears off.

Depressive disorder was the second major factor related to insomnia, which is in line with previous studies in adults and adolescents (without ADHD) [133;161]. An explanation may be that rumination, negative thoughts and worry were associated with increased physical and mental arousal, which are generally not compatible with sleep onset [59]. However, it needs to be recognized that the cross-sectional retrospective design of our study does not allow for an inference of causality. It remains unknown whether in children with ADHD insomnia was a precursor, symptom, residual symptom, or a side effect of the depression [109]. Namely, recent findings in adults suggested that insomnia can be a precursor or risk factor to the onset of major depressive disorder [73]. Of note, depressive disorder was found strongly associated to ADHD, which may be intermediated by the presence of insomnia [32]. One earlier study in children with ADHD found no relationship between depressive disorder and insomnia [137], however, in this study $28 \%$ of the children with ADHD used tricyclic antidepressants, which might have alleviated the depressive disorder and, consequently, may have masked its effect on sleep.

The third main factor related to insomnia was bedtime resistance. Possibly, bedtime resistance was induced by the aversive prospect of not being able to sleep and lying in bed awake. However, in some cases frequent struggles and arguments with parents in the evening might have caused sleep-disturbing cognitive or physiological hyperarousal, although we did not find a relationship of insomnia with disruptive behavior disorder. Another explanation may be that parents of children with ADHD and sleep onset insomnia might have 'over-reported' behavioral problems due to a perceptual bias. Further research is needed to clarify the nature and direction of the relationship between bedtime resistance and sleep onset insomnia.

There are several limitations to this study that need to be considered. First, our sample consisted of children referred to pediatric clinics, which suggests that the results may not pertain to other populations or settings. Nevertheless, the $28.6 \%$ prevalence of sleep-related problems that we found in medication-free children corresponded well with $27.8 \%$ and $32.1 \%$ found previously in psychiatric and 
community ADHD samples [50;193]. Recent sleep studies in the general child population showed prevalence rates of sleep onset insomnia of 8 to $15 \%$ [27;135;156], which are markedly lower than that found in the current study. Although the introduction of other clinical or normal comparison groups might have led to more information regarding the generalizability of the present results, we chose to limit this study to the ADHD population since our main interest was to identify the main factors associated with sleep onset insomnia within this group.

A recent study in insomniac adults using a semi structured clinical interview revealed that insomnia was strongly related to stress at work-school, family issues, and health problems [23]. We did not measure such psychological or systemic factors since these are usually identified by clinical interviews and are hard to investigate accurately by means of parental questionnaires. Furthermore, we did not study the potential role of sleep-disturbing syndromes such as restless legs syndrome, period limb movement disorder, or sleep-disordered breathing, since the diagnosis of these disorders requires extensive diagnostic testing. Inadequate sleep hygiene often plays an important preceding or perpetuating role in childhood insomnia, however, recent findings indicated that this factor is not related to chronic sleep onset insomnia in children with ADHD [212b].

Another methodological issue is that we did not perform diagnostic procedures for co-morbid psychiatric disorders, but alternatively asked the parents whether these disorders were officially diagnosed by a physician or psychologist. Notwithstanding, the psychiatric co-morbidity rates in our study were somewhat lower, but comparable, to those found in previous ADHD studies [1;32].

A last issue we want to highlight is that sleep was estimated retrospectively and subjectively. Studies in children with ADHD have shown low concordance between parent reports of sleep problems and objective measures [52;211]. The fact that the subjective sleep characteristics in the current group of children with ADHD and insomnia were typical of insomniacs, was supportive of the presence of sleep onset problems but may as well have been caused by biases or differences in parental perception. Furthermore, the association found between stimulant use and insomnia problems may partly be ascribed to the fact that parents may have been aware of the widely assumed sleep-related side effects of stimulants.

To summarize, this study shows that parents of children with a diagnosis of ADHD and receiving stimulant medication, with an additional diagnosis of depression, and described by their parents as difficult to get to bed describe high levels of initial sleep insomnia in these children. However, we emphasize that, considering the above limitations, the present findings should be treated with care and replication in future studies is necessary. 


\section{4}

Sleep hygiene and actigraphically evaluated sleep characteristics in children with attention-deficit/ hyperactivity disorder and chronic sleep onset insomnia

Van der Heijden, K.B., Smits, M.G., Gunning, W.B. Sleep hygiene and actigraphically evaluated sleep characteristics in children with attention-deficit/ hyperactivity disorder and chronic sleep onset insomnia. J Sleep Res., 2006, 15: 53-60. 


\begin{abstract}
In the present study we investigated sleep hygiene and actigraphically evaluated sleep in 74 medication naive children, aged 6-12 yrs, with rigorously diagnosed ADHD and chronic sleep onset insomnia (ADHDSOI), and 23 ADHD controls without insomnia (ADHD-noSOI). Between-group differences were analyzed of lights out (sleep log), actigraphically evaluated sleep onset, - latency, total sleep duration, actual sleep time; and sleep hygiene as measured with the Children's Sleep Hygiene Scale. We found a significant difference $(p<0.001)$ in mean $( \pm \mathrm{SD})$ sleep onset between the ADHD-SOI group (21:49 $\pm 0: 56 \mathrm{~h})$ and ADHDnoSOI group $(20: 41 \pm 0: 45 \mathrm{~h})$. Sleep latency was significantly $(p<0.001)$ longer in ADHD-SOI $(0: 53 \pm 0: 25 \mathrm{~h})$ as compared to ADHD-noSOI $(0: 26$ $\pm 0: 25 \mathrm{~h}$ ). The difference in total sleep duration between ADHD-SOI (9:42 $\pm 0: 44 \mathrm{~h}$ ) and ADHD-noSOI (10:09 $\pm 0: 43 \mathrm{~h}$ ) was not significantly different $(p=0.18)$. The group difference in actual sleep time was also not significant (8:43 $\pm 0: 52 \mathrm{~h}$ in ADHD-SOI vs. 9:13 $\pm 1: 16 \mathrm{~h} ; p=0.40)$. There was no significant difference $(p=0.17)$ in mean $( \pm$ SD) total sleep hygiene score between the ADHD-SOI group $(56.4 \pm 10.5)$ and ADHD-noSOI group (53.0 \pm 10.6$)$. We conclude that there were differences in sleep onset and sleep latency between children with ADHD and chronic sleep onset insomnia and those without insomnia, however, sleep hygiene practices were similar and did not relate to sleep characteristics.
\end{abstract}




\section{Introduction}

Chronic sleep onset insomnia occurs often in children with attentiondeficit/hyperactivity disorder (ADHD) [52;137;148;149;154;189] with reported rates of up to $28 \%$ in medication free children with ADHD [50], against $8-15 \%$ in the normal child population [27;135;156]. Childhood insomnia is a serious and severe disorder with a wide-ranging impact on the child's health, mood, behavior, and cognition $[58 ; 205 ; 231]$. Its daytime sequelae can be very similar to symptoms of ADHD and may therefore aggravate or even mimic ADHD [211].

We recently showed that chronic sleep onset insomnia in children with ADHD is associated with a delayed dim light melatonin onset and sleep-wake thythm, while sleep maintenance was normal [213]. These typical characteristics of a circadian rhythm sleep disorder point towards an involvement of a disturbed circadian pacemaker, possibly due to clock gene disturbances [16;68], however, the exact underpinnings are yet unknown.

Since some behavioral aspects of ADHD such as hyperactivity and disorganized characteristics might be of influence on the ability to fall asleep, we wanted to know the relationship of chronic sleep onset insomnia with sleep hygiene, which is defined as all behavioral and environmental factors that precede sleep and may interfere with sleep. It has been shown that inadequate sleep hygiene can deteriorate sleep [30] and sleep hygiene improving measures can increase sleep quality [95;115].

In the present study, we compared sleep hygiene in children with ADHD and chronic sleep onset insomnia (SOI) to that in children with ADHD without sleep complaints. Our hypothesis was that children with ADHD and SOI show poorer sleep hygiene measures as compared their counterparts without insomnia. None of the children in the study used psychotropic medication such as methylphenidate, clonidin, and benzodiazepines to exclude a possible influence of these medicines on sleep. All children were subjected to a rigorous diagnostic evaluation of ADHD and chronic SOI to increase homogeneity of the population. Since ADHD exists in different subtypes, i.e. ADHD-inattentive, ADHD-hyperactive/impulsive, and ADHD-combined subtype, and often manifests with disruptive behavior disorder, we also examined the relationship between sleep hygiene and these factors.

\section{Methods}

\section{Subjects}

Between November 2001 and April 2004, 117 children (aged 6-12 yrs) were referred by seven community mental health institutions and three pediatric departments of non-academic hospitals in the Netherlands to an academic child 
psychiatry outpatient clinic (former affiliation of $\mathrm{KH}$ ) for examination of ADHD. Children diagnosed with ADHD and sleep onset insomnia were offered to participate in a study on the efficacy of melatonin treatment. Children with ADHD without insomnia were eligible to participate in a study on the effect of methylphenidate on sleep. A further 21 children (aged 6-12 yrs) were recruited through advertisements in magazines for participation in one of the aforementioned studies. A total of 138 children took part in the current study.

The studies were approved by the Central Committee on Research Involving Human Subjects (CCMO), conducted according to the European Guidelines for Good Clinical Research Practice in children (Committee for Proprietary Medicinal Products, 1997), and followed the 1983 revised provisions of the 1975 Declaration of Helsinki. Informed consent was obtained from the participating children's parents.

Exclusion criteria were: total IQ less than 80 , pervasive developmental disorder, chronic pain, or used stimulants, melatonin, neuroleptics, benzodiazepines, clonidin, antidepressants, hypnotics, or beta blockers within four weeks before enrolment.

\section{Diagnosis}

Rigorous clinical diagnostic assessments were conducted on all children based on the Diagnostic and statistical manual of mental disorders, 4th edition (DSM-IV) [13]. The following ADHD subtypes were determined using DSM-IV: 1) predominantly inattentive type (ADHD-I) assigned to children who displayed more than six inattentive symptoms, but less than six hyperactive/impulsive symptoms; 2) predominantly hyperactive/impulsive type (ADHD-HI) when children displayed more than six hyperactive/impulsive symptoms but less than six inattentive symptoms; and 3) combined type (ADHD-C) for children with more than six symptoms of each set of symptoms.

Diagnostic procedures included clinical history, Diagnostic Interview Schedule for Children-Parent form (DISC-P), and the following behavioral questionnaires: Child Behavior Checklist [6], and Teacher's Report Form [5]. The criterion validity of the ADHD section of the DISC-P has recently been shown to be satisfactory [134]. Diagnostic procedures were carried out by a clinical psychologist $(\mathrm{KH})$ and board-certified child and adolescent psychiatrist (BG). Additionally, the Wechsler Intelligence Scale for Children, 3rd. ed. [228] short form (Block Design, Vocabulary) was administered (duration \pm 30 minutes) when IQ had not been assessed formetly and school performance had been poor in the last three years. 
The definition of SOI used in the current study was one that has been used in other studies [190;191;212] and its criteria received extensive discussion [214]. Children needed to meet all of the following criteria: 1) complaints of sleep onset problems by parents and/or child 2) sleep onset later than 20:30 h for children at age six years, and for older children 15 minutes later per year 3) sleep latency exceeding 30 minutes 4) occurrence on at least four weekdays per week, for longer than one year. The diagnosis was based upon a thorough patient history, a oneweek 24-hour actigraphy measurement, and a sleep hygiene questionnaire [84] (see below).

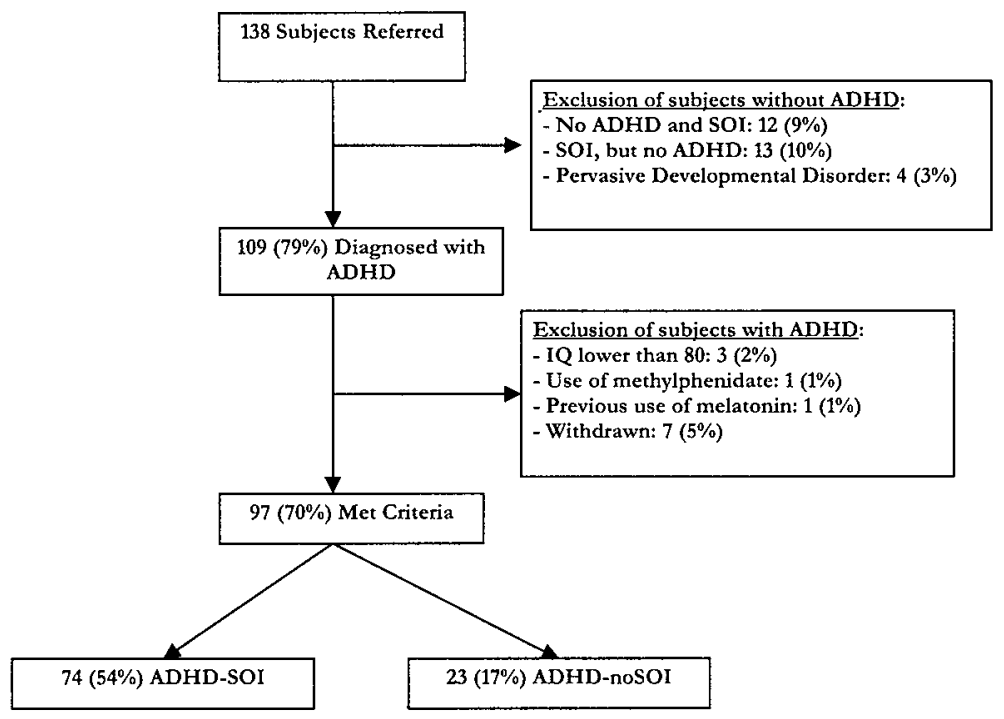

Figure 1. Trial profile. Children with ADHD and sleep onset insomnia (ADHD-SOI) and children with ADHD without insomnia (ADHD-noSOI).

Furthermore, the Dutch sleep disorders questionnaire (SDQ; Centre for Sleep and Wake Disorders, Westeinde Hospital, The Hague, The Netherlands) was used to evaluate periodic limb movements disorder/restless legs syndrome (PLMS/RLS) and sleep apnea syndrome (OSAS). The SDQ is the Dutch translation and adaptation of the Sleep Disorders Questionnaire developed by Douglass et al. [66] 
and was proved to be sufficiently reliable and valid [199]. The SDQ is a self-report questionnaire but was, in the present study, completed by parents and, where possible, in conjunction with the child. It encompasses 57 questions and has a fivepoint response scale $(1=$ never, $2=$ seldom, $3=$ sometimes, $4=$ often, and $5=$ always $)$.

\section{Procedures}

Sleep was evaluated using actigraphy (Actiwatch, Cambridge Neurotechnology Ltd, Cambridge, UK) worn on the non-dominant wrist 24 hours a day, for seven consecutive days. Actigraphy is a well validated instrument to evaluate sleep and circadian rhythm patterns in children [125]. Recordings of the amount of movement were made at one-minute epochs. A sleep log was completed by parents to mark lights out (when the child lies in bed, lights are dimmed and the child is expected to go to sleep) and get up time (time of leaving bed), and to provide supplemental data for identifying and rejecting actigraphy inaccuracies for example due to not wearing the device. Sleep onset (SO) was defined as the start of a period of at least 10 minutes of consecutively recorded immobile data following lights out; sleep latency (SL) defined as the time from lights out until sleep onset; total sleep duration (TSD) defined as the duration from sleep onset until awake time; and actual sleep time (AST) as the actual amount of sleep, calculated as TSD minus estimated time awake in the period from sleep onset until awake time. These sleep parameters were estimated from actigraphy data by a validated automatic scoring algorithm [113] (Actiwatch, Cambridge Neurotechnology Ltd, Cambridge, UK), which compares favorable to other such packages [38], and subsequent manual verification.

Sleep hygiene was assessed using the Children's Sleep Hygiene Scale (CSHS) [84]. The CSHS comprises of 25 short questions about sleep hygiene with a six-point response scale $(1=$ never; $6=$ always $)$ to be completed by the parents. The scale has shown adequate internal consistency (Cronbach's alpha; $\alpha=0.76$ ). The Adolescent Sleep Hygiene Scale was modified from the CSHS and has been proved valid and reliable [115;116]. The CSHS was developed by the Sleep Research Laboratory of the University of Southern Mississippi and was translated into Dutch and backtranslated to English by a certified translation centre (Dutch version, Appendix II).

\section{Statistics}

Reverse-coded items of the CSHS were recoded to align all responses in a positive direction (high score means poor sleep hygiene), and mean total sleep hygiene score was calculated for children with ADHD and chronic sleep onset insomnia 
(ADHD-SOI) and children with ADHD without sleep onset problems (ADHDnoSOI). For each subject, sleep parameters were averaged over seven days.

Between group differences (ADHD-SOI vs. ADHD-noSOI) in sleep parameters and TSH were explored with general linear model univariate analysis of variance with group as fixed factor and age as a covariate, or with Mann-Whitney $U$ test when data were found not to be normally distributed. Significance level was set at $p<0.05$, two-tailed. Between-group differences in item scores were tested with Mann-Whitney $U$, with adjusted significance level $(\phi<0.01$, two-tailed) to account for multiple comparisons.

Relationship between TSH and sleep parameters was analyzed for the total group and each group separately with linear regression analysis. Furthermore, the influence of gender, age, comorbid disruptive behavior disorder, ADHD subtypes (ADHD-C, ADHD-I, and ADHD-HI, recoded into dummy variables), and severity of ADHD (defined as the number of ADHD symptoms shown out of nine per set of symptoms (i.e. ADHD-I and ADHD-HI)) on TSH was analyzed with linear regression analysis, or with Spearman's correlation for ordinal data. Betweengroup (ADHD-SOI vs. ADHD-noSOI) differences in severity of ADHD, and in scores on the insomnia-, PLMS/RLS-, and OSAS scales of the SDQ were analyzed with Mann-Whitney U test. Significance level was set at $p<0.05$, two-sided.

\section{Results}

\section{Subject characteristics}

A number of 138 children was referred of which $29(22 \%)$ were not diagnosed with ADHD, three $(2 \%)$ had an IQ<80, one $(1 \%)$ used methylphenidate, and one $(1 \%)$ used melatonin previously (Fig. 1). Seven (5\%) children withdrew for a variety of reasons, e.g. including moving or necessity for an urgent start of therapy. The ADHD-SOI group consisted of 74 children (mean age 9.1 years \pm 2.1 (S.D.)) and the ADHD-noSOI group of 23 children (mean age $7.9 \pm 1.8$ (S.D.)) $(p=0.014$ ). Group characteristics regarding gender, age, ADHD-subtype, and co-morbidity are shown in Table 1.

There were no between-group differences in severity of inattentive symptoms (7.83 \pm 1.49 in ADHD-SOI vs. $7.33 \pm 1.50$ in ADHD-noSOI) $(p=0.15)$, or hyperactive/impulsive symptoms $(6.52 \pm 2.19$ in ADHD-SOI vs. $7.17 \pm 2.15$ in ADHD-noSOI) $(p=0.14)$. Scores on the insomnia scale of the SDQ were $2.65 \pm$ 0.53 in ADHD-SOI and $2.06 \pm 0.45$ in ADHD-noSOI $(p=0.0082)$. Between-group differences in mean PLMS/RLS scores (1.61 \pm 0.66 in ADHD-SOI vs. $1.41 \pm 0.90$ in ADHD-noSOI) or OSAS scores (1.25 \pm 0.33 in ADHD-SOI vs. $1.27 \pm 0.35$ in ADHD-noSOI) were not significant ( $p=0.082$ resp. $p=0.82$ ). 
Table 1. Demographic and clinical characteristics in children with ADHD and chronic sleep onset insomnia and children with ADHD without insomnia.

\begin{tabular}{|c|c|c|c|}
\hline & $\begin{array}{l}\text { ADHD-SOI } \\
(N=74)\end{array}$ & $\begin{array}{l}\text { ADHD-noSOI } \\
(\mathrm{N}=23)\end{array}$ & $\begin{array}{l}\text { Pooled } \\
(\mathrm{N}=97)\end{array}$ \\
\hline Age, years (mean $\pm S D)$ & $9.1 \pm 2.1$ & $7.9 \pm 1.8$ & $8.9 \pm 2.0$ \\
\hline Male, No. $(\%)$ & $56(76)$ & $20(87)$ & $76(78)$ \\
\hline \multicolumn{4}{|l|}{ Subtype, No. (\%) } \\
\hline ADHD-C & $56(75)$ & $19(83)$ & $75(76)$ \\
\hline ADHD-I & $15(20)$ & $3(13)$ & $18(19)$ \\
\hline ADHD-HI & $3(4)$ & $1(4)$ & $4(4)$ \\
\hline \multicolumn{4}{|l|}{ Comorbidity, No. $(\%)$} \\
\hline Disruptive behavioral disorder & $38(52)$ & $13(57)$ & $51(54)$ \\
\hline Anxiety disorder & $9(12)$ & $2(10)$ & $11(11)$ \\
\hline Affective disorder & $1(1)$ & $1(3)$ & $2(2)$ \\
\hline
\end{tabular}

ADHD-SOI: children with ADHD and chronic sleep onset insomnia; ADHD-noSOI: children with ADHD without insomnia; ADHD-C: ADHD-combined subtype; ADHD-I: ADHD inattentive subtype; ADHD-HI: ADHD hyperactive/impulsive subtype.

In ADHD-SOI there were four (5.4\%) children and in ADHD-noSOI one (4.3\%) with signs of PLMS/RLS as reported during the medical history taking. Signs of sleep apnea were present in one (4.3\%) child of the ADHD-noSOI group. Due to low frequency of occurrence and equal distribution over the two patient groups these children were not excluded.

\section{Actigraphy}

Mean $( \pm \mathrm{SD}$ ) sleep onset in the ADHD-SOI group was 21:49 $\pm 0: 56 \mathrm{~h}$ and in the ADHD-noSOI group 20:41 $\pm 0: 45 \mathrm{~h}(p<0.001)$ (Table 2$)$. Lights out was 20:57 \pm $0: 52 \mathrm{~h}$ in ADHD-SOI and 20:21 $\pm 0: 47 \mathrm{~h}$ in ADHD-noSOI $(p=0.08)$. There was a significant longer sleep latency in ADHD-SOI $(0: 53 \pm 0: 25 \mathrm{~h})$ as compared to ADHD-noSOI $(0: 26 \pm 0: 25 \mathrm{~h}),(p<0.001)$. The difference in total sleep duration between ADHD-SOI (9:42 \pm 0:44 h) and ADHD-noSOI (10:09 $\pm 0: 43 \mathrm{~h})$ was not significant $(p=0.18)$. The difference in actual sleep time between ADHD-SOI ( $8: 43$ $\pm 0: 52 \mathrm{~h})$ and $A D H D$-noSOI $(9: 13 \pm 1: 16 \mathrm{~h})$ was also not significant $(p=0.40)$. 


\section{Sleep bygiene}

Mean $( \pm \mathrm{SD})$ total sleep hygiene score in the ADHD-SOI group $(56.4 \pm 10.5)$ and ADHD-noSOI group $(53.0 \pm 10.6)$ did not differ significantly from each other $(p=0.17$ ) (Table 3). The ADHD-SOI group showed a significantly lower score on the first item (i.e. taking naps during the four hours before bedtime more often), $(p=0.011$ ), and a significantly higher score on item 7 (i.e. going to bed at about same time every evening less often), $(p=0.002)$, as compared to the ADHD-noSOI group. There was a significant relationship between total sleep hygiene score and lights-out $(\mathrm{R}=0.23 ; p=0.04)$ in the pooled group, however, not in the separate groups (Table 4).

Table 2. Sleep characteristics in children with ADHD and chronic sleep onset insomnia and children with ADHD without insomnia.

\begin{tabular}{llll}
\hline & $\begin{array}{l}\text { ADHD-SOI } \\
(\mathrm{n}=65)\end{array}$ & $\begin{array}{l}\text { ADHD-noSOI } \\
(\mathrm{n}=18)\end{array}$ & $p$ Value \\
\hline Lights out, h:min (mean $\pm \mathrm{SD})$ & $20: 57 \pm 0: 52$ & $20: 21 \pm 0: 47$ & 0.08 \\
Sleep onset, h:min (mean $\pm \mathrm{SD})$ & $21: 49 \pm 0: 56$ & $20: 41 \pm 0: 45$ & $<0.001^{*}$ \\
Sleep latency, h:min (mean $\pm \mathrm{SD})$ & $0: 53 \pm 0: 25$ & $0: 26 \pm 0: 25$ & $<0.001^{*}$ \\
Total sleep duration, h:min (mean $\pm \mathrm{SD})$ & $9: 42 \pm 0: 44$ & $10: 09 \pm 0: 43$ & 0.18 \\
Actual sleep time, h:min (mean $\pm \mathrm{SD})$ & $8: 43 \pm 0: 52$ & $9: 13 \pm 1: 16$ & 0.40 \\
\hline
\end{tabular}

Group means \pm standard deviations. ADHD-SOI: children with ADHD and chronic sleep onset insomnia; $\mathrm{ADHD}$-noSOI: children with $\mathrm{ADHD}$ without insomnia.

* Significant difference between ADHD-SOI and ADHD-noSOI after correction for age ( $p \leq 0.05$, two-sided).

Total sleep hygiene score was not significantly related to any other sleep parameter (i.e. sleep onset, sleep latency, total sleep duration, or actual sleep time) or to gender $(\mathrm{R}=0.015 ; p=0.88)$, age $(\mathrm{R}=0.13 ; p=0.20)$, comorbid distuptive behavior disorder $(\mathrm{R}=0.081 ; p=0.43)$, ADHD subtypes $\mathrm{ADHD}-\mathrm{C} \quad(\mathrm{R}=0.16$; $p=0.11)$, ADHD-I $(\mathrm{R}=0.097 ; p=0.34)$, or ADHD-HI $(\mathrm{R}=0.080 ; p=0.43)$, or to severity of inattentive ADHD symptoms $(\mathrm{R}=-0.007 ; p=0.95)$ or hyperactive/impulsive ADHD symptoms $(\mathrm{R}=0.19 ; p=0.092)$. 
Table 3. Sleep hygiene practices in children with ADHD and chronic sleep onset insomnia and children with ADHD without insomnia.

\begin{tabular}{|c|c|c|c|c|}
\hline \multicolumn{2}{|c|}{ Item } & \multirow{2}{*}{$\begin{array}{l}\text { Mean } \\
\text { ADHD- } \\
\text { SOI } \\
(\mathrm{n}=74)\end{array}$} & \multirow{2}{*}{$\begin{array}{l}\begin{array}{l}\text { Mean } \\
\text { ADHD- } \\
\text { noSOI } \\
(n=23)\end{array} \\
1.09\end{array}$} & \multirow{2}{*}{$\begin{array}{l}p \\
\text { Value } \\
0.011 *\end{array}$} \\
\hline 1 & Naps within 4 hours before bedtime & & & \\
\hline 2 & Caffeine within 4 hours before bedtime & 2.22 & 1.83 & 0.13 \\
\hline 3 & Does things that are relaxing before bedtime & 3.23 & 3.17 & 0.92 \\
\hline 4 & Drinks a lot of liquids before bedtime & 2.45 & 2.30 & 0.49 \\
\hline 5 & Plays rough before bedtime & 3.11 & 3.39 & 0.50 \\
\hline 6 & Does things that are alerting before bedtime & 3.64 & 3.35 & 0.47 \\
\hline 7 & Goes to bed at about the same time every day & 2.19 & 1.73 & $0.002 *$ \\
\hline 8 & Complains about being hungry at bedtime & 2.27 & 2.26 & 0.89 \\
\hline 9 & Does things in bed that keeps him/her awake & 3.35 & 3.09 & 0.43 \\
\hline 10 & Goes to bed in the same place & 1.53 & 1.64 & 0.55 \\
\hline 11 & Goes to bed feeling upset & 1.78 & 1.61 & 0.44 \\
\hline 12 & Goes to bed with worries & 2.21 & 2.00 & 0.35 \\
\hline 13 & Sleeps in a darkened room & 3.07 & 1.83 & 0.029 \\
\hline 14 & Sleeps in a room that is too hot or too cold & 1.49 & 1.78 & 0.14 \\
\hline 15 & Sleeps in a room where there are loud noises & 1.32 & 1.26 & 0.76 \\
\hline 16 & Sleeps in alone (in his/her own bed) & 1.72 & 1.43 & 0.60 \\
\hline 17 & Sleeps in a room that is "stuffy" & 1.47 & 1.35 & 0.23 \\
\hline 18 & Sleeps all or part of the night with someone else & 2.24 & 1.91 & 0.50 \\
\hline 19 & Sleeps in a bed that is comfortable & 1.12 & 1.09 & 0.75 \\
\hline 20 & Sleeps in a home where someone smokes & 2.62 & 3.13 & 0.24 \\
\hline 21 & Has a calming bedtime routine & 3.63 & 3.48 & 0.69 \\
\hline 22 & Uses bed for things other than sleep & 2.68 & 2.57 & 0.62 \\
\hline 23 & Put to bed after falling asleep & 1.18 & 1.52 & 0.19 \\
\hline 24 & Stays up past usual bedtime & 2.93 & 2.26 & 0.027 \\
\hline 25 & Gets out of bed about same time in morning & 2.70 & 2.04 & 0.023 \\
\hline \multicolumn{2}{|c|}{ Total (SD) } & $56.4(10.5)$ & $53.0(10.6)$ & 0.17 \\
\hline
\end{tabular}

ADHD-SOI: children with ADHD and chronic sleep onset insomnia; ADHD-noSOI: children with ADHD without chronic sleep onset insomnia.

- Higher score represents worse sleep hygiene.

* Significant difference between ADHD-SOI and ADHD-noSOI ( $p \leq 0.01$, two-sided). 
Table 4. Relationship between sleep hygiene and sleep parameters.

\begin{tabular}{lllllll}
\hline & \multicolumn{2}{l}{ ADHD-SOI } & \multicolumn{2}{l}{ ADHD-noSOI } & \multicolumn{2}{l}{ pooled groups } \\
\hline & $\mathrm{R}$ & $p$ Value & $\mathrm{R}$ & $p$ Value & $\mathrm{R}$ & $p$ Value \\
\hline Lights out & 0.16 & 0.95 & 0.24 & 0.57 & 0.23 & $0.04^{*}$ \\
Sleep onset & 0.076 & 0.77 & 0.18 & 0.14 & 0.19 & 0.092 \\
Sleep latency & 0.11 & 0.66 & 0.09 & 0.48 & 0.031 & 0.78 \\
Total sleep duration & 0.14 & 0.57 & 0.009 & 0.94 & 0.055 & 0.62 \\
Actual sleep time & 0.14 & 0.28 & 0.063 & 0.80 & 0.054 & 0.63 \\
\hline
\end{tabular}

ADHD-SOI: children with ADHD and chronic sleep onset insomnia; ADHD-noSOI: children with ADHD without chronic sleep onset insomnia.

* Significant relationship ( $p<0.05$, two-sided).

\section{Discussion}

The results of the present study showed that children with ADHD and chronic sleep onset insomnia did not differ in sleep hygiene from their counterparts without insomnia, although there were marked between-group differences in sleep onset and - latency. Furthermore, sleep hygiene was not related to sleep onset, latency, total sleep duration, or actual sleep time, while we did find a relationship with lights out. Therefore, the present results do not support our initial hypothesis that sleep hygiene could play a role in the etiology of insomnia in children with ADHD. This lack of association of insomnia and sleep hygiene level was also found in adult insomniacs (without ADHD) [39;86], but disagree with more recent data [102].

We found that children with ADHD and chronic SOI napped less frequently within four hours before bedtime than those without insomnia. Two explanations can be proposed: 1) the insomniacs were less tired in the evening due to an elevated cognitive and physiological arousal level in the evening, as has been found in adult insomniacs $[85 ; 218]$; 2) the children with ADHD and chronic SOI were more tired than their counterparts without SOI but, as a consequence of their sleepiness, expressed more hyperactive behavior [8] in the evening, which hampered their ability to fall asleep.

Possibly, a disturbance of the circadian pacemaker plays a role in the delayed sleep onset, as suggested by our recent finding of a markedly delayed onset of endogenous melatonin in children with ADHD and chronic SOI [213]. The children with ADHD and chronic SOI in the present study showed a significantly 
later sleep onset of more than one hour and a longer sleep latency of approximately half an hour as compared to the children with insomnia, while sleep duration was not significantly different. These are characteristics of a delayed sleep phase syndrome and corroborate our previous finding that chronic SOI in ADHD is a circadian thythm sleep disorder [213].

Recent data showed that a polymorphism of the PER3 gene of the circadian pacemaker, which has previously been associated with delayed sleep phase syndrome in adults $[16 ; 68]$, is not likely to be involved [210]. Yet, undiscovered clock genes [200], or other still unknown factors might also play a role. It was recently shown that the most commonly precipitating factor of insomnia in adolescents and adults was stress at school or work [23], which we did not measure. Further studies on the influence of school-related stress in ADHD-related chronic SOI are needed.

We investigated children with ADHD who were without psychotropic medication, thus, a possible influence of such on sleep onset can be excluded. Furthermore, we selected a group of consecutively referred subjects who were rigorously diagnosed for ADHD according to well-established criteria [13] and clinical practice guidelines [2;67], and for chronic SOI with strict and extensively discussed diagnostic criteria [214]. In none of the children, sleep hygiene-, or actigraphy measurements were preceded by behavioral sleep interventions to avoid possible manipulation of these factors in the insomnia group. Furthermore, a confounding influence of ADHD severity is unlikely since it was not related to sleep hygiene, nor were there differences in ADHD severity between the insomniacs and the children without insomnia.

Actigraphy is a well validated instrument to measure sleep in children [125]. Furthermore, the present scores on the Children's Sleep Hygiene Scale (56.4 vs. 53.0) agree satisfactorily with previous data in a general population of 246 children from the United States of America (total score $=57.2$; see [84]), which suggests that the sleep hygiene level in our children with ADHD was normal. We could not demonstrate a relationship between sleep hygiene and ADHD subtype or disruptive behavioral disorder, which makes a confounding involvement of these factors unlikely.

There were several limitations to this study that need to be acknowledged. Firstly, we evaluated sleep with actigraphy and not with gold standard polysomnography. Therefore, we could not objectively evaluate for sleep disorders such as sleep disordered breathing, periodic limb movement disorder, restless legs syndrome, and sleep architecture disorders that may relate to chronic sleep onset insomnia and might, therefore, have confounded our data. Nonetheless, we have limited the 
chance that such sleep disorders were present by careful history taking [9;72;197;198;224]. Furthermore, we found low scores and no group differences on various scales of the sleep disorders questionnaire, which reduces the chance that these disorders might have had a confounding influence. Of note, the SDQ has not been validated for the use in parents. However, the SDQ insomnia scale was significantly higher in the insomnia group as compared to the group without insomnia, which suggests at least a reasonable sensitivity on some scales. Our main reason to estimate sleep actigraphically and not polysomnographically is that actigraphy provides the possibility to assess sleep-wake rhythm over several consecutive days, in contrast to polysomnography. A single night polysomnography measure may fail to properly characterize the full extent of the sleep problem since insomnia typically varies in severity across nights [124].

A second methodological issue is that a selection bias might have occurred due to the fact that a subset of the participants was recruited for participation in an ongoing study on melatonin efficacy. Consequently, the occurrence of chronic sleep onset insomnia of 76 percent in our ADHD sample was markedly higher than in earlier studies [50;193]. Notwithstanding the high proportion of insomniacs in the present study, the composition of gender, ADHD subtypes, and co-morbid disruptive behavioral disorder resembles previous findings in ADHD [1]. Furthermore, these variables were not related to sleep hygiene in the present study. In none of the children behavioral sleep interventions were employed by health care professionals before participating in the present study. However, a number of parents that participated in the present study and in the ongoing study on melatonin efficacy reported that, before entolment, they had (vainly) tried several approaches to let their child fall asleep more easily. This might have improved sleep practices and, therefore, have influenced the present findings.

A further limitation in the present study is that the Children's Sleep Hygiene Scale was not validated in the Dutch child population, nor were there any Dutch normative data available. However, we don't expect significant intercultural differences in sleep hygiene within western countries since definitions of sleep hygiene across studies are very similar in their core factors [196].

To conclude, the present results show that sleep hygiene is not a likely etiological factor in ADHD-related chronic SOI. Nevertheless, for individual patients in clinical practice, an evaluation of sleep hygiene can still be valuable to identify possible poor sleep hygiene practices that could affect sleep onset. Furthermore, we underline that further research is needed to confirm and strengthen the present results. 



\section{5}

Idiopathic chronic sleep onset insomnia in attention-deficit/hyperactivity disorder: a circadian thythm sleep disorder

Van der Heijden, K.B., Smits, M.G., Van Someren, E.J.W., Gunning, W.B. Idiopathic chronic sleep onset insomnia in attention-deficit/hyperactivity disorder: a circadian thythm sleep disorder. Chronobiol Int., 2005, 22: 559-570. 


\begin{abstract}
To investigate whether ADHD-related sleep onset insomnia is a circadian thythm disorder, we compared actigraphic sleep estimates, the circadian rest-activity thythm, and dim light melatonin onset (DLMO) in children with ADHD having chronic idiopathic sleep onset insomnia (SOI) with that in children with ADHD without sleep problems. Participants were 87 psychotropic-medication naive children, aged 6-12 yrs, with rigorously diagnosed ADHD and SOI (ADHD-SOI) and 33 children with ADHD without SOI (ADHD-noSOI) referred from community mental health institutions and pediatric departments of non-academic hospitals in the Netherlands. Measurements were one-week $24 \mathrm{~h}$ actigraphy recordings and salivary DLMO. The mean sleep onset time of 21:38 $\pm 0: 54 \mathrm{~h}$ (SD) in ADHD-SOI, which was significantly $(p<0.001)$ later than that of $20: 49 \pm$ $0: 49 \mathrm{~h}$ in ADHD-noSOI. DLMO was significantly later in ADHD-SOI $(20: 32 \pm 0: 55 \mathrm{~h})$ as compared to ADHD-noSOI (19:47 $\pm 0: 49 \mathrm{~h})(p<0.001)$. Wake-up time in ADHD-SOI was later as compared to ADHD-noSOI $(p=0.002)$. There were no significant between-group differences in sleep maintenance, as estimated by number of wake bouts and activity level in the least active $5 \mathrm{~h}$ period, or inter- and intradaily rhythm variability. We conclude that children with ADHD and chronic idiopathic sleep onset insomnia show a delayed sleep phase and delayed DLMO as compared to children with ADHD without SOI.
\end{abstract}




\section{Introduction}

Sleep onset problems occur twice as much in attention-deficit/hyperactivity disorder (ADHD) as in clinical controls [50;193], with reported rates up to $28 \%$ in medication-free children [50]. The etiology of ADHD-related chronic sleep onset insomnia is unknown. In a series of studies on childhood chronic sleep onset insomnia, we found in many children with ADHD, characteristics of a delayed endogenous circadian pacemaker, such as a delayed sleep onset, dim light melatonin onset (DLMO), and wake-up time [190;191].

The endogenous circadian pacemaker is the hypothalamic suprachiasmatic nucleus which endogeneously generates a near $24 \mathrm{~h}$ rhythm, and entrains to lightdark input ascending from the retina. One of the resulting rhythms is that of the pineal hormone melatonin. DLMO, i.e., the evening rise of endogenous melatonin, is thought to be the most reliable phase marker of the pacemaker [108]. Patients with a delayed sleep phase syndrome, which is due to a disturbed endogenous circadian pacemaker [187], show a markedly delayed DLMO and a greater phase angle between DLMO and wake-up time $[157 ; 187 ; 206]$.

In order to investigate whether ADHD-related sleep onset insomnia is a circadian thythm disorder, we compared actigraphic estimates of sleep-wake rhythm and DLMO in children with ADHD and chronic sleep onset insomnia (SOI) with that of children with ADHD without sleep problems. We used rigorous criteria for the diagnosis of $\mathrm{ADHD}$ since its diagnosis is complicated by the lack of a specific test or marker, heterogeneity of its manifestation, and frequent association with other psychiatric and neurodevelopmental disorders. Furthermore, all children were psychotropic-medication naïve because many different psychotropic drugs, such as methylphenidate [7;22;193;195;202], SSRI's [74], and benzodiazepines [94] can influence sleep. Temperature is a 'Zeitgeber' [169] and is related to changes in activity or excitement. Therefore, we investigated a possible confounding influence of the predominantly hyperactive/impulse ADHD-subtype and co-morbid oppositional defiant disorder.

\section{Methods}

Subjects

A total of 176 children, aged 6-12 yrs, underwent diagnostic evaluation of ADHD between November 2001 and October 2004. Those who were diagnosed with ADHD and chronic SOI were eligible to participate in an ongoing randomized, placebo-controlled trial on the efficacy of melatonin treatment for chronic SOI in ADHD. Children without sleep problems who were diagnosed with ADHD were eligible to participate in a study on the effect of methylphenidate on sleep. Children 
were excluded if they had a total IQ of less than 80 , pervasive developmental disorder, chronic pain, or used stimulants, melatonin, neuroleptics, benzodiazepines, clonidin, antidepressants, hypnotics, or $\beta$-blockers within 1 month before participation. Some 152 children were referred by seven community mental health institutions and three pediatric departments of non-academic hospitals in the Netherlands, and 24 children were recruited through advertisements in magazines for participation in the previously mentioned melatonin trial. The studies were approved by the Central Committee on Research Involving Human Subjects (CCMO), conducted according to the European Guidelines for Good Clinical Research Practice in children (Committee for Proprietary Medicinal Products, 1997), and followed the 1983 revised provisions of the 1975 Declaration of Helsinki. Informed consent was obtained from the participating children's parents.

\section{Diagnostic measures}

Rigorous clinical diagnostic assessments were carried out by a clinical psychologist $(\mathrm{KH})$ and board-certified child and adolescent psychiatrist (BG). Diagnostic procedures included clinical history, Diagnostic Interview Schedule for ChildrenParent form (DISC-P), and Child Behavior Checklist [6] and Teacher's Report Form [5]. Diagnosis of ADHD and other axis-I disorders was made based on the Diagnostic and Statistical Manual of Mental Disorders, 4th edition [13]. The criterion validity of the ADHD section of the DISC-P has recently been shown to be satisfactory [134]. Additionally, the WISC-III short form (Block Design, Vocabulary) was administered (duration $\pm 30 \mathrm{~min}$ ) when IQ had not been assessed formerly and school performance had been poor in the last three years.

The diagnosis of chronic sleep onset insomnia (SOI) was only made in children referred with chronic sleep onset problems and normal sleep maintenance. SOI was defined as in other studies [190;191]: 1) complaints of sleep onset problems by parents and/or child, 2) sleep onset later than 20:30 h for children at age $6 \mathrm{yrs}$, and for older children 15 mins later per year, 3) sleep latency exceeding $30 \mathrm{~min}$, and 4) presence of more than 4 days per week on average during at least more than one year. The sleep examination consisted of a rigorous clinical history, sleep diagnosis questionnaire [199] adapted for children, and sleep hygiene questionnaire [84]. In case of firm sleep hygiene inadequacies or other sleep-influencing factors, further examination or intervention was applied before enrolment. 


\section{Actigraphy}

Sleep was evaluated by wrist actigraphy (non-dominant arm) for $24 \mathrm{~h}$ per day during seven consecutive days. An actigraph (Actiwatch: Cambridge Neurotechnology, UK) is a well validated instrument to evaluate sleep and circadian rhythm patterns in children [125]. Recordings of the amount of movement were made at one-minute intervals. A sleep log was completed by parents to mark lights out (when the child lies in bed, lights are dimmed and the child is expected to go to sleep) and get-up time (time of leaving bed), and to provide supplemental data for identifying and rejecting actigraphy inaccuracies, for example, due to not wearing the device. Sleep onset $(\mathrm{SO})$ is defined as the start of a period of at least ten mins of consecutively recorded immobile data, following lights out; wake-up time (WT) is defined as the last epoch of immobility before the start of a ten-minute consecutive period of activity around the get-up time; sleep latency (SL) is defined as the time from lights out until sleep onset; total sleep duration (TSD) is defined as the duration from sleep onset until wake-up time; and wake bouts is defined as the actual number of episodes of activity from sleep onset until wake-up time. These sleep parameters were estimated from actigraphy data by a validated automatic scoring algorithm [113] (Actiwatch software, Cambridge Neurotechnology), which compares favorable to other such packages [38] and subsequent manual verification.

The following activity thythm parameters were calculated according to Van Someren, et al. [217]: Interdaily Stability (IS), defined as the degree of resemblance between the activity patterns on individual days, i.e., the invariability between the days; Intradaily Variability (IV), defined as the fragmentation of periods of rest (or sleep) and activity (or wakefulness), i.e., the frequency and extent of transitions between rest and activity; L5, defined as the average activity during the least active five-hour period in the average $24 \mathrm{~h}$ pattern. These variables are nonparametric in that they do not assume any specific waveform and, therefore, are more suitable for quantification of the highly non-sinusoidal activity thythm [217]. Actigraphy data were present in 66/87 of the ADHD-SOI group and 20/33 of the ADHD-noSOI group. Some data were missing due to technical problems.

\section{Dim light melatonin onset}

Assessment of endogenous melatonin occurred through collection of salivary samples hourly from 18:00-22:00 h (age 6-7 yrs) or 19:00-23:00 h (age 8-13 yrs) by chewing on a cotton plug (Salivetten: Sarstedt Nümbrecht, Germany) for one minute. To prevent suppression of melatonin secretion by bright light $[167 ; 236]$, curtains needed to be closed and only one dim light was allowed during the 
measurement period. DLMO, defined as the first time at which salivary melatonin reached a level of $4 \mathrm{pg} / \mathrm{mL}$, was calculated as the linearly interpolated time of the first sample with a level above $4 \mathrm{pg} / \mathrm{mL}$ that was preceded by a lower value [36;143]. Salivary melatonin concentrations were assayed by radioimmunoassay (RIA) as described previously [143]. DLMO data were present in $78 / 87$ of the ADHD-SOI group and 30/33 of the ADHD-noSOI group. It was not possible to determine the DLMO in all subjects because of an insufficient volume of collected saliva in some cases.

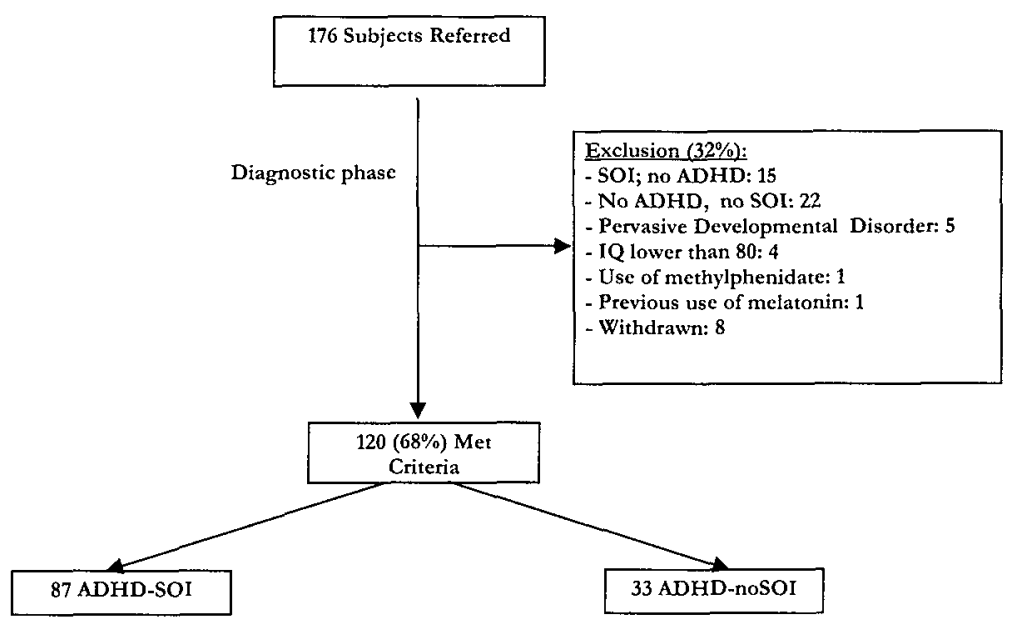

Figure 1. Trial profile. Children with ADHD and sleep onset insomnia (ADHD-SOI) and children with ADHD without sleep problems (ADHD-noSOI).

\section{Data analysis and statistics}

For each subject, sleep parameters were averaged over seven days, and the phase

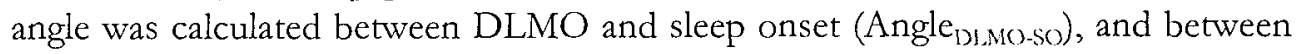

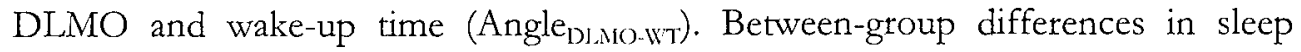
parameters, nonparametric circadian activity rhythm parameters, DLMO, and phase angles (Angle DIMO-SO $_{\text {O }}$ and Angle ${ }_{\mathrm{DIMO} \text { (WT }}$ ) were explored with GLM Univariate analysis of variance with group as a fixed factor and subsequently with age as a covariate. When data were found not to be normally distributed by the 
Kolmogorov-Smirnov test, group differences were assessed using the nonparametric Mann-Whitney test. Correction for inequality of variance, according to Levene's inequality of variance test, was employed when appropriate. Statistical significance was set at $p \leq 0.05$.

\section{Results}

Subjects

A total of 87 children were diagnosed with ADHD and SOI (ADHD-SOI), 33 with ADHD without sleep problems (ADHD-noSOI). Some 56 children did not meet the inclusion criteria and were excluded (Figure 1; trial profile). Group characteristics are shown in Table 1 . In 70\% of the cases the onset of SOI occurred before the age of three years, in $25 \%$ between the age of three to six yrs, and in $5 \%$ after the age of six yrs.

Table 1. Demographic and clinical characteristics of children with ADHD and sleep onset insomnia (ADHD-SOI) and children with ADHD without sleep onset insomnia (ADHD-noSOI).

\begin{tabular}{|c|c|c|}
\hline & ADHD-SOI $(n=87)$ & ADHD-noSOI $(n=33)$ \\
\hline Age in yrs, mean (SD) & $8.8(1.7)$ & $8.2(2.0)$ \\
\hline Male, No. $(\%)$ & $66(76)$ & $26(79)$ \\
\hline \multicolumn{3}{|l|}{ Comorbidity, No. $(\%)$} \\
\hline Oppositional defiant disorder & $50(58)$ & $21(65)$ \\
\hline Anxiety disorder & $10(12)$ & $3(10)$ \\
\hline Affective disorder & $1(1)$ & $1(3)$ \\
\hline \multicolumn{3}{|l|}{ ADHD-subtype, No. (\%) } \\
\hline ADHD-C & $69(79)$ & $25(77)$ \\
\hline ADHD-I & $15(17)$ & $5(16)$ \\
\hline ADHD-HI & $4(5)$ & $2(7)$ \\
\hline
\end{tabular}

ADHD-C: ADHD combined type; ADHD-I: ADHD predominantly inattentive type; ADHDHI: ADHD predominantly hyperactive/impulsive type.

\section{Actigrapby}

Children in the ADHD-SOI group showed a significantly $(p<0.001)$ later sleep onset of 21:38 $\pm 0: 54 \mathrm{~h}(\mathrm{SD})$ as compared to children in the ADHD-noSOI group 
$(20: 49 \pm 0: 49 \mathrm{~h}$ ) (Table 2). Lights-out did not differ significantly between the groups (20:47 $\pm 0: 52$ h vs. $20: 22 \pm 0: 48$ h; n.s.).

Table 2. Parameters of sleep, circadian activity rhythm, and DLMO in children with ADHD and chronic sleep onset insomnia (ADHD-SOI), and children with ADHD without sleep problems (ADHD-noSOI).

\begin{tabular}{|c|c|c|c|}
\hline & ADHD-SOI & ADHD-noSOI & $P(\mathrm{t})$ \\
\hline Lights-out & $\begin{array}{l}20: 47 \pm 0: 52 \\
(n=66)\end{array}$ & $\begin{array}{l}20: 22 \pm 0: 48 \\
(n=20)\end{array}$ & n.s. \\
\hline Slcep onset & $\begin{array}{l}21: 38 \pm 0: 54 \\
(n=66)\end{array}$ & $\begin{array}{l}20: 49 \pm 0: 49 \\
(n=20)\end{array}$ & $<0.001$ \\
\hline Sleep latency & $\begin{array}{l}0: 51 \pm 0: 25 \\
(n=66)\end{array}$ & $\begin{array}{l}0: 27 \pm 0: 26 \\
(n=20)\end{array}$ & $<0.001$ \\
\hline Wake-up time & $\begin{array}{l}7: 29 \pm 0: 39 \\
(n=66)\end{array}$ & $\begin{array}{l}6: 56 \pm 0: 46 \\
(n=20)\end{array}$ & 0.002 \\
\hline Get-up time & $\begin{array}{l}7: 44 \pm 0: 34 \\
(n=66)\end{array}$ & $\begin{array}{l}7: 16 \pm 0: 38 \\
(n=20)\end{array}$ & 0.003 \\
\hline Total sleep duration & $\begin{array}{l}9: 51 \pm 0: 41 \\
(n=66)\end{array}$ & $\begin{array}{l}10: 08 \pm 0: 44 \\
(n=20)\end{array}$ & n.s. \\
\hline Wake bouts, No. & $\begin{array}{l}34.8 \pm 18.1 \\
(n=66)\end{array}$ & $\begin{array}{l}34.2 \pm 24.7 \\
(n=20)\end{array}$ & n.s. \\
\hline DLMO & $\begin{array}{l}20: 32 \pm 0: 55 \\
(n=78)\end{array}$ & $\begin{array}{l}19: 47 \pm 0: 49 \\
(n=30)\end{array}$ & $<0.001$ \\
\hline Phase angle_DI.MO - Wake-up time & $\begin{array}{l}10: 55 \pm 0: 52 \\
(n=64)\end{array}$ & $\begin{array}{l}11: 09 \pm 0: 45 \\
(n=20)\end{array}$ & n.s. \\
\hline Phase angle_DLMO - Sleep onset & $\begin{array}{l}1: 06 \pm 0: 50 \\
(n=64)\end{array}$ & $\begin{array}{l}1: 02 \pm 0: 59 \\
(n=20)\end{array}$ & n.s. \\
\hline IS & $\begin{array}{l}0.63 \pm 0.15 \\
(n=66)\end{array}$ & $\begin{array}{l}0.63 \pm 0.31 \\
(n=20)\end{array}$ & n.s. \\
\hline IV & $\begin{array}{l}0.69 \pm 0.19 \\
(n=66)\end{array}$ & $\begin{array}{l}0.81 \pm 0.26 \\
(n=20)\end{array}$ & n.s. \\
\hline L5 & $\begin{array}{l}40.6 \pm 27.7 \\
(n=66)\end{array}$ & $\begin{array}{l}39.9 \pm 34.1 \\
(n=20)\end{array}$ & n.s. \\
\hline
\end{tabular}

DLMO: dim light melatonin onset; IS: interdaily stability; IV: intradaily variability; L5: average activity level in least active five-hour period. 
Sleep latency was significantly longer in children with ADHD and SOI $(0: 51 \pm$ $0: 25 \mathrm{~h})$ as compared to ADHD-children without SOI $(0: 27 \pm 0: 26 \mathrm{~h} ; p<0.001)$. Wake-up time was significantly later in the ADHD-SOI group $(7: 29 \pm 0: 39 \mathrm{~h})$ than in the ADHD-noSOI group (6:56 $\pm 0: 46 \mathrm{~h} ; p=0.002)$.
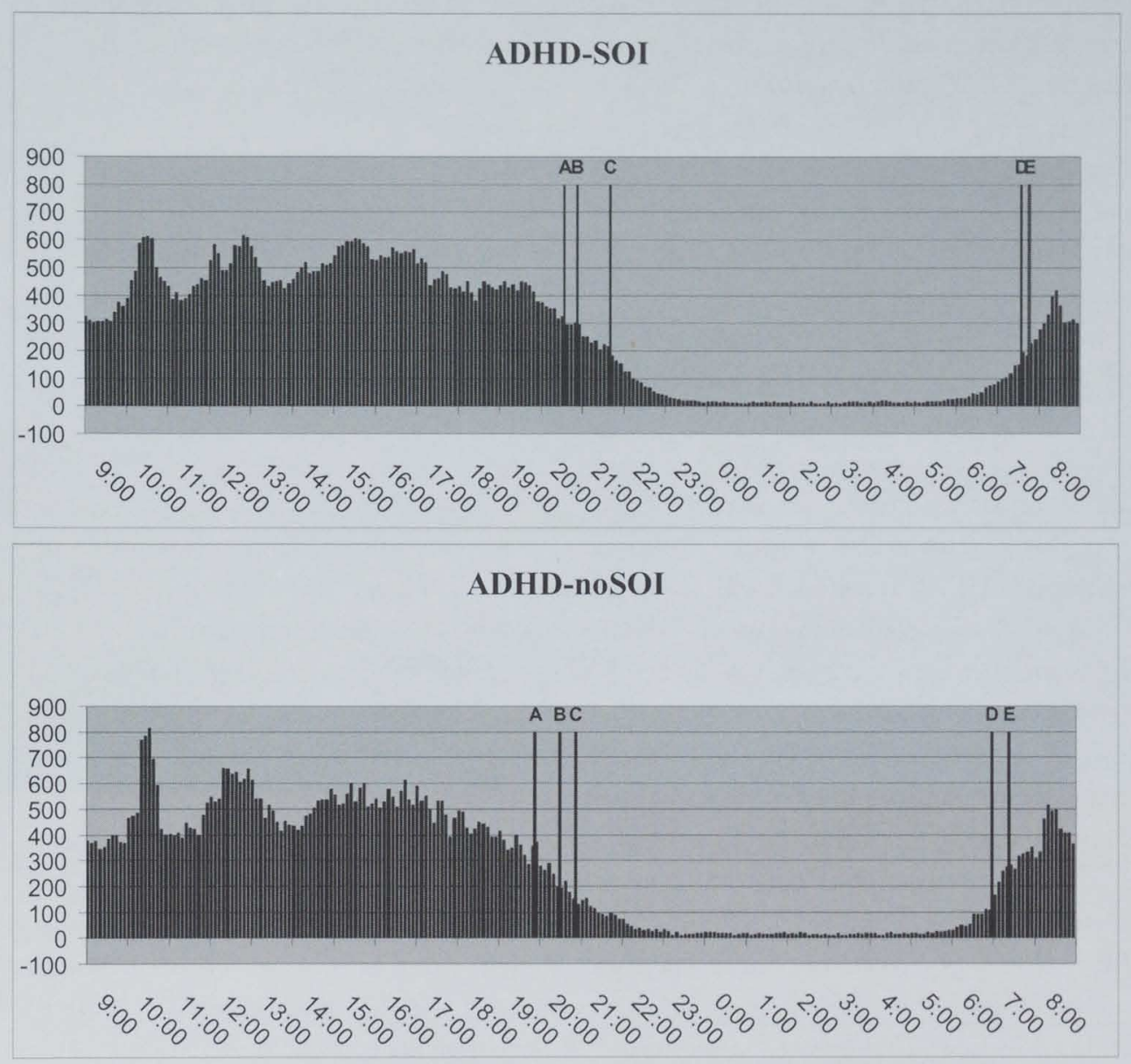

Figure 2. Average $24 \mathrm{~h}$ activity rhythm, with DLMO (A); lights-out (B); sleep onset (C); wake-up time (D); and get-up time (E) in children with ADHD and SOI (ADHD-SOI), and children with ADHD without sleep problems (ADHD-noSOI). The activity peaks reflect transport to school $( \pm 8: 30 \mathrm{~h})$, outside play or free play time $( \pm 10: 30 \mathrm{~h})$, and lunch break $( \pm 12: 30 \mathrm{~h})$. 
There was a significant difference in get-up time between the ADHD-SOI group $(7: 44 \pm 0: 34 \mathrm{~h})$ and ADHD-noSOI group $(7: 16 \pm 0: 38 \mathrm{~h} ; p=0.003)$. Total sleep duration in the ADHD-SOI group (9:51 $\pm 0: 41 \mathrm{~h}$ ) was 17 mins shorter compared to the ADHD-noSOI group (n.s.). The number of activity bouts in ADHD-SOI $(34.8 \pm 18.1)$ was not significantly different from that in ADHD-noSOI $(34.2 \pm$ 24.7 ; n.s.). The nonparametric circadian activity thythm variables of IS, IV, and L5 did not differ significantly between the groups. Significance of differences did not change after including age, ADHD-subtype, or presence of oppositional defiant disorder (ODD) as covariates.

\section{Dim light melatonin onset and phase angles}

DLMO was significantly later in the ADHD-SOI group $(20: 32 \pm 0: 55 \mathrm{~h})$ as compared to the ADHD-noSOI group (19:47 $\pm 0: 49 \mathrm{~h} ; p<0.001)$, also with age,

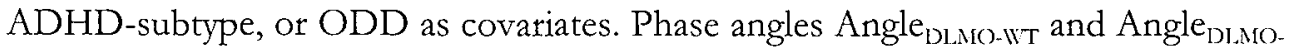
so were not significantly different. Figure 2 depicts for each group the $24 \mathrm{~h}$ activity rhythm averaged over 7 days, and average DLMO, lights-out, sleep onset, wake-up time, and get-up time.

\section{Discussion}

In children with ADHD and chronic sleep onset insomnia, sleep onset, wake-up time, and DLMO are delayed as compared to such of children with ADHD without sleep problems, while sleep maintenance remained normal. These characteristics are typical of a delayed sleep phase syndrome (DSPS) [142] and likely result from an endogenous circadian pacemaker fixed at a later phase than the desired sleep-wake schedule [232]. Hence, we suggest that idiopathic chronic sleep onset insomnia in children with ADHD is a circadian thythm sleep disorder. Nonetheless, we prefer to continue using the term "idiopathic sleep onset insomnia" tather than "delayed sleep phase syndrome", since the delayed sleep phase syndrome is still poorly defined and recognized in children [4;64].

We found no influence of behavioral factors on the SOI or DLMO, which suggests that behavioral factors did not play a role in the pathogenesis of SOI as Corkum et al. [52] suggested. Furthermore, use of psychotropic medication can be ruled out as an influencing factor, since all children were naive for this class of medication. There were no differences between groups in the time of lights-out, which indicates that the difference in sleep onset was not the result of differences in bedtimes. The possibility of a referral bias is small, since the study sample was a heterogeneous $\mathrm{ADHD}$ group referred from different care settings. Furthermore, the DLMO in the ADHD-noSOI group can be considered normal, since it differed 
only by 2 min from the previously published reference for a group of similar-aged control children without sleep problems [191].

Since we used well-validated and reliable measures of sleep and circadian thythm in a large group of rigorously diagnosed, psychotropic-medication naive children with $\mathrm{ADHD}$, we believe our findings are reliable and valid. However, several limitations may be taken into account. First, we did not perform objective diagnostic examination for other sleep disorders, such as sleep disordered breathing, periodic limb movement disorder, restless legs syndrome, and sleep architecture disorders, that may relate to chronic sleep onset insomnia and might, therefore, have confounded our data. Nevertheless, we have limited the chance that such sleep disorders were present by careful history taking [9;72;197;198;224].

We do not know the etiology of SOI in ADHD. It would be of interest to investigate the possibility of an increased risk of a PER3 clock gene polymorphism, which has previously been found in adults with the delayed sleep phase syndrome [16;68], which we are currently undertaking. In $70 \%$ of the children, the start of the sleep onset insomnia occurred before age three years, which suggests strong predisposing factors thereby reducing the chance that the sleep onset problems were precipitated by school, psychological, or physical problems [23]. A delayed DLMO predicts a greater efficacy of melatonin treatment of chronic childhood idiopathic insomnia [212], which is promising for the present children with ADHD and chronic sleep onset insomnia. Bright light therapy has been shown efficacious in adult delayed sleep phase syndrome [47;177]. We are currently conducting placebo-controlled, double-blind trials on the efficacy of melatonin treatment or bright light therapy in children with ADHD and chronic sleep onset insomnia. 

No evidence to support an association of PER3 clock gene polymorphism with attention-deficit/hyperactivity disorder related idiopathic chronic sleep onset insomnia

Van der Heijden, K.B., Blok, M.J., Spee, K., Archer, S.N., Smits, M.G., Curfs, L.M., Gunning, W.B. No evidence to support an association of PER3 clock gene polymorphism with attention-deficit/hyperactivity disorder related idiopathic chronic sleep onset insomnia. Biol Rhythm Res, 2005, 36: 381-388. 


\begin{abstract}
Idiopathic chronic sleep onset insomnia (SOI) in children with attentiondeficit/hyperactivity disorder (ADHD) shows typical characteristics of a delayed sleep phase syndrome and could therefore be considered a circadian rhythm sleep disorder. A variable number tandem repeat (VNTR) polymorphism of the clock gene PER3 is associated with the delayed sleep phase syndrome and, hence, may associate with ADHD-related chronic SOI as well. Here, we investigated an association between ADHD-related chronic SOI and the VNTR polymorphism of PER3 in 10 medication naive children with rigorously diagnosed ADHD and SOI (ADHD-SOI), and in 10 normal controls. Actigraphic sleep onset and sleep duration and salivary dim light melatonin onset (DLMO) were evaluated in ADHD-SOI. The 4repeat allele frequency was lower in ADHD-SOI (0.65) than in normal controls $(0.75)(\mathrm{p}=0.73)$ with an odds ratio of 0.62 (CI 0.16-2.4). In ADHD-SOI, mean $( \pm \mathrm{SD})$ DLMO $(21: 38 \pm 0: 50 \mathrm{~h})$, sleep onset $(22: 17 \pm$ $0: 46 \mathrm{~h})$, and sleep duration $(9: 26 \pm 0: 41 \mathrm{~h})$ were not significantly related to the 4-repeat allele frequency. The present findings suggest no association between ADHD-related idiopathic chronic sleep onset insomnia and the PER3 VNTR polymorphism.
\end{abstract}




\section{Introduction}

The human endogenous circadian pacemaker of the hypothalamus, the suprachiasmatic nucleus ( $\mathrm{SCN}$ ), has a regulating influence on sleep-wake thythm, and drives endocrinal and temperature rhythms. The SCN consists of a number of clock genes whose molecular products form interacting positive and negative autoregulatory feedback loops with a resulting near 24-hour cycle. The PER3 clock gene is part of the transcriptional inhibitory complex [118], and its variable number tandem repeat polymorphism (with 4 or 5 repeat units) has been associated with the delayed sleep phase syndrome (DSPS) $[16 ; 68 ; 160]$. In the northern hemisphere, the 4-repeat allele in PER3 showed a higher frequency in DSPS subjects when compared to the general population [16], while this frequency in DSPS was relatively lower in the southern hemisphere [160].

Chronic sleep onset insomnia (SOI) in children with ADHD closely resembles DSPS in that the sleep-wake thythm and the onset of endogenous melatonin production - the dim light melatonin onset (DLMO) - are markedly delayed, while sleep maintenance is unaffected [213]. The prevalence of chronic SOI is probably two to three times higher in children with ADHD (without psychotropic medication) than in the normal population [50;193], while its etiology is still unknown. We hypothesized that the 4-repeat allele of the PER3 clock gene is associated with ADHD-related chronic idiopathic sleep onset insomnia. Therefore, we investigated the PER3 VNTR polymorphism in a group of rigorously diagnosed children with ADHD with an early onset of SOI before age three years and a delayed value of DLMO and compared this to normal controls.

\section{Methods}

\section{Subjects}

Between November 2001 and July 2004, 176 children (6-12 years of age) were referted to our clinic by community mental health institutions and pediatric departments of non-academic hospitals in the Netherlands for examination of ADHD. The present study was part of a larger study on the efficacy of melatonin treatment which was approved by the Central Committee on Research Involving Human Subjects (CCMO), conducted according to the European Guidelines for Good Clinical Research Practice in children (Committee for Proprietary Medicinal Products, 1997), and followed the 1983 revised provisions of the 1975 Declaration of Helsinki. Informed consent was obtained from the participating children's parents.

Exclusion criteria were: total IQ $<80$, pervasive developmental disorder, used psychotropic medication that could influence sleep such as stimulants, melatonin, 
neuroleptics, benzodiazepines, clonidin, antidepressants, hypnotics, or $\beta$-blockers within one month before enrolment, and onset of SOI after age three years to reduce the chance that chronic sleep onset insomnia was the result of environmental factors (trauma, psychological problems etc.).

Controls were 10 randomly selected individuals who were referred to an academic clinical genetics department.

\section{Diagnosis}

Diagnosis of ADHD and other axis-I disorders was based on the Diagnostic and Statistical Manual of Mental Disorders, 4th edition, carried out by a clinical psychologist $(\mathrm{KH})$ and board-certified child and adolescent psychiatrist (BG) and included clinical history, Diagnostic Interview Schedule for Children-Parent form (DISC-P) [146], Child Behavior Checklist [6], and Teacher's Report Form [5]. SOI was defined as in other studies [190;191;212;214]: 1) complaints of sleep onset problems by parents and/or child 2) sleep onset later than 20:30 h for children at age six years, and for older children 15 minutes later per year 3) sleep latency exceeding 30 minutes 4) occurrence on at least four days per week, for longer than one year. The sleep examination consisted of a one-week, 24-hour actigraphy measurement, clinical history, sleep diagnosis questionnaire [199] adapted for children, and a sleep hygiene questionnaire [84]. Children were excluded when insomnia was due to firm sleep hygiene inadequacies or to other sleep-influencing factors such as restless legs syndrome (RLS) [9;197;224].

\section{Measurements}

Dim light melatonin onset: DNA was extracted from buccal cell samples from

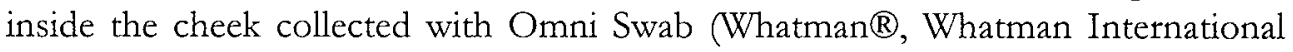
Ltd, Maidstone, UK). The PER3 length polymorphism was analyzed by PCR using the primers as described by Ebisawa et al. [68]. PCR product fragment lengths were analyzed by gel electrophoresis. Assessment of salivary endogenous melatonin and actigraphy occurred only in children with ADHD and chronic sleep onset insomnia because we expected only in this group a relation of these measures with the PER3 length polymorphism. Saliva samples were collected by chewing on a cotton plug (Salivetten ${ }^{\circledR}$, Sarstedt Nümbrecht, Germany) for one minute every hour from 18:00 to 22:00 h (age 6-7 years) or 19:00 to 23:00 h (age 8-13 years). Suppression of melatonin secretion by bright light $[167 ; 236]$ was prevented by closing of curtains and using not more than one dim light during the measurement period. Salivary melatonin concentrations were assayed with radioimmunoassay (RIA) as described previously [142]. Dim light melatonin onset (DLMO) was calculated as the linearly 
interpolated time of the first sample with a level above $4 \mathrm{pg} / \mathrm{mL}$ that was preceded by a lower value $[36 ; 142]$.

Sleep: Sleep was evaluated actigraphically (Actiwatch, Cambridge Neurotechnology Ltd., Cambridge, UK), which is a valid and reliable method to evaluate sleep and circadian rhythm patterns in children [125]. A sleep log was completed by parents to mark lights out (when the child lies in bed, lights are dimmed and the child is expected to go to sleep) and to provide supplemental data for identifying and rejecting actigraphy inaccuracies, for example due to not wearing the device.

Actigraphs were worn on the non-dominant wrist 24 hours a day for seven consecutive days to estimate sleep onset (start of a period of at least ten minutes of consecutively recorded immobile data following lights out), and total sleep duration (duration from sleep onset until actigraphically estimated wake up time). Data recordings were made at one-minute epochs and scoring was done with a validated automatic scoring algorithm [113] (Actiwatch, Cambridge Neurotechnology Ltd., Cambridge, UK), which compares favorable to other such packages [38], and subsequent manual verification.

\section{Data analysis and statistics}

Differences in 4-repeat allele frequencies between the ADHD-SOI and control group were analyzed with Fisher's exact test and odds ratio (OR) with 95\% confidence intervals (CI). Mean sleep onset, total sleep duration, and DLMO were calculated for each group and their relationship with 4-repeat allele frequency was tested with logistic regression analysis, with age as covariate in the regression model. Furthermore, the relationship of DLMO with age was calculated. Significance level was set at $p \leq 0.05$.

\section{Results}

Subjects

A number of 176 children were referred of which 37 were not diagnosed with ADHD, 33 had no sleep problems, five children were diagnosed with pervasive developmental disorder, four children had an $\mathrm{IQ}<80$, one used methylphenidate, and one used melatonin within one month before participation (Figure 1). Eight children withdrew for a variety of reasons, e.g. including moving or necessity for an urgent start of therapy. Of the remaining 87 ADHD-SOI children, 62 were selected with an onset of insomnia before age three years.

Subsequently, ten children with the latest DLMO values were selected (mean $( \pm$ SD) age $10.1 \pm 1.5$ years). In this group (ADHD-SOI), mean DLMO ( \pm SD) was 
$21: 38 \mathrm{~h} \pm 0: 50 \mathrm{~h}$ whereas in the remaining group of 52 children this was $20: 24 \pm$ 0:51 h. In the ADHD-SOI group, mean ( $\pm \mathrm{SD}$ ) sleep onset was $22: 17 \pm 0: 46 \mathrm{~h}$ and total sleep duration was 9:26 $\pm 0: 40 \mathrm{~h}$.

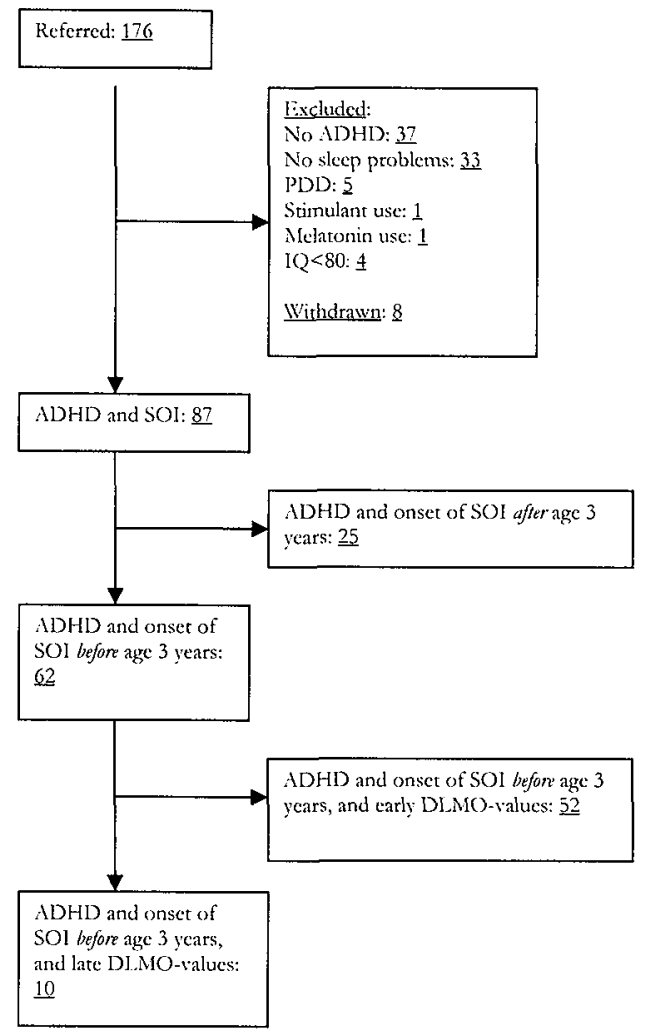

Figure 1. Trial Profile. ADHD: attention-deficit/hyperactivity disorder; SOI: idiopathic chronic sleep onset insomnia; PDD: pervasive developmental disorder; DLMO: dim light melatonin onset. 
Frequency of PER3 VNTR polymorphism and association with sleep onset, total sleep duration and DLMO

The 4-repeat allele frequency was 0.65 in ADHD-SOI and 0.75 in the normal control group ( $p=0.73$ ) (Figure 2), with an odds ratio of 0.62 (CI 0.16-2.4). Frequency of the 4-repeat allele was not significantly related to DLMO ( $\mathrm{R}=0.18$; $p=0.46)$, sleep onset $(\mathrm{R}=0.13 ; p=0.60)$, or sleep duration $(\mathrm{R}=0.14 ; p=0.55)$, and remained non-significant after correction for age. Furthermore, there was no relationship between DLMO and age $(\mathrm{R}=0.12 ; p=0.75)$.

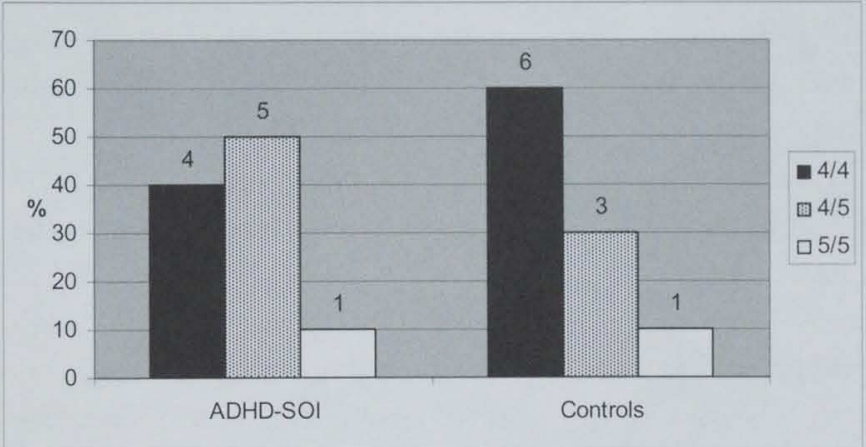

\begin{tabular}{|l|l|l|}
\hline & $\begin{array}{l}\text { ADHD-SOI } \\
(\mathbf{N}=10)\end{array}$ & $\begin{array}{l}\text { Controls } \\
(\mathbf{N}=10)\end{array}$ \\
\hline 4 repeat, No. $(\%)$ & $13(65)$ & $15(75)$ \\
\hline 5 repeat, No. $(\%)$ & $7(35)$ & $5(25)$ \\
\hline
\end{tabular}

Figure 2. The 4- and 5 repeat allele frequencies of the PER 3 clock gene in children with ADHD and idiopathic chronic sleep onset insomnia (ADHD-SOI) and controls.

\section{Discussion}

The present results do not support our initial hypothesis that ADHD-related chronic idiopathic sleep onset insomnia is associated with the 4-repeat allele of the PER3 clock gene. Namely, the frequency of the 4-repeat allele in ADHD-SOI of 0.65 was comparable to the 0.68 found previously in the general population (of the 
northern hemisphere), and not to the 0.88 found in the group of patients with DSPS [16].

We thoroughly selected a group of children with ADHD with characteristics similar to delayed sleep phase syndrome through a measurement of DLMO, which is the most reliable phase marker of the endogenous pacemaker [108]. DLMO was markedly later in the selected ADHD-SOI group than in the remaining children with $\mathrm{ADHD}$ and chronic sleep onset insomnia, as well as compared to previous data in normal children $(19: 45 \pm 0: 60 \mathrm{~h})$ [191] and clinical controls without sleep problems $(20: 07 \pm 0: 64 \mathrm{~h})$ [212]. This is not likely due to age differences since we found no relationship between DLMO and age in children with chronic sleep onset insomnia. Sleep wake rhythms were evaluated rigorously with actigraphy, which is currently the most reliable and valid method to evaluate sleep-wake thythms during longer periods [125]. Since the use of psychotropic medicine was not allowed, a possible influence of these drugs on sleep and DLMO [7;22;193;195;202] can be excluded. The diagnosis of ADHD was made according to well established criteria [13] and clinical practice guidelines [67;91] in order to reduce heterogeneousity of the sample as much as possible.

Nevertheless, there are limitations to this study. Firstly, because of the small sample sizes there is a low power, which implies a relatively high chance of type II errors. i.e. false negative results. Secondly, the control group was not adequately screened for behavioral and sleep problems, which increased the risk that ADHDrelated chronic SOI occurred in this group. Nevertheless, this chance is very low since the current prevalence of ADHD in the Dutch population is 3-5\% [13], while the occurrence rate of chronic sleep onset insomnia within this population was about $11-28 \%$ [50;193].

Since the underlying genetic factors of DSPS may vary [175], further research is needed on other possible genes and polymorphisms that may associate with thythm delays, such as the T1940G polymorphism in exon 15 of the PER3 gene [68]. Furthermore, while multiple genetic factors may contribute to the DSPS phenotype and its manifestation in ADHD-SOI, it remains unknown whether environmental or behavioral factors are involved in DSPS. 
Melatonin treatment for chronic sleep onset insomnia in childhood 

Prediction of melatonin efficacy by pretreatment dim light melatonin onset in children with idiopathic chronic sleep onset insomnia

Van der Heijden, K.B., Smits, M.G., Van Someren, E.J.W., Gunning, W.B. Prediction of melatonin efficacy by pretreatment dim light melatonin onset in children with idiopathic chronic sleep onset insomnia. J Sleep Res., 2005, 14: 187-94. 


\begin{abstract}
Research has shown efficacy of melatonin treatment to advance sleep-wake thythms in insomnia. In healthy adults, direction and magnitude of the phase shift depends on the timing of administration relative to the phase position of the circadian system. Therefore, in the present study we investigated whether in children with chronic sleep onset insomnia (SOI) efficacy of melatonin treatment in the early evening could be predicted from dim light melatonin onset (DLMO), a phase marker of the circadian system. We combined data of two previously published double blind, randomized, placebo-controlled trials in 110 participants, aged 6-12 years. Sleep was actigraphically estimated, and saliva collected, at baseline and in the third week of a four-week treatment period with $5 \mathrm{mg}$ melatonin or placebo at 18:00 or 19:00 h. Primary outcome measures were pre- to post treatment changes in dim light melatonin onset $(\triangle \mathrm{DLMO})$, sleep onset $(\Delta \mathrm{SO})$, sleep latency $(\Delta \mathrm{SL})$, and total sleep duration $(\Delta \mathrm{TSD})$.

Melatonin advanced DLMO with $+1: 12 \mathrm{~h}(p<0.001)$, SO with $+0: 42 \mathrm{~h}$ $(p=0.004)$, SL decreased with 25 minutes $(p=0.019)$, and TSD did not change significantly, as compared to placebo. In the melatonin-treated group, but not in the placebo-treated group, pre-treatment DLMO was significantly related to $\Delta \mathrm{DLMO}(\mathrm{F}(1,29)=7.28, p=0.012)$ and $\Delta \mathrm{SO},(\mathrm{F}(1$, $25)=7.72, p=0.010)$. The time interval between treatment administration and pre-treatment DLMO (INT) was only significantly related to $\triangle \mathrm{SO}$ $(F(1,26)=5.40, p=0.028)$. The results suggest that in children with SOI, the efficacy of early evening melatonin to advance sleep onset and endogenous melatonin onset increases the later the pre-treatment DLMO is.
\end{abstract}




\section{Introduction}

Melatonin is a hormone produced by the pineal gland during the dark phase of the light-dark cycle. When administered exogenously melatonin has proved to be efficacious against various types of insomnia, such as in jet lag [92], night-work [18;185], blindness [179], delayed sleep phase syndrome [105;143], non 24-h sleepwake rhythm [89;132], medical illness [15], and aging [238]. Furthermore, it was effective in insomniac children with mental retardation and neurological disorders [99], and Rett's syndrome [131].

Recently, two placebo-controlled trials we have carried out demonstrated that melatonin therapy was effective in elementary school children with idiopathic chronic sleep onset insomnia (SOI) [190;191].

A reasonable hypothesis of the underlying mechanisms of melatonin is based on the chronobiotic effect, i.e. that exogenous melatonin is a 'Zeitgeber' that phase advances or delays its own secretion depending on the timing of administration [121]. Most likely, this action is mediated by binding to melatonin receptors on neurons of the suprachiasmatic nucleus of the hypothalamus $(\mathrm{SCN})$, the brain's biological clock [226]. The SCN generates not only the circadian rhythm of melatonin, but also other physiological thythms such as of cortisol, leptin, temperature, and the sleep-wake cycle. Given that exogenous melatonin alters the phase of this central clock, one would expect it to shift not only melatonin but also other thythms, as has indeed been found [35;75;138;165;233]. In addition to the chronobiotic effect, mechanisms for direct sleep promoting properties of melatonin have been proposed as well $[35 ; 180 ; 216]$.

In healthy adults, exogenous melatonin shifts circadian thythms according to a phase-response curve (PRC) with a phase-advance zone extending from six hours after, to six hours before the beginning of the subjective day. The magnitude of the phase advance depends on the timing of administration within the phase-advance zone $[120 ; 121 ; 235]$. So far, no studies exist which investigated whether in insomniacs melatonin efficacy can be predicted from the time at which administration occurs within the circadian rhythm. Therefore, in the present study we investigated this in children with SOI using combined data from two previously published placebo-controlled trials in which efficacy was measured actigraphically [190;191]. Actigraphy is a reliable and useful means of studying sleep-wake patterns in children $[14 ; 125]$. In the present study, the circadian phase position was estimated from the dim light melatonin onset (DLMO), which has been proved to be a reliable circadian phase marker [108;119]. 


\section{Methods}

\section{Participants}

Individual patient data of two previously published randomized, placebocontrolled, double blind, clinical trials on the efficacy of melatonin in children with chronic sleep onset insomnia, using similar methodology [190;191], were combined (Fig. 1). Participants were elementary school children with chronic problems falling asleep who were referred to our sleep center by pediatricians and child psychologists in the Netherlands.

Of a total of 110 children, one boy in the placebo group was withdrawn because of an allergic reaction to lactose in the medication. In the melatonin group, parents of nine children withdrew their informed consent during the baseline week. The melatonin treatment group consisted of 31 boys and 15 gitls, with a mean age of $9.8 \pm 1.8$ years and the placebo group of 45 boys and 9 girls with a mean age of 9.7 \pm 1.6 years. All subjects suffered from idiopathic chronic sleep onset insomnia (SOI), which is defined as a sleep onset later than $20: 30 \mathrm{~h}$ for children at age six years, and for older children 15 minutes later per year. Sleep latency needed to be more than 30 minutes and the sleep problems needed to occur more than four days per week on average.

Exclusion criteria were sleep maintenance insomnia (one awakening with a duration of more than 30 minutes or two or more awakenings of more than five minutes summing up to at least 40 minutes, occurring on one or more nights a week, for a period of at least four weeks preceding the start of the trial); disturbed sleep architecture measured by ambulatory polysomnography; mental handicap; severe learning disabilities; any prior use of melatonin; liver diseases; renal failure; use of hypnotics, antidepressants, and neuroleptics; chronic pain; and severe neurological or psychiatric disorders. At enrolment, children were examined by a neurologist (MGS) who specializes in childhood sleep disorders, minimizing the possibility that chronic sleep onset insomnia was due to habits and associations, poor limit settings, fears, improper schedules, medical triggers, and neurological dysfunction.

A group of eight girls and ten boys, aged $8.21 \pm 2.1$ years $(\mathrm{SD})$, without sleep problems, somatic complaints, psychiatric disorders, and psychottopic medication were recruited to serve as a comparison group for normal DLMO values. These were family members of the clinical staff $(n=10)$, or children referred to a mental health clinic for externalizing behavioral problems $(n=8)$, but who did not suffer from sleep problems and did not meet criteria of a DSM-IV disorder [13].

The trials were conducted according to the European Guidelines for Good Clinical Research Practice in children and followed the 1983 revised provisions of 
the 1975 Declaration of Helsinki. The local Medical Ethical Committee of Hospital Gelderse Vallei approved the protocols. The participants' parents gave written informed consent. The sponsors had no role in the study design, data collection, data analysis, data interpretation, or writing of the report.

\section{Procedures}

After a pre-treatment measurement week, trial participants received $5 \mathrm{mg}$ melatonin or identically looking placebo at 18:00 h [190], or 19:00 h [191]. Patients and staff were blind to the allocated treatment.

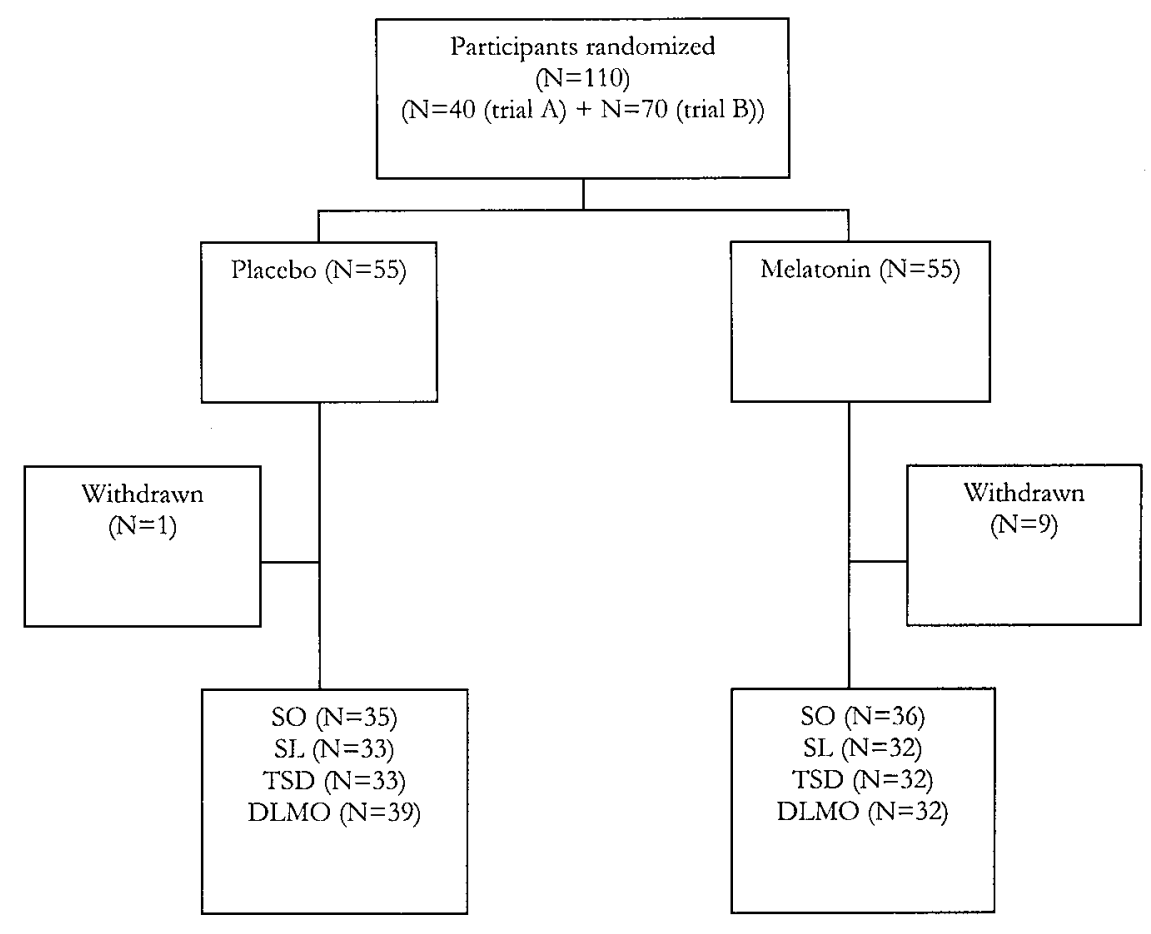

Figure 1. Trial profile (Trial A [190], trial B [191]). SO: sleep onset (actigraphy), SL: sleep latency (actigraphy), TSD: total sleep duration (actigraphy), DLMO: dim light melatonin onset (salivary). 


\section{Assessment of sleep}

In the pre-treatment week and in the fourth treatment week subjects wore an actigraph (Gähwiler Electronics, Hombrechtikon, Switzerland $®$ [190]), (Cambridge Neurotechnology $\left.{ }^{\circledR}[191]\right)$ on their non-dominant wrist for two consecutive nights. Sleep parameters were estimated from actigraphy data by automatic scoring (Gähwiler Electronics, Hombrechtikon, Switzerland (B) [190]), (Cambridge Neurotechnology ${ }^{\circledR}$ [191]), and subsequent manual verification. Two groups of parameters were distinguished: 1) reference times: sleep onset ( $\mathrm{SO}$ ) defined as the clock time one falls asleep, and DLMO, 2) interval parameters: sleep latency (SL) defined as the time it takes to fall asleep after lights out, total sleep duration (TSD) defined as the duration from sleep onset until awake time, and INT: the time interval between administration and pre-treatment DLMO (pre-treatment DLMO minus clock time of administration). During the trials, the children were allowed to go to bed when they felt tired rather than at a set time.

\section{Assessment of dim light melatonin onset}

Salivary samples were collected hourly from 19:00 to 23:00 h by chewing on a cotton plug (Salivetten, Sarstedt Nümbrecht, Germany) for 1 minute. These saliva collection time points were chosen because previous studies had shown that melatonin onset could be expected to occur in that time interval [143]. In both trials, saliva was collected on the last day of the pre-treatment week and fourth treatment week. Salivary melatonin concentrations were assayed with radioimmunoassay (RIA) as described previously [142]. During the measurement period the children remained in bed while the curtains were closed to prevent suppression of melatonin secretion by bright light [28], however, dim light was allowed. Dim light melatonin onset (DLMO), defined as the first time at which salivary melatonin reached $4 \mathrm{pg} / \mathrm{mL}$, was calculated as the linearly interpolated time of the first sample above $4 \mathrm{pg} / \mathrm{mL}$ that was preceded by a lower value [36;142]. The trial participants did not take the study medication during the night of saliva collection.

\section{Statistical analysis}

Normal comparison group: Mean DLMO was calculated for the entire group, and for age groups 6-9 and 10-13 years. Differences in DLMO between age groups were analyzed with Mann-Whitney Test. Relationship between DLMO and age was analyzed with linear regression analysis. 
Trial participants: General linear model (GLM) repeated measures analysis of variance was used to test between-group differences in pre- to post treatment changes of SO, SL, TSD, and DLMO ( $\triangle S O, \triangle S L, \triangle T S D$, and $\triangle D L M O)$. The circadian phase position at the time of administration was determined by calculating INT. GLM univariate analysis with $\triangle \mathrm{SO}, \triangle \mathrm{SL}, \triangle \mathrm{TSD}$, and $\triangle \mathrm{DLMO}$ as dependent variables and age, pre-treatment DLMO, and INT respectively as covariates, was performed separately for the placebo and melatonin group. Pearson's correlation coefficients between $\triangle \mathrm{SO}$ and $\triangle \mathrm{DLMO}$ were computed.

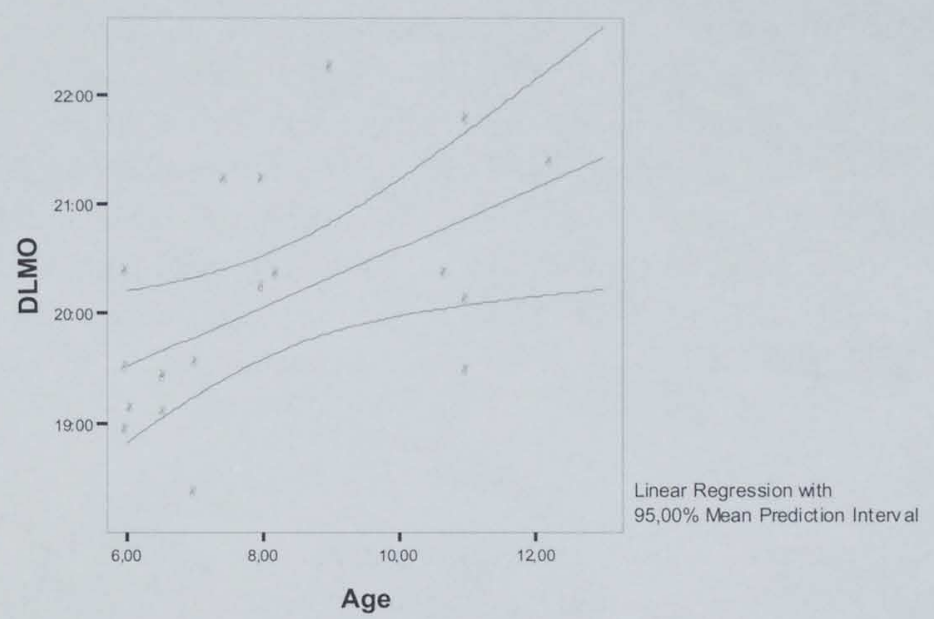

Figure 2. Relationship of dim light melatonin onset with age in 18 normal children.

In order to evaluate a possible effect of systematic differences caused by the use of two types of actigraphs and their sleep-scoring software, we introduced this as a dummy coded (0-1) covariate. Furthermore, we evaluated differences between the two trials in sex distribution, mean age, pre-treatment dim light melatonin onset (DLMO), and pre-treatment sleep characteristics (sleep onset, sleep latency, and total sleep duration) to evaluate whether the datasets were homogeneous. Statistical significance was set at $p \leq 0.05$. Statistical analyses were conducted using SPSS (V.11.5.1, SPSS Inc, Chicago, Ill). 


\section{Results}

Normal comparison group

Mean ( \pm SD) DLMO was 20:07 $\pm 1: 04 \mathrm{~h}$ in the control group of healthy and behaviorally disturbed but well sleeping children. The linear regression line in Fig. 2 indicates that DLMO delays with increasing age in this group: a $0: 16 \mathrm{~h}$ delay for every additional year of age $\left(\mathrm{R}^{2}=0.28 ; p=0.024\right)$.

\section{Trial participants}

Due to technical problems, the actigraphy data of 19 children in the placebotreated group and 10 in the melatonin-treated group could not be analyzed. In two placebo-treated children and four melatonin-treated children, SL or TSD could not be analyzed because of missing lights-out time or wake-up time. Saliva was not produced or medication was taken on the night when saliva was collected in 15 children of the placebo-treated group and 14 of the melatonin-treated group. Mean ( $\pm \mathrm{SD})$ DLMO in the trial participants was 20:55 $\pm 1: 00 \mathrm{~h}$. In contrast to the normal comparison group, in children with chronic SOI (pre-treatment) DLMO was not significantly related to age. There were no significant differences in pretreatment SO, SL, TSD, and DLMO between subjects assigned to the melatonin versus placebo treatment conditions.

\section{Treatment effects}

As can be seen in Table 1, pre- to post treatment changes in SO, SL, and DLMO $(\triangle \mathrm{SO}, \Delta \mathrm{SL}$, and $\triangle \mathrm{DLMO})$ after melatonin treatment were significantly different from those after placebo ( $p=0.004 ; p=0.019 ; p<0.001$, respectively). There was no significant correlation between $\triangle \mathrm{SO}$ and $\triangle \mathrm{DLMO}$.

In the melatonin treated group, we found a significant relationship between pretreatment DLMO and $\triangle \mathrm{SO}$ and $\triangle \mathrm{DLMO}(\mathrm{F}(1,27)=7.72 ; p=0.010$, and $\mathrm{F}(1,31)=$ $7.27 ; p=0.012$, respectively), but not with $\Delta$ SL and $\Delta \mathrm{TSD}$ (Table 2). However, in the placebo group pre-treatment DLMO was not significantly related to any pre- to post treatment change. The shift in sleep onset $(\Delta \mathrm{SO})$ was plotted against pretreatment DLMO in a scatterplot (Fig. 3). The linear regression line of the melatonin treated group entered the phase advance zone (i.e. above the zero line of the $Y$ axis) at $19: 32 \mathrm{~h}$ with a slope of 0.41 , indicating that when pre-treatment DLMO occurred later than 19:32 $\mathrm{h}$, the advance in sleep onset after melatonin treatment increased with 0:25 $\mathrm{h}$ for every one-hour delay of pre-treatment DLMO. As shown in Figure 4, the advance in DLMO increased with $0: 16 \mathrm{~h}$ for every onehour delay of pre-treatment DLMO. 
Table 1. Mean pre- and post treatment values of DLMO, sleep onset, sleep latency, and total sleep duration.

\section{Melatonin Placebo}

\begin{tabular}{|c|c|c|c|c|c|}
\hline $\begin{array}{l}\text { Pre- } \\
\text { treatment }\end{array}$ & $\begin{array}{l}\text { Post- } \\
\text { treatment }\end{array}$ & $\begin{array}{l}\text { Change } \\
\text { (pre - } \\
\text { post) }\end{array}$ & $\begin{array}{l}\text { Pre- } \\
\text { treatment }\end{array}$ & $\begin{array}{l}\text { Post- } \\
\text { treatment }\end{array}$ & $\begin{array}{l}\text { Change } p \text {-Value } \\
\text { (pre- } \\
\text { post) }\end{array}$ \\
\hline
\end{tabular}

DLMO: dim light melatonin onset (clock time); SO: sleep onset (clock time), SL: sleep latency (minutes), TSD: total sleep duration (minutes).

* Significant difference between melatonin and placebo $(\phi \leq 0.05)$, two-sided test.

As for placebo, the regression lines in Fig. 3 and 4 remained close to the zero line of the $\mathrm{Y}$ axis, indicating that the shift by placebo was negligible.

The mean value of INT, the time interval between administration and pretreatment DLMO was 2:33 $\pm 1: 23 \mathrm{~h}(\mathrm{SD})$ in the melatonin group and 2:26 $\pm 0: 58 \mathrm{~h}$ (SD) in the placebo group. In the melatonin group, INT was significantly related only to $\Delta \mathrm{SO}(\mathrm{F}(1,26)=5.40 ; p=0.028)$ and not to $\triangle \mathrm{DLMO}$ (Table 2). In the placebo group, INT was not related to pre- to post treatment changes in any parameter.

Fig. 5 shows the relationship between treatment-induced phase shifts in sleep onset and pre-treatment DLMO. For an intake of melatonin at longer than 0:46 hours before DLMO, the linear regression line of the melatonin treated group predicted that - within the obtained range of intervals - the advance of sleep onset increased by $0: 19 \mathrm{~h}$, for every one-hour increase in the interval between melatonin administration and pre-treatment DLMO. However, the results predict a delay of sleep onset when this interval was shorter than $0: 46 \mathrm{~h}$. 
Table 2. Effects of covariates age, pre-treatment DLMO, and INT on pre- to post treatment changes in DLMO, sleep onset, sleep latency, and total sleep duration.

\begin{tabular}{llll}
\hline & Covariate & Melatonin & Placebo \\
\hline$\Delta$ DLMO & Age & $\mathrm{F}(1,31)=3.77, p=0.062$ & $\mathrm{~F}(1,38)=0.008, p=0.928$ \\
& Pre-treatment DLMO & $\mathrm{F}(1,31)=7.27, p=0.012 *$ & $\mathrm{~F}(1,38)=0.874, p=0.356$ \\
& INT & $\mathrm{F}(1,30)=1.309, p=0.262$ & $\mathrm{~F}(1,36)=0.741, p=0.395$ \\
$\Delta \mathrm{SO}$ & Age & $\mathrm{F}(1,27)=0.921, p=0.346$ & $\mathrm{~F}(1,27)=0.466, p=0.501$ \\
& Pre-treatment DLMO & $\mathrm{F}(1,27)=7.72, p=0.010 *$ & $\mathrm{~F}(1,27)=0.370, p=0.549$ \\
& INT & $\mathrm{F}(1,26)=5.40, p=0.028 *$ & $\mathrm{~F}(1,25)=0.001, p=0.975$ \\
$\Delta \mathrm{SL}$ & Age & $\mathrm{F}(1,23)=0.035, p=0.854$ & $\mathrm{~F}(1,26)=0.362, p=0.553$ \\
& Pre-treatment DLMO & $\mathrm{F}(1,23)=0.121, p=0.731$ & $\mathrm{~F}(1,26)=0.460, p=0.504$ \\
& INT & $\mathrm{F}(1,23)=0.249, p=0.623$ & $\mathrm{~F}(1,24)=0.021, p=0.887$ \\
$\Delta \mathrm{TSD}$ & Age & $\mathrm{F}(1,23)=2.12, p=0.161$ & $\mathrm{~F}(1,26)=0.883, p=0.357$ \\
& Pre-treatment DLMO & $\mathrm{F}(1,23)=0.353, p=0.559$ & $\mathrm{~F}(1,26)=1.05, p=0.315$ \\
& INT & $\mathrm{F}(1,23)=0.014, p=0.908$ & $\mathrm{~F}(1,24)=0.398, p=0.534$ \\
\hline
\end{tabular}

${ }^{*} p \leq 0.05$, two-sided; $\triangle \mathrm{DLMO}$ : change in dim light melatonin onset, $\triangle \mathrm{SO}$ : change in sleep onset, $\triangle S L$ : change in sleep latency, $\triangle T S D$ : change in total sleep duration, INT: interval between the time of medication administration and pre-treatment DLMO.

The regression line of the melatonin treated group in Fig. 6 predicted a DLMO phase advance of approximately one hour, irrespective of the magnitude of INT.

The results showed that age was not a predictor of treatment-induced changes in SO, SL, 'TSD, and DLMO. Analysis of pre-treatment sleep measures revealed no significant effect of actigraph type (Gähwiler or Actiwatch) on the means of the three sleep parameters $\mathrm{SO}(21: 53$ vs. $21: 57 \mathrm{~h} ; \mathrm{F}(1,70)=0.81, p=0.78)$, SL (61 vs. 58 $\min ; \mathrm{F}(1,65)=0.06, p=0.81$ ), and TSD (572 vs. $577 \mathrm{~min} ; \mathrm{F}(1,65)=0.11, p=0.75$ ) allowing the pooling of the two datasets.

Furthetmore, there were no statistical differences between the two trials in sex distribution ( $71 \%$ vs. $79 \%$ boys; $p=0.20)$, mean age $(9,8$ vs. 9,7 yrs. $p=0.78)$, pretreatment dim light melatonin onset (DLMO) (21:07 vs. 20:48 h; $p=0.18)$, and pretreatment sleep characteristics (SO: $21: 53$ vs. $21: 57 \mathrm{~h} ; p=0.78$; SL: 61 vs. 58 mins; $p=0.81$; and TSD: 572 vs. 577 mins; $p=0.75$ ), indicating that the two datasets were satisfactorily homogeneous. 

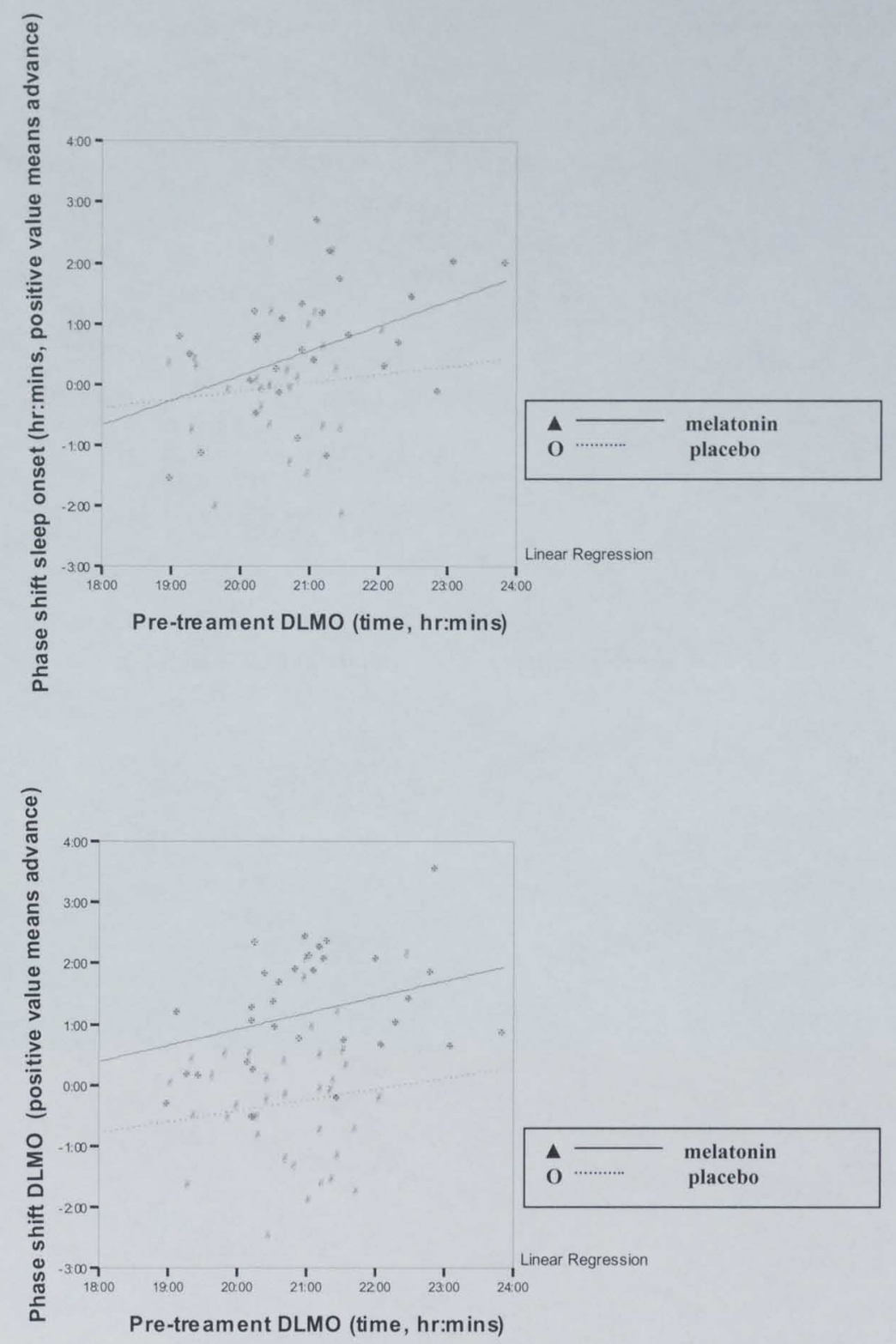

Figure $3 \& 4$. Relationship of pre-treatment dim light melatonin onset (DLMO) with phase shifts after treatment of sleep onset and DLMO respectively. 

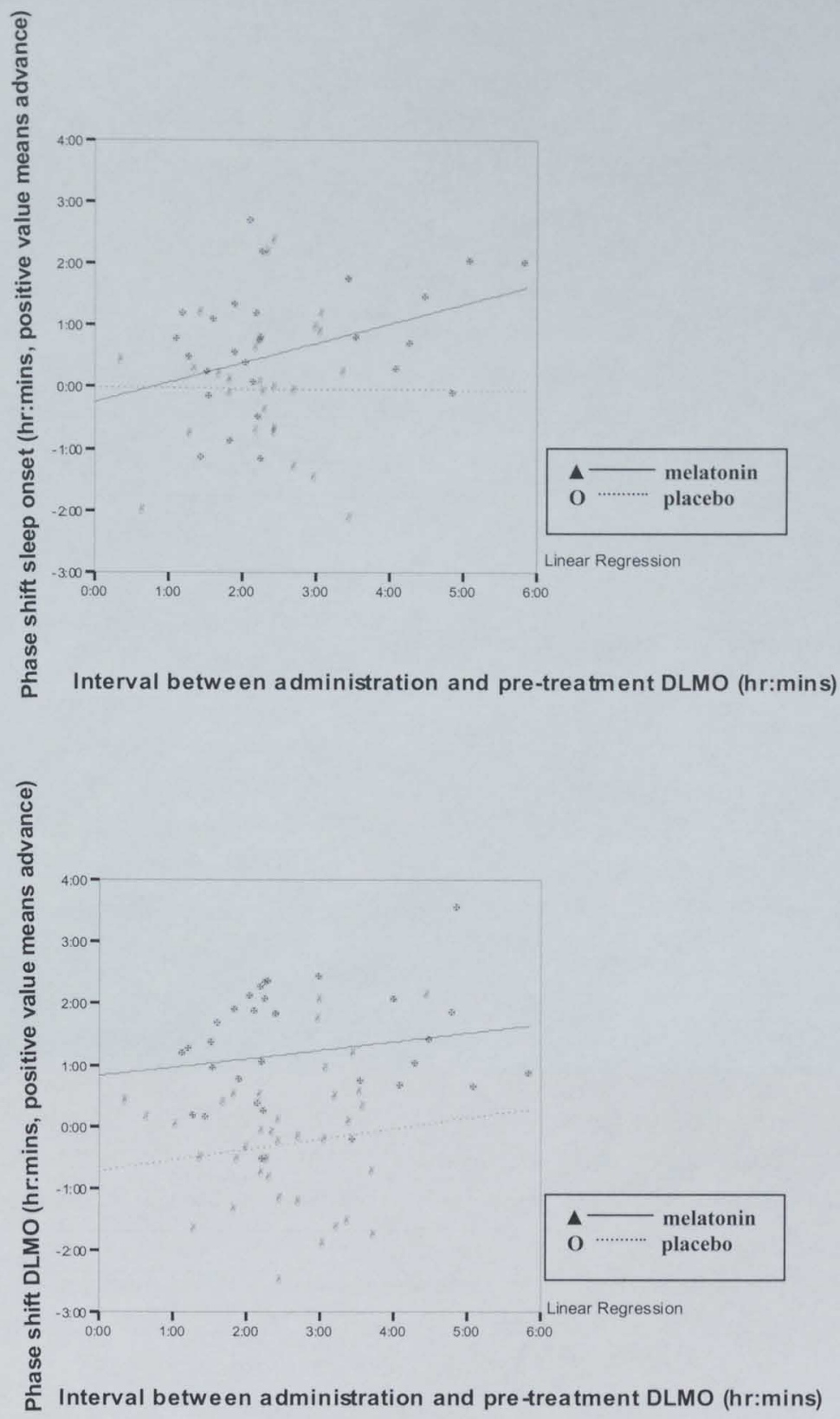

Figure $5 \& 6$. Relationship of the time interval between administration and pre-treatment dim light melatonin onset (DLMO) with phase shifts after treatment of sleep onset and DLMO respectively. 


\section{Discussion}

Our study is the first to show that in children with chronic sleep onset insomnia the efficacy of early evening melatonin treatment to advance sleep onset can be predicted by circadian phase marker DLMO. Since the relationship between pretreatment DLMO and efficacy could not be found in the group of patients who were assigned to placebo treatment, a confounding involvement of regression to the mean is unlikely. The pre- to post treatment changes in sleep latency and total sleep duration were not related to pre-treatment DLMO. This might be explained by the marginal effect that melatonin had on sleep duration, which was also found in several other studies in insomniacs [83;105;190;191].

The widely held view is that the phase shifting effect of melatonin depends on the circadian phase position at the time of administration, which is in our study indicated by the interval between administration and pre-treatment DLMO. The present results show that the earlier melatonin is administered within a window of 0:46 to 6:00 hours of DLMO, the larger the phase-advance of sleep onset. This is in accordance with the phase-response curve in healthy adults which showed a maximal phase advance - however of DLMO - when administration occurred approximately five hours prior to DLMO, and showed decreasing phase advances until (phase-delay) cross-over points were approached at eight hours before and four hours after DLMO [120;121;235]. According to the present results, the children in our study who did not respond to melatonin treatment and who showed an early pre-treatment DLMO might have required an earlier administration of melatonin. Unfortunately, melatonin administration in the afternoon is undesired because of potential soporific effects.

We found that pre-treatment DLMO was a better predictor of efficacy than the interval between administration and pre-treatment DLMO. Two factors may be involved in this somewhat surprising finding. Firstly, the time of administration was nearly fixed so that the magnitude of the interval was mainly determined by pretreatment DLMO. Secondly, the calculation of parameter INT occurred by subtracting the time of administration from pre-treatment DLMO which introduced an additional error in the time of melatonin intake to the error in measurement of DLMO. Consequently, the variable INT approaches the variable pre-treatment DLMO but suffers from additional measurement error.

Three of our findings support the premise that melatonin acts as a 'Zeitgeber': 1) the phase-advancing effect of melatonin on both sleep onset and melatonin onset, 2) the positive relationship between pre-treatment melatonin onset and change in sleep onset and melatonin onset, 3) the presence of a phase-response relationship for the advance in sleep onset such as predicted by the previously reported phase- 
response curve for adults. In contrast, we did not find a positive relationship between the change in DLMO and the change in sleep onset, which is not in line with what one should expect if 'Zeitgeber' were the only mechanism. Hence, additional mechanisms might have been involved such as a soporific effect of distal vasodilation i.e. increase in skin blood flow and consequently skin temperature [215;216], the reduction of core body temperature [61;96], and a possible influence at the GABA chloride channel complex [180].

In photoperiodic nonhuman mammals, the secretion of melatonin from the pineal gland plays a major role in regulating reproductive physiology [77;234]. In humans, relationships between melatonin and the status of the reproductive system have been noted [188], however, whether they are functionally related remains to be determined [128;168]. So far, no influence of exogenous melatonin on puberty has been reported, but neither has there been any considerable effort to actively investigate this issue. In the children who participated in our earlier reported placebo-controlled trials [190;191] (most used melatonin during several years after the trial), adverse effects have not been reported until now. In an ongoing study we are systematically collecting data on the long-term use of melatonin in both children and adults. As long as melatonin's long-term effects in pre-pubertal children have not been studied systematically, its use in children should only be applied when correction of underlying causes was not achievable or effective. Our results can aid the ethical decision whether or not to treat children with chronic sleep onset insomnia with melatonin, since its likely efficacy can be predicted by the circadian phase marker DLMO.

Bright light therapy may be an alternative to melatonin treatment in juvenile idiopathic chronic sleep onset insomnia. Many children, however, already expose themselves to morning light because they walk or bike to school, usually around 8:00 h. At that time, the light intensity outdoors is in the Netherlands usually much higher than the 3000 lux used with light therapy. We are presently investigating the possibility to replace melatonin after a few weeks of treatment by early morning light therapy.

To conclude, our results suggest that in elementary school children with idiopathic chronic sleep onset insomnia, the effectiveness of early evening melatonin treatment to advance sleep onset and the endogenous onset increases the more delayed the endogenous onset is before commencement of treatment. 
Childhood chronic sleep onset insomnia and late sleep onset: what's the difference?

Van der Heijden, K.B., Smits, M.G., Van Someren, E.J.W., Gunning, W.B. Childhood chronic sleep onset insomnia and late sleep onset: what's the difference? I Sleep Res., 2005, 14: 197-199. Reply on Jenni, 2005, 14: 195-7). 
In his comments on our atticle [212], Dr Jenni claims that our definition of childhood idiopathic chronic sleep onset insomnia (SOI) does not take into account inter- and intracultural variation [103]. He asserts that idiopathic chronic SOI is not a circadian thythm sleep disorder, but "rather an extreme variant of biologically driven normal behavior" that does not fit the adult interest. Therefore, he is concerned about melatonin treatment of childhood idiopathic chronic SOI.

We regret that dr Jenni gives an incomplete description of our criteria for chronic sleep onset insomnia (SOI). He focuses his critics on our lower limit time criteria of sleep onset, while omitting the core criteria. Firstly, he ignores the complaint of not being able to fall asleep. In our studies, children needed to suffer from chronic complaints of insomnia, on at least four weekdays per week, for longer than one year [190;191], which Dr Jenni fails to mention. These criteria are much more strict than all diagnostic classification systems $[13 ; 64 ; 69]$ and quantitative criteria for insomnia in adults [123]. Secondly, he ignores our criterion that the latency between lights-off time and sleep onset (sleep latency) needs to exceed 30 minutes. Thirdly, Dr Jenni forgets that our criteria require that these problems are persistent in the way that they did not improve after improving bedtime routines, sleep schedules, evening events (e.g., naps), and bedroom qualities for longer than three weeks, as supervised by local general practitioners or sleep specialists [190;191].

With respect to the complaints of falling asleep, Dr Jenni claims sleep onset insomnia is a problem of parents without addressing any concerns on the potential problems for the child such as on health, mood, behavior, and cognition [58;205;231]. In fact, most children with chronic SOI aged 6-12 years complain of problems falling asleep themselves and, in addition, often complain of problems waking up and daytime tiredness or attention deficiency. This is supported by our finding that children with SOI showed a significantly impaired general health and daytime functioning as compared to the Dutch child population means (Functional Status II total and illness-specific: $p<0.0001$; RAND General Health Rating Index: $p=0.005$ ) [191]. Moreover, treatment of the childhood insomnia significantly improved general health and daytime functioning [191]. Dr Jenni's claim that chronic SOI is only a problem of parents and not of the child is a general negligence of a pivotal criterion that is included in all current classification systems, namely, that daytime dysfunction is required in order to make a diagnosis of insomnia $[13 ; 64 ; 69]$.

Dr Jenni compares our lower limit time criteria for SOI with average bedtimes in other cultures, which is a serious misunderstanding. Our criteria [190;191;212] do not represent average bedtimes but set the minimal requirements as to sleep onset. 
To show that there are large differences between such minimal requirements and actual sleep onset times we have depicted the average sleep onset times at age 6 to 12 years in Dr Jenni's plot on reported bedtime in Swiss children (figure 1). As can be seen, the average sleep onset in children with chronic sleep onset insomnia is above the $90^{\text {th }}$ or $98^{\text {th }}$ percentile of the reported bedtimes in the Swiss child population. Furthermore, sleep onset of seven-year old insomniacs in our study $(21: 49 \pm 0: 56 \mathrm{~h})$ markedly exceed all other sleep onset times in different cultures that Dr Jenni shows in his comments (max. 21:15 h). Our other comments on Dr Jenni's table of sleep onset times (Table 1; Jenni, 2005 [103]) are: 1) he ascribes the differences in sleep onset times to differences in culture but does not consider possible influences of differences in design and methodology. Furthermore, all studies were performed by different groups, with different instruments and without objective measures of sleep such as actigraphy or polysomnography; 2) the differences in sleep onset between the studies may be due to differences in how parents estimate their child's sleep.

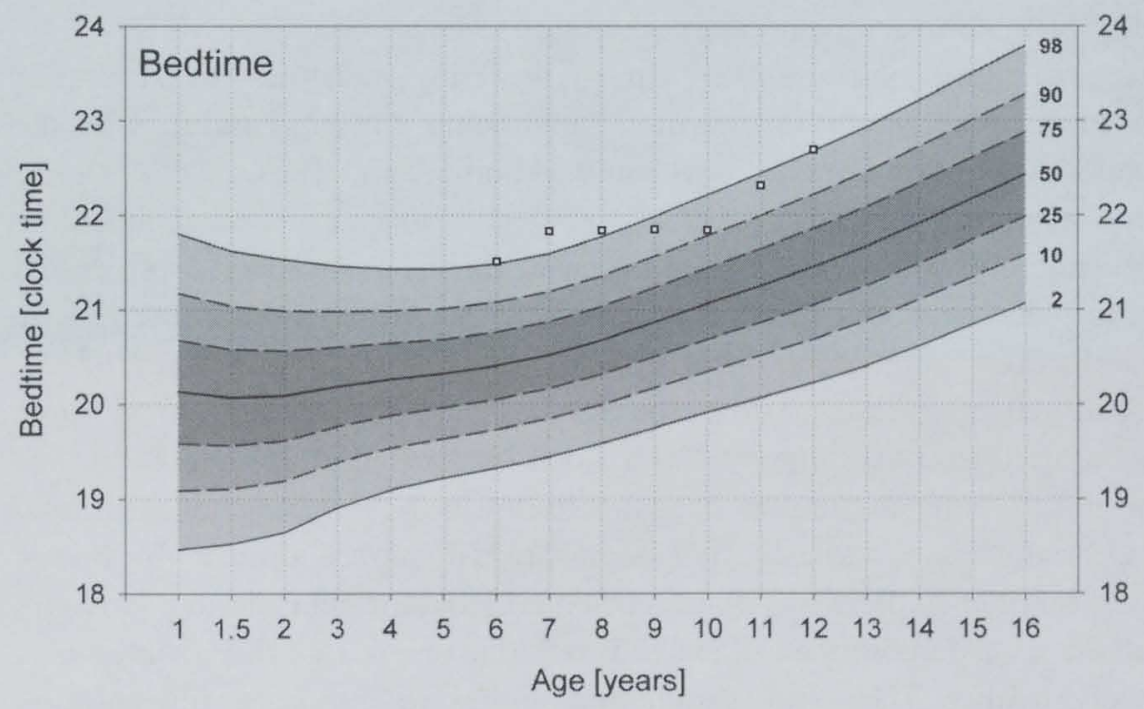

Figure 1. Average actigraphic sleep onset in Dutch children with idiopathic chronic sleep onset insomnia $(n=110)$ aged 6-12 years (square dots) plotted in Gaussian percentiles for reported bedtime in Swiss children (from [103]). 
For instance, parents in the US may know less about their child's real sleep than Chinese parents because of the high frequency of room sharing or bed sharing with parents in Chinese children [126]; 3) sleep latency, total sleep time, wake up time, and naps are not shown which makes comparison with our data impossible. Nevertheless, we agree with Dr. Jenni that, before research diagnostic criteria on chronic childhood sleep onset insomnia (SOI) have been derived, caution should be exercised to adopt our criteria in other cultures.

Currently, melatonin treatment in children is a topic that receives extensive discussion. Therefore, we welcome the opportunity to elaborate on this issue. Dr Jenni makes two remarks, the first pertaining to the higher rate of withdrawal in the melatonin treatment group. As was mentioned in our article [191] these parents withdrew informed consent during the baseline week (before commencement of treatment) because they heard enthusiastic reports on the effectiveness of melatonin. Therefore, their withdrawal of informed consent was not related to the intake of melatonin. Secondly, Dr Jenni questions the purpose of melatonin treatment as it has no effect on sleep time. Melatonin administered in the early evening typically shifts sleep-wake rhythm without significant change of sleep duration. However, some reports showed that melatonin can improve (subjective) sleep quality in insomniacs while sleep duration remains unaffected [76;97;238]. Nevertheless, the most important reason to prescribe melatonin in insomniac children is to improve health and daytime functioning, probably induced by the shift in sleep-wake rhythm or perhaps by other, yet unknown, effects.

The results of our meta-analysis [212] suggest that melatonin treatment should be confined to children with a delayed sleep phase syndrome as indicated by a delayed value of dim light melatonin onset [212]. Delayed sleep phase syndrome can be regarded a disorder when one is not able to adjust a delayed rhythm of the circadian pacemaker to the desired sleep-wake rhythm, resulting in daytime dysfunction. A well-sleeping individual with a delayed biological rhythm working as a night porter, or feeling at comfort in a subculture with late evening and nocturnal activities, is clearly not disordered. We demonstrated that children with chronic SOI show discordance between their biological thythm and desired rhythm, resulting in daytime impairment. Flexibility or adjustment of school times may improve concordance [71], and should be addressed by governmental and educational organizations. We showed that melatonin treatment is an effective and safe alternative in the short term. However, we agree with Dr Jenni that caution should be exercised in long-term prescribing of melatonin before safety of such prescription has been adequately investigated. Melatonin treatment should be administered at the lowest possible dose, should be stopped (at least) once a year to 
evaluate whether it is still necessary, and should be prescribed preferably within clinical trials to fully establish its benefits and risks as soon as possible.

If anything, our study has actually narrowed the application spectrum for the use of melatonin in children, by showing that clinically relevant efficacy can be expected primarily in children with a delayed sleep phase syndrome as indicated by a delayed value of dim light melatonin onset. 
, 


\section{9}

Effect of melatonin on sleep, behavior, cognitive performance, and quality of life in children with
attention/deficit-hyperactivity disorder and chronic sleep onset insomnia

Van der Heijden, K.B., Smits, M.G., Van Someren, E.J.W., Ridderinkhof, K.R., Gunning, W.B. Effect of melatonin on sleep, behavior, cognitive performance, and quality of life in children with attention/deficithyperactivity disorder and chronic sleep onset insomnia. Revised manuscript resubmitted. 


\begin{abstract}
Objective. To investigate the effect of melatonin on sleep, behavior, cognition, and quality of life in children with attention-deficit/hyperactivity disorder (ADHD) and chronic sleep onset insomnia. Method. 105 medication-free children, aged 6-12 years, with diagnosed ADHD and chronic sleep onset insomnia participated in a randomized, double blind, placebo-controlled trial using 3 or $6 \mathrm{mg}$ melatonin (depending on body weight) or placebo for four weeks. Primary outcome parameters were actigraphy-derived sleep onset and total time asleep, and salivary dim light melatonin onset. Results. Melatonin advanced sleep onset as compared to placebo (mean ( $\pm \mathrm{SD}$ ): $-0: 27 \pm 0: 48 \mathrm{~h}$ vs. $+0: 10 \pm 0: 37 \mathrm{~h} ; p<0.0001)$ and advanced dim light melatonin onset as compared to placebo $(-0: 44 \pm 1: 07 \mathrm{~h}$ vs. $+0: 13 \pm 0: 59 \mathrm{~h} ; p<0.0001)$. Total time asleep increased in the melatonin group as compared to placebo (+19.82 $\pm 61.90 \mathrm{~min}$ vs. $-13.58 \pm 50.60 \mathrm{~min}$; $p=0.01$ ). Melatonin showed no significant effect on measures of behavior, cognition, and quality of life and demonstrated no significant adverse events. Conclusion. Melatonin advanced circadian thythms of sleep-wake and endogenous melatonin and enhanced total time asleep in children with ADHD and chronic sleep onset insomnia, however, no effect was found on problem behavior, cognitive performance, or quality of life.
\end{abstract}




\section{Introduction}

Attention-deficit/hyperactivity disorder (ADHD) is characterized by severely disabling levels of inattention, hyperactivity/impulsivity, or both, and is one of the most common psychiatric disorders of childhood [13]. Approximately one third of medication-free ADHD children suffers from chronic sleep onset insomnia [50;193]. In children with $A D H D$, this persistent disability to fall asleep at the desired time in the evening might exacerbate daytime mood-, behavioral-, and/or cognitive problems [181].

The safety and efficacy of melatonin treatment for chronic sleep onset insomnia in non-ADHD children has been documented in several studies [190;191;212]. However, there have been no studies of melatonin efficacy in medication-free ADHD children with chronic sleep onset insomnia, although this patient group is of special interest for two reasons. Firstly, medication-free ADHD children with chronic sleep onset insomnia have a delayed evening rise in endogenous melatonin levels (i.e. dim light melatonin onset) [213] and such phase-delay predicted a stronger sleep phase-normalizing effect of exogenous melatonin in non-ADHD children [212]. Secondly, because treatment of sleep-related disorders other than insomnia improves daytime function in ADHD children $[8 ; 79 ; 104 ; 225]$, the treatment of insomnia may be expected to do so as well and thus may have important consequences for ADHD treatment strategies in health care settings.

We conducted a double-blind, randomized, placebo-controlled, multicenter trial to investigate whether melatonin treatment could improve objective and subjective measures of sleep, behavior, cognitive performance, and quality of life in medication-free children diagnosed with ADHD and chronic sleep onset insomnia.

\section{Method}

\section{Subjects}

176 children with possible ADHD were referred for participation in the present trial by seven Dutch community mental health institutions and three pediatric departments of non-academic hospitals to out-patient clinics for sleep-wake disorders of the Gelderse Vallei general hospital and Kempenhaeghe. A further 28 children were recruited through advertisements in magazines, newspapers, or at the Dutch ADHD patient support center. A total of 107 out of these 204 children was entolled in the present study after diagnostic evaluation (Fig. 1). Baseline data of two-third of these participants were presented elsewhere [213]. Inclusion criteria were: age 6-12 years; diagnosis of ADHD and chronic sleep onset insomnia; and written informed consent, obtained from the participating children's parents. Exclusion criteria were: total IQ less than 80, pervasive developmental disorder, 
chronic pain, known disturbed hepatic or renal function, epilepsy, earlier use of melatonin and use of stimulants, neuroleptics, benzodiazepines, clonidin, antidepressants, hypnotics, or beta blockers within four weeks before enrolment.

\section{Clinical assessment}

Rigorous clinical diagnostic assessments were conducted on all 204 children, based on the Diagnostic and Statistical Manual of Mental Disorders, Fourth Edition (DSM-IV) [13]. Diagnostic assessment was performed by a psychologist (KBH) and board-certified child and adolescent psychiatrist (WBG). ADHD was diagnosed conform the guidelines of the American Academy of Pediatrics [2], and the American Academy of Child and Adolescent Psychiatry [67], and included clinical history; Diagnostic Interview Schedule for Children-Parent form (DISC-P) [146]; Child Behavior Checklist [6]; and Teacher's Report Form [5]. The following ADHD-subtypes were determined: 1) predominantly inattentive type (ADHD-I) assigned to children who displayed more than six inattentive symptoms, but less than six hyperactive/impulsive symptoms; 2) predominantly hyperactive/impulsive type (ADHD-HI) for children with more than six hyperactive/impulsive but less than six inattentive symptoms; and 3) combined type (ADHD-C) for children with more than six symptoms of each set of symptoms. A shortened IQ test (WISC-III Block Design, Vocabulary [228]) was administered when IQ had not been assessed formerly and school performance had been subaverage in the last three years.

Chronic sleep onset insomnia was defined as: 1) complaints of sleep onset problems by parents and/or child; 2) occurrence on at least four weekdays per week, for longer than one year; 3) average sleep onset later than 20:30 $\mathrm{h}$ for children at age six years, and for older children 15 minutes later per year; 4) average sleep latency exceeding 30 minutes [190;191;212;214]. The diagnostic procedures included clinical history; one-week 24-hour actigraphy measurement; Dutch Sleep Disorders Questionnaire [199]; and Children's Sleep Hygiene Scale [84].

\section{Study design and conduct}

A 4-week tandomized, double-blind, placebo-controlled study, immediately following a one-week baseline period, was conducted between November 2001 and June 2005. The protocol was approved by the institutional review board at each center, as a multicenter trial by the Central Committee on Research Involving Human Subjects, and was registered in the International Standard Randomized Controlled Trial Number Register (ISRCTN-47283236). The trial was performed according to the European Guidelines for Good Clinical Research Practice in children (Committee for Proprietary Medicinal Products, 1997), and followed the 
1983 revised provisions of the 1975 Declaration of Helsinki. Sponsors had no role in the study design, data collection, data analysis, data interpretation, or writing of the teport.

Children whose parents gave informed consent were randomly assigned (in a 1:1 ratio) to receive melatonin ( $3 \mathrm{mg}$ when body weight $<40 \mathrm{~kg}$ [ $\mathrm{n}=44] ; 6 \mathrm{mg}$ when body weight $>40 \mathrm{~kg}[\mathrm{n}=10]$ ), in fast-release tablets (Pharma Nord, Denmark), or identical-appearing placebo tablets, at 19:00 h. We used a pharmacological dose that had previously been proved effective and safe in children [190;191] and employed a fixed time $(19: 00 \mathrm{~h})$ of administration for all participants. Assessment of treatment compliance was performed at a visit after three weeks of treatment. All measurements took place at baseline and during the fourth treatment week. Parents were called weekly to monitor progress, to discuss any possible problems, and were instructed to call the principal researcher at any time for help or information, or to report possible adverse events. During the entire trial period, the children were allowed to go to bed whenever they felt tired rather than being tied to a scheduled bedtime. Participants were not allowed to start or change therapeutic interventions during the entire trial period that could influence measures obtained in the present study. No assessments took place during or within three days following holidays or clock time transitions.

\section{Outcome measures}

Sleep. Sleep was estimated using actigraphy (Actiwatch, Cambridge Neurotechnology Ltd, Cambridge, UK) and sleep logs on seven consecutive days, at baseline and during the fourth treatment week. Actigraphs recorded the amount of movement at one-minute epochs, 24 hours a day, and were worn on the nondominant wrist. Sleep logs were kept by the parents after having received careful instructions. Actigraphy data were converted into sleep parameters by a validated automatic scoring algorithm (Actiwatch, Cambridge Neurotechnology Ltd, Cambridge, UK) [113] using sleep log-derived lights out and rise time, and by subsequent manual verification on basis of sleep $\log$ data. In addition to the variables describing sleep per se, nonparametric variables describing the stability and fragmentation of the 24-hour rhythm in sleep and wakefulness were derived from the actigraphy data as described previously [217] (see Appendix III, for a definition of all sleep variables). The three pre-specified, primary sleep outcome parameters were: actigraphy-derived sleep onset and total time asleep, and sleep log-derived difficulty falling asleep. 
Dim light melatonin onset. Dim light melatonin onset (DLMO) is the clock time at which the endogenous melatonin level starts to rise in the subjective evening, and is considered the most reliable phase marker of the biological clock thythm [108]. The DLMO was assessed twice: at baseline and on the first evening of the fourth treatment week. Hourly samples of saliva from 18:00 to 22:00 h (age 6-7 years), or 19:00 to 23:00 h (age 7-12 years) were obtained by chewing on a cotton plug for one minute (Salivetten: Sarstedt Nümbrecht, Germany). No medication was taken at the evening of the salivary sample collection. To prevent suppression of melatonin secretion by bright light, curtains needed to be closed, and only one dim light was allowed during the entire measurement period. DLMO was defined as the linearly interpolated time at which the melatonin concentration first reached a level of $4 \mathrm{pg} / \mathrm{ml}$. Melatonin concentrations were obtained with radioimmunoassay as described previously [142].

Problem behavior. The primary pre-specified outcome parameter was the averaged grade $(1=$ very severe; $10=$ none $)$ on three, individually-defined, most serious and common core problems of the child, given by the parents as well as the teacher. Emotional and behavioral problems were assessed with the Child Behavior Checklist (CBCL, parent form) [6] and the Teacher's Report Form (TRF, teacher form) [5]. Both consisted of 120 items for which the respondent had to indicate on a three-point response scale $(0=$ not true; $1=$ somewhat or sometimes true; $2=$ very true or often true) whether an item held true for the child in recent weeks. Both questionnaires yielded a total score and eight syndrome scale-scores (high scores indicated more emotional and behavioral problems).

Cognitive performance. Interference control is the ability to ignore information that is linked to an inappropriate response tendency and has been shown impaired in ADHD [53;114]. The Eriksen task is a computerized choice reaction time task of interference control. Subjects had to respond on the direction of a target arrow, while, occasionally, a conflict occurred between this designated response and a competing response tendency elicited by incongruent flanking arrows. Subjects were instructed to focus on the target element and to ignore the flankers. Compared with the congruent and neutral flankers, the conflicting information produced by the incongruent flankers has been reported to lead to reduced error incidence $\left(\mathrm{EI}_{\Delta}, \%\right.$ ) ([number of errors on incongruent stimuli / total number of incongruent stimuli] - [number of errors on congruent stimuli / total number of congruent stimuli] * 100\%), and delayed reaction times ( $\mathrm{RT}_{\Delta}$, milliseconds) (RT on incongruent stimuli - RT on congruent stimuli). We administered six blocks of 60 
stimuli, of which one was a practice block. The experimental methodology has been described in detail elsewhere [172].

Sustained attention is the capacity to maintain focus of attention over time and was shown impaired in ADHD, with improvement after stimulant treatment (review see [171]). The Sustained Attention Dots Task (SADT) is a computerized visual task from the Amsterdam Neuropsychological Tasks [63] in which subjects were presented a continuous and consecutive series of 300 'target' $(33 \%)$, and 'nontarget' (67\%) asymmetric dot configurations, in a pseudo random fashion. They were instructed to push a "yes" button when a 'target' dot pattern was presented, and a "no" button when they saw a 'nontarget' dot pattern, then the next stimulus was presented immediately after the subject pressed a button (i.e. self-paced). The task parameters were accuracy (\%) ([number of misses + false alarms] / [total number of stimuli] *100\%), and task completion time (sec).

Quality of life. Health-related quality of life was assessed using the TNO-AZL Questionnaire for Children's Health-Related Quality of Life, Parent Form (TACQOL-P) [220;221]. The TACQOL-P consisted of 63 items on which the respondent rated on a three-point Likert scale whether or not a specific problem occurred or feeling had been present in recent weeks. A total sum score was calculated (maximal total sum score of 224 indicating the highest quality of life) as well as scores on seven sub-domains.

Adverse events. Parents were instructed to report all adverse events in an unstructured face-to-face interview $(\mathrm{KBH})$ three weeks after the start with study treatment. A follow-up was conducted in children who participated more than two years ago, using a self-constructed, structured questionnaire with items on various treatment aspects and adverse effects.

\section{Data analysis}

A total of 107 patients were randomly assigned to receive either melatonin or placebo (see Appendix IV for calculations of the required sample size). Randomization was performed by a hospital pharmacist who was not connected to the study, and occurred in blocks of four to keep the number of patients in each treatment group closely balanced at all times. The following stratification criteria were used: 1) presence of psychiatric co-morbidity (disruptive behavior disorder $[\mathrm{n}=59]$; anxiety disorder $[\mathrm{n}=16]$; depressive disorder $[\mathrm{n}=1]) ; 2)$ age category (6-9 years [ $\mathrm{n}=67]$; $10-12$ years $[\mathrm{n}=39]$ ); and 3 ) body weight category $(<40 \mathrm{~kg}[\mathrm{n}=88]$; $\geq 40 \mathrm{~kg}[\mathrm{n}=17])$. All investigators and participants were unaware of the treatment 
allocation. The code was broken after all children had completed their treatment and data were recorded in the database (October 2005). Comparisons of demographic and clinical characteristics between the two treatment groups was conducted with the use of an independent samples T-test for continuous variables with a normal distribution, with a Mann-Whitney $U$ test when the distribution of the continuous dependent variable was not normal, and with a Pearson chi-square test for discrete categorical variables. We calculated between-group differences in mean daylength (h) and mean rate of change in daylenght (hr/week) to control for potential confounding from these factors.

Analyses of pre- to post treatment changes within each treatment group were conducted using paired-samples T-tests. Analyses on the difference in pre-to post treatment changes between the treatment groups was conducted using General Linear Model (GLM) repeated measures analysis of variance with treatment group as between-subjects factor and time as within-subjects factor. Partial eta-squared effect sizes (PES) were computed which represent the proportion of the total variation that is attributable to the treatment effect (small if 0.01 , medium if 0.06 , and large above 0.14) [46]. A between-group comparison of pre- to post treatment changes in categorical variables was analyzed with Pearson chi-square tests. Included in the efficacy analyses were all patients who had been randomly assigned to, and had received treatment (intention-to-treat). Relation of pre- to post treatment changes and variables of interest were analyzed with linear regression analysis. Between-group differences in adverse events were analyzed with Fisher's exact test.

Significance was set at $p \leq 0.05$ (two-sided). To compensate for the increased probability of a type I error, Bonferroni correction of $p$ values was applied when multiple tests were conducted on questionnaire subscales (CBCL, TRF, TACQOL) calculated by multiplying the resulting $p$ values by the number of outcomes being tested (corrections are indicated). Statistical analyses were conducted using the Statistical Package for the Social Sciences, Version 12.0.1. (SPSS, Inc., Chicago, IL, USA).

\section{Results}

\section{Baseline demographic and clinical characteristics}

Of 107 patients who were randomly assigned to receive melatonin ( $n=53$ ) or placebo $(n=52), 105$ patients actually received treatment and completed the study, whereas two were withdrawn because, shortly after assignment, they started with other therapy without permission (Figure 1). At baseline, there were no significant between-group differences in demographic variables or clinical characteristics 
(Table 1). Furthermore, we found no differences between the two treatment groups in mean daylength $(\mathrm{h})$ or mean rate of change in daylenght (hr/week).

\section{Efficacy Measures}

Sleep. Pre- and post treatment actigraphy data were obtained from 80/105 participants, and sleep log data from $91 / 105$ participants. Missing data were due to technical problems, loss by participants, and not keeping to instructions. In the melatonin group, the mean ( \pm SD) actigraphic estimate of sleep onset advanced by $-0: 27 \pm 0: 48 \mathrm{~h}$, whereas in the placebo group there was a delay of $+0: 10 \pm 0: 37 \mathrm{~h}$ $(p<0.0001 ; \mathrm{PES}=0.162$ ) (Table 2). After melatonin treatment, 20 children showed an advance of sleep onset of more than 30 minutes (out of 41 participants from whom data were obtained [48.8\%]), while this was $5 / 39(12.8 \%)$ after placebo $\left(\chi^{2}=12.03 ; p=0.001\right)$.

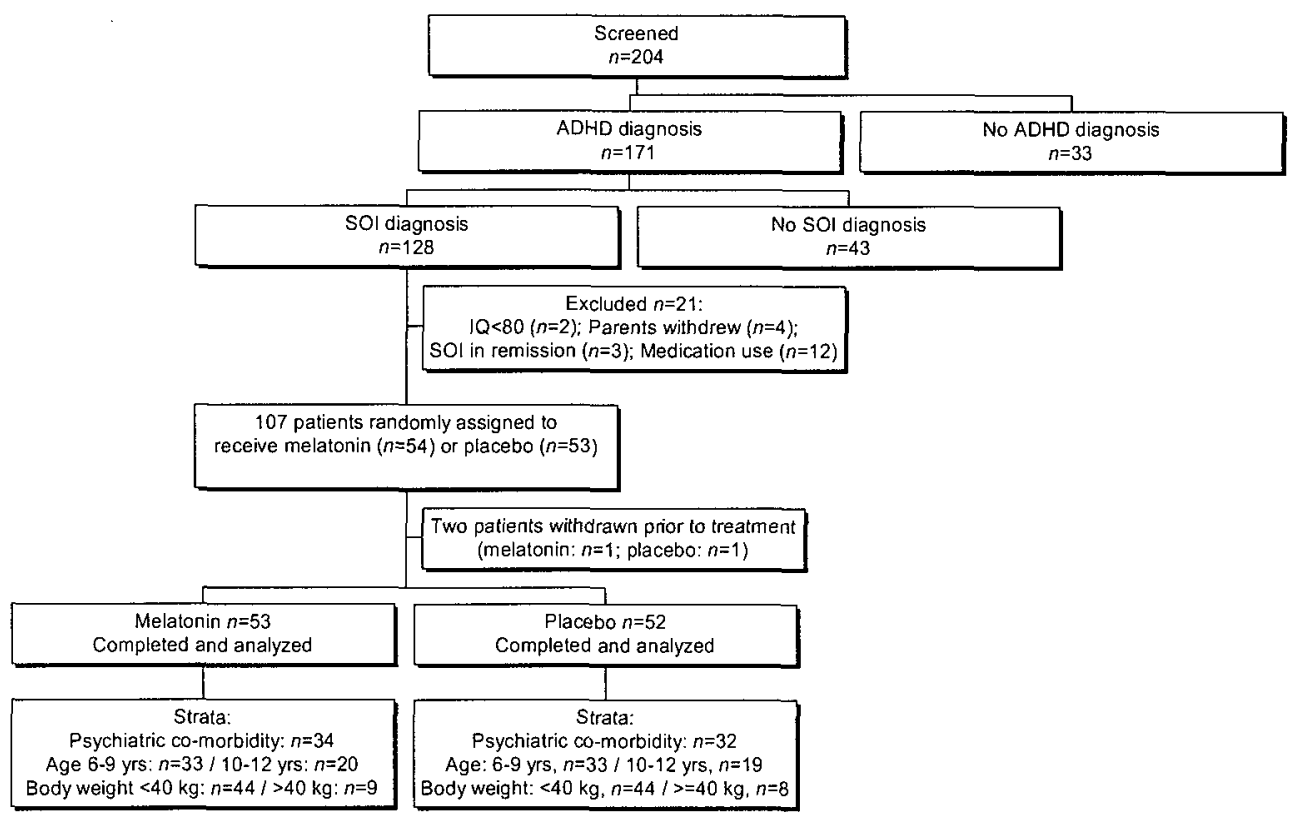

Figure 1. Participant flow diagram. SOI: chronic sleep onset insomnia. 
The actigraphic estimate of sleep latency decreased by $-21.30 \pm 32.96$ min with melatonin, and increased by $+3.04 \pm 31.74 \mathrm{~min}$ with placebo $(p=0.001$; PES $=0.127)$. There was an increase in mean total time asleep of $+19.82 \pm 61.90$ min with melatonin and a decrease of $-13.58 \pm 50.60$ min with placebo $(p=0.01$; PES $=0.082$ ). Sleep efficiency increased by $+2.60 \pm 8.92 \%$ with melatonin, whereas it decreased by $-2.11 \pm 7.12 \%$ with placebo $(p=0.01 ; \mathrm{PES}=0.08)$. There were no statistically significant group-differences in pre- to post changes of lights out, wake up time, time spent moving during sleep, or interdaily stability and intradaily variability of sleep-wake rhythm.

Table 1. Demographic and clinical patient characteristics.

\begin{tabular}{|c|c|c|c|}
\hline & $\begin{array}{l}\text { Melatonin } \\
(n=53)\end{array}$ & $\begin{array}{l}\text { Placebo } \\
(n=52)\end{array}$ & $p$ Value \\
\hline Age, mean $\pm S D(y r s)$ & $9.12 \pm 2.27$ & $9.31 \pm 1.83$ & $0.637^{\ddagger}$ \\
\hline Body weight, mean $\pm \mathrm{SD}(\mathrm{kg})$ & $31.8 \pm 10.3$ & $32.7 \pm 9.7$ & $0.671^{\ddagger}$ \\
\hline Male, No. (\%) & $35(66.0)$ & $43(82.7)$ & $0.051^{11}$ \\
\hline \multicolumn{4}{|l|}{ ADHD subtype, No. (\%) } \\
\hline ADHD-C & $41(77.4)$ & $36(69.2)$ & $0.335^{\pi}$ \\
\hline ADHD-I & $9(17.0)$ & $13(25.0)$ & $0.311^{\sharp}$ \\
\hline ADHD-HI & $2(3.8)$ & $2(3.8)$ & $0.984^{\pi}$ \\
\hline \multicolumn{4}{|l|}{ Psychiatric co-morbidity, No. (\%) } \\
\hline Distuptive behavioral disorder & $31(58.5)$ & $28(53.8)$ & $0.712^{\sharp}$ \\
\hline Anxiety disorder & $7(13.2)$ & $9(17.3)$ & $0.530^{\natural}$ \\
\hline Depressive disorder & $1(1.9)$ & $0(0)$ & $0.324^{9}$ \\
\hline Insomnia score (SDQ) & $2.57 \pm 0.49$ & $2.63 \pm 0.62$ & $0.692^{\wedge}$ \\
\hline PLMS/RLS score (SDQ) & $1.59 \pm 0.66$ & $1.55 \pm 0.58$ & $0.841^{\wedge}$ \\
\hline OSAS score (SDQ) & $1.20 \pm 0.27$ & $1.19 \pm 0.30$ & $0.806^{\mathbf{4}}$ \\
\hline Sleep hygiene score (CSHS) & $55.4 \pm 10.2$ & $57.2 \pm 10.2$ & $0.404^{\star}$ \\
\hline
\end{tabular}

ADHD-C: ADHD combined subtype; ADHD-I: ADHD inattentive subtype; ADHD-HI: ADHD hyperactive/impulsive subtype; SDQ: Dutch Sleep Disorders Questionnaire (scale $1=$ never to $5=$ =always)[199]; PLMS/RLS: period limb movement disorder/restless legs syndrome; OSAS: obstructive sleep apnea; CSHS: Children's Sleep Hygiene Scale (scale $25=\min$ to $150=\max$, higher values indicating worse sleep hygiene)[84].

${ }^{\ddagger}$ Independent samples T-test on the difference between melatonin and placebo.

" Pearson Chi-Square test on the difference between melatonin and placebo.

$\triangle$ Mann-Whitney U Test on the difference between melatonin and placebo. 
Nocturnal restlessness (L5) decreased by $-5.32 \pm 37.85$ arbitrary units $(11.9 \%$ of baseline) with melatonin, whereas it increased by $+14.54 \pm 40.75$ arbitrary units ( $40.4 \%$ of baseline) with placebo $(p=0.029$; PES $=0.062$ ). The mean score on sleep $\log$ item 'difficulty falling asleep', decreased by $-1.21 \pm 1.26$ points $(35.3 \%$ of baseline) with melatonin, and by $-0.14 \pm 0.75$ points ( $4.3 \%$ of baseline) with placebo $(p<0.0001$; PES $=0.213)$.

Dim light melatonin onset. Pre- and post treatment dim light melatonin onset values were obtained from $76 / 105$ participants. Missing values were due to an insufficient volume of collected saliva or not keeping to instructions.

Melatonin treated children showed an advance of dim light melatonin onset (DLMO) of $-0: 44 \pm 1: 07 \mathrm{~h}$, whereas there was a delay of $+0: 13 \pm 0: 59 \mathrm{~h}$ in the placebo treated children $(p<0.0001$; PES $=0.169)$. In the melatonin group, the preto post treatment change in sleep onset showed a significant linear relationship with pre-treatment DLMO ( $\mathrm{df}=1,36 ; \mathrm{R}=0.42 ; p=0.008)$, indicating that more delayed DLMO values at baseline were associated with stronger advances of sleep onset after melatonin treatment. This relationship was not present in placebo $(\mathrm{df}=1,35$; $\mathrm{R}=0.078 ; p=0.645$ ). In the melatonin group, the pre- to post treatment change in DLMO was not significantly related to the pre- to post treatment change in sleep onset $\left(\mathrm{df}=1,25 ; \mathrm{R}^{2}=0.30 ; p=0.124\right)$.

\section{Problem behavior.}

Core problems: Pre- and post treatment data on core problems were obtained from 86/105 parents, and 58/105 teachers. Missing data were due to loss of not keeping to instructions.

The mean baseline severity grade was $4.02 \pm 1.25$ in the melatonin group and $4.31 \pm 1.33$ in placebo, from a maximum score of 10 (i.e. no problems). The most frequently reported problems were easily getting angry (36.0\%); sleep onset problems (31.8\%); and attention problems (23.3\%). The mean severity grade of the three parent-defined core problems improved by $+0.74 \pm 0.94$ (18.4\% of baseline) with melatonin treatment, and by $+0.16 \pm 0.76$ (3.71\% of baseline) with placebo treatment $(p=0.002$; PES $=0.105$ ) (Table 3 ). After removing the core symptoms that were related to sleep (31.8\%), the group difference lost statistical significance.

The mean averaged grade of core problems reported by the teacher improved by $+0.68 \pm 0.93$ with melatonin, and by $+0.47 \pm 0.98$ with placebo $(p=0.414$ PES $=0.012$ ). None of the core problems reported by teachers were related to sleep. 
Table 2. Effects of melatonin and placebo treatment on dim light melatonin onset and objective and subjective measures of sleep in children with ADHD and chronic sleep onset insomnia.

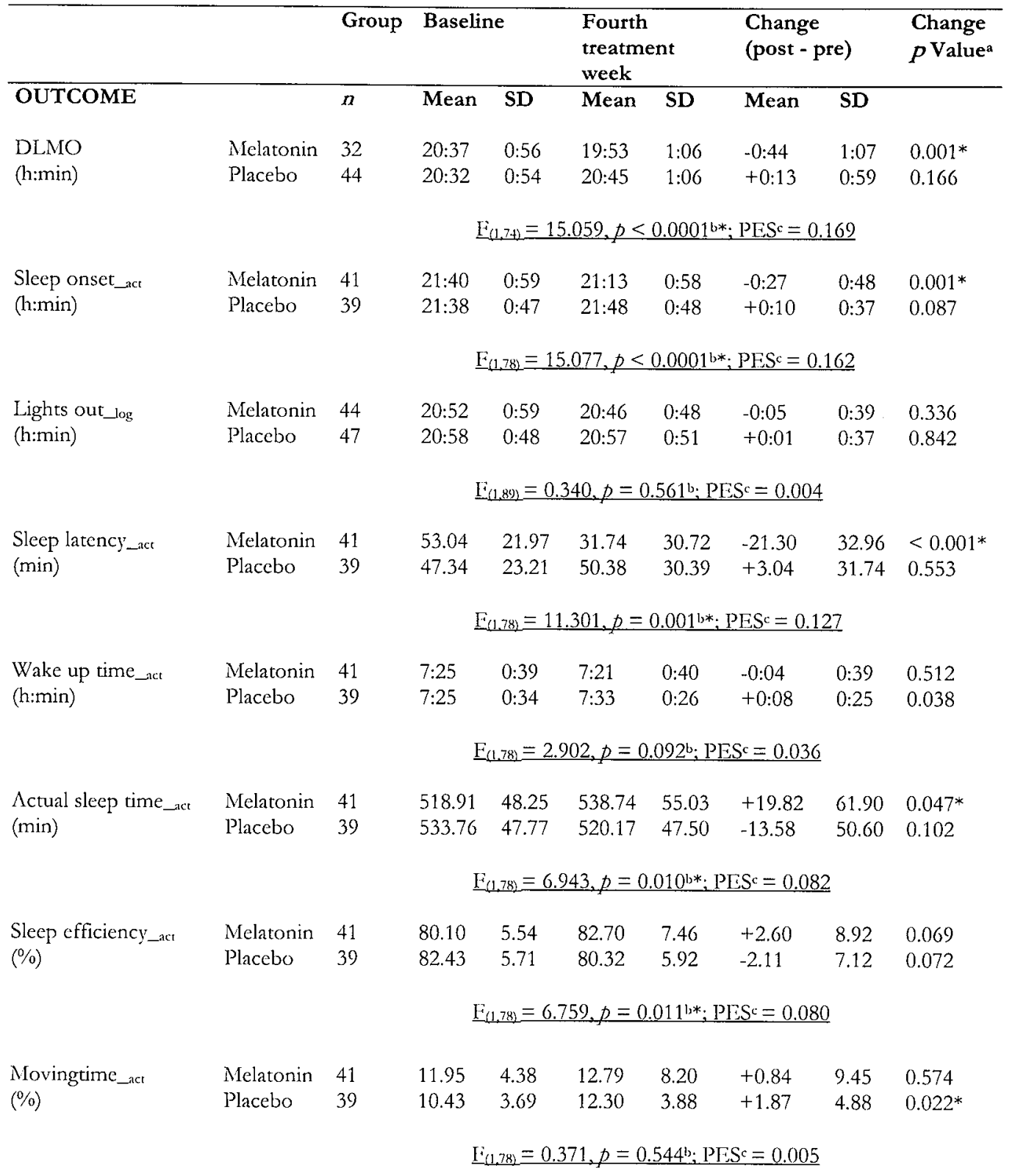


Table 2. Effects of melatonin and placebo treatment on dim light melatonin onset and objective and subjective measures of sleep in children with ADHD and chronic sleep onset insomnia (continued)

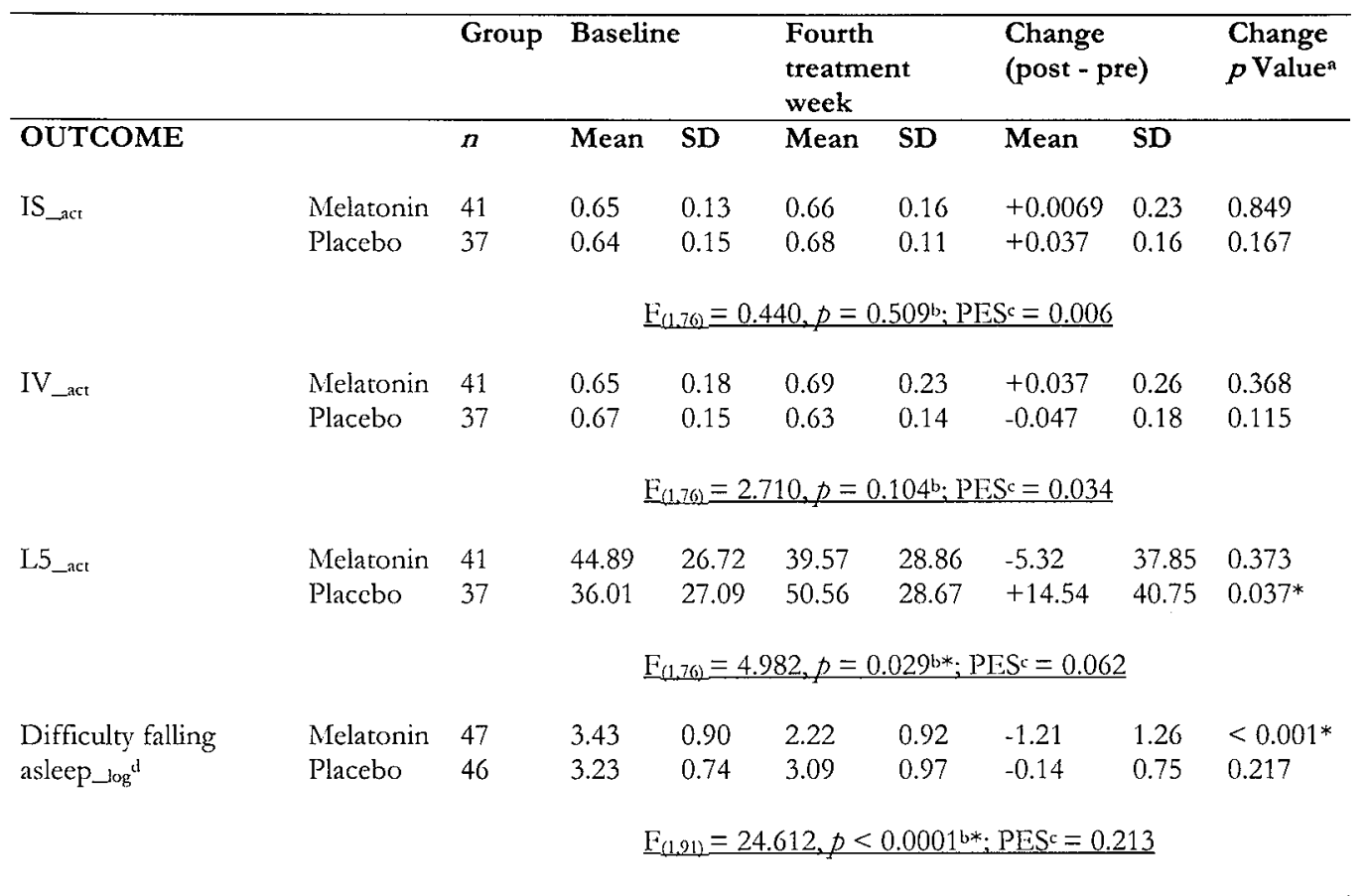

Note: $\mathrm{SD}=$ standard deviation; DLMO = salivary dim light melatonin onset; act = actigraphy data; $\log =$ sleep $\log$ data; IS = interdaily stability (range from 0 to 1 , higher values indicating more stable rhythms); IV = intradaily variability (range from 0 to 2, with higher values indicating more fragmented rhythms); L5 $=$ average activity level in least active 5 -h period.

* Statistically significant difference ( $p \leq 0.05$, two-sided).

a Paired-samples T-tests on mean within-group change from baseline-to-treatment score.

b Gencral linear model repeated measures test on group-difference in mean change from baseline-totreatment $(p \leq 0.05$, two-sided).

c Partial eta squared effect size (small if 0.01 , medium if 0.06 , and large above 0.14 ).

a Mcan difficulty falling asleep as reported by parents, averaged over 7 days, scale 1 (not difficult) to 5 (very difficult). 
Table 3. Effects of melatonin and placebo treatment on core problems, emotional/ behavioral problems, quality of life, and performance on tests of interference control and sustained attention in children with $\mathrm{ADHD}$ and chronic sleep onset insomnia.

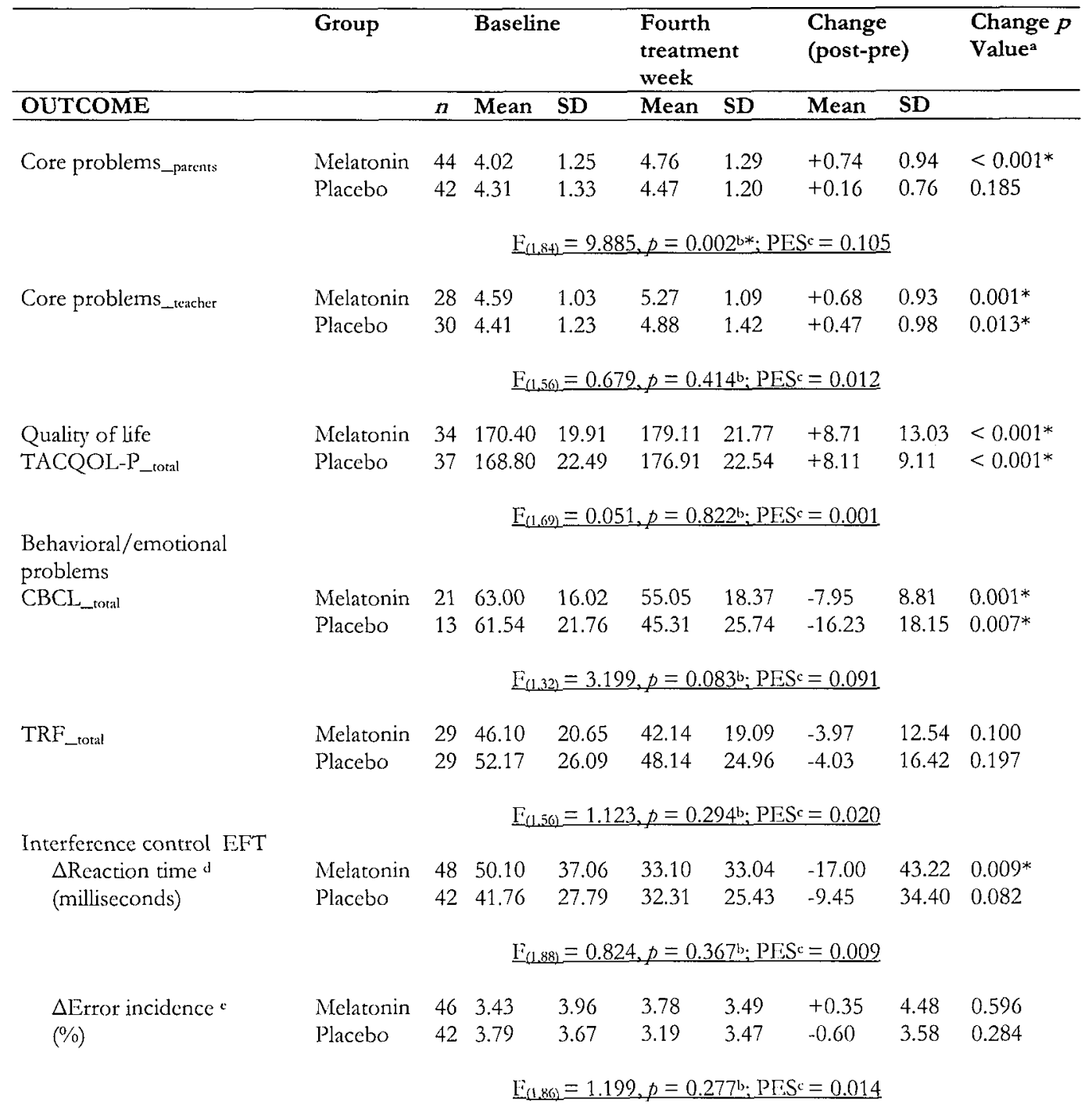

Sustained attention $\Lambda \mathrm{NT}_{-}$ SADT

Accuracy ${ }^{\mathrm{i}}$

$(\%)$

$\begin{array}{lllllllll}\text { Melatonin } & 44 & 12.55 & 7.60 & 12.23 & 7.21 & -0.32 & 4.52 & 0.643 \\ \text { Placebo } & 43 & 11.98 & 6.67 & 12.37 & 7.29 & +0.39 & 5.55 & 0.649\end{array}$

$E_{(1.85)}=0.424, p=0.517 \mathrm{~b} ; \mathrm{PES}=0.005$ 
Table 3. Effects of melatonin and placebo treatment on core problems, emotional/ behavioral problems, quality of life, and performance on tests of interference control and sustained attention in children with ADHD and chronic sleep onset insomnia (continued).

\begin{tabular}{llllll}
\hline Group & Baseline & $\begin{array}{l}\text { Fourth } \\
\text { treatment } \\
\text { week }\end{array}$ & $\begin{array}{l}\text { Change } \\
\text { (post-pre) }\end{array}$ & $\begin{array}{l}\text { Change } p \\
\text { Value }^{\mathrm{a}}\end{array}$ \\
\hline OUTCOME & $n$ Mean SD & Mean SD & Mean SD \\
\hline
\end{tabular}

Sustained attention ANT_ SADT

Task completion time (seconds)

$\begin{array}{lllllllll}\text { Melatonin } & 44 & 468.99 & 152.95 & 410.47 & 142.59 & -58.52 & 77.80 & <0.001^{*} \\ \text { Placebo } & 43 & 489.41 & 187.13 & 428.18 & 171.91 & -61.23 & 85.54 & <0.001^{*}\end{array}$

$\underline{F}_{(185)}=0.312, p=0.578^{\mathrm{b}} ; \operatorname{PESc}=0.004$

$\mathrm{SD}=$ standard deviation; TACQOL-P = TNO-AZL Questionnaire for Children's Health-Related Quality of Life, Parent Form[221]; Parent =as reported by parents; Teacher = as reported by tcacher; $\mathrm{CBCl}_{\text {_total }}$ $=$ Child Behavior Checklist/4-18 years[6], total score; TRF_toal $=$ Teacher's Report Form[5], total score; EFT $=$ Eriksen Flanker Task; ANT_SADT $=$ Sustained Attention Dots Task of the Amsterdam Neuropsychological Tasks (AN'T) [63].

* Statistically significant difference ( $\phi \leq 0.05$, two-sided).

a Paired-samples T-tests on mean within-group change from baseline-to-treatment score.

${ }^{b}$ Gencral linear model repeated measures test on group-difference in mean change from baseline-totreatment $(\not \leq 0.05$, two-sided).

c Partial eta squared effect size (small if 0.01 , medium if 0.06 , and large abovc 0.14 ).

d The mean difference in reaction time between incongruent and congruent trials, in millseconds.

' The mean difference in error incidence ((number of errors / total number of stimuli) $* 100 \%$ ) berween incongruent and congtuent trial.

${ }^{i}$ Mean accuracy (percentage of misses plus falls alarms relative to the total number of trials).

Behavioral and emotional symptoms: Pre- and post treatment data on behavioral and emotional symptoms were obtained from 34/105 parents, and 58/105 teachers. Missing data were due to loss by participants, not keeping to instructions, or partial completion of questionnaires.

The total score on CBCL decreased by $-7.95 \pm 8.81$ with melatonin, and by $16.23 \pm 18.15$ with placebo $(p=0.083$; PES $=0.091)$. The teacher score decreased by $-3.97 \pm 12.54$ with melatonin, and by $-4.03 \pm 16.42$ with placebo $(p=0.294$; PES $=0.020$ ). Analyses of the eight subscales of the CBCL and TRF indicated only a significantly smaller improvement on the aggressive behavior subscale of the CBCL with melatonin $(-0.62 \pm 3.44)$ as compared to placebo $(-6.21 \pm 6.86)$ 
$(\mathrm{F}[1,33]=10.218$, Bonferroni corrected $p=0.024)$. The pre- to post treatment on total CBCL and TRF scores were not related to pre- to post treatment changes in actigraphy-derived sleep onset, total time asleep, or sleep log-derived difficulty falling asleep.

Cognitive performance. Pre- and post treatment data on interference control were obtained from 90/105 participants, and on sustained attention from 87/105 participants. Missing data were due to due to technical problems or to refusal of children to perform the task.

Interference control: At baseline, mean reaction time $( \pm \mathrm{SD})$ on incongruent trials was significantly slower $(696.83 \pm 146.41 \mathrm{sec})$ than on congruent trials $(650.80 \pm 140.08$ sec), $(p<0.0001)$. Furthermore, error incidence on incongruent trials $(7.78 \pm 6.36$ $\%$ ) was significantly higher than on congruent trials $(4.18 \pm 4.17 \%),(p<0.0001)$. This indicates that there was a significant effect of congruence on interference control. Melatonin treatment significantly reduced $\Delta$ Reaction time (reaction time incongruent trials - reaction time congruent trials) of correct responses with -17.00 $\pm 43.22 \mathrm{~ms}$, indicating an improvement of interference control, however, the difference with the reduction of $-9.45 \pm 34.40 \mathrm{~ms}$ in placebo was not significant $(p=0.367$; PES $=0.009)$. Similarly, the group-difference in pre- to post treatment changes in mean $\Delta$ Error incidence (error incidence incongruent trials - error incidence congruent trials) was not significant ( $p=0.277$; PES $=0.014$ ).

Sustained attention: The mean task completion time decreased with $-58.52 \pm 77.80$ sec in melatonin, and with $-61.23 \pm 85.54 \mathrm{sec}$ in placebo $(\phi=0.578$; PES $=0.004)$. Accuracy decreased with $0.32 \pm 4.52 \%$ after melatonin treatment and increased with $0.39 \pm 5.55 \%$ after placebo $(p=0.517$; PES $=0.005)$.

Quality of life. Pre- and post treatment data on quality of life were obtained from $71 / 105$ parents. Missing data were due to loss by participants, not keeping to instructions, or partial completion of questionnaires. The mean baseline level of the total score on the TACQOL-P was $170.4 \pm 19.91$ in the melatonin group and $168.80 \pm 22.49$ in placebo (maximum score 224). The total TACQOL-P score improved by $+8.71 \pm 13.03$ ( $5.1 \%$ of baseline) with melatonin and by $+8.11 \pm 9.11$ ( $4.8 \%$ of baseline) with placebo $(p=0.822$; PES $=0.001)$. No Bonferroni-corrected significant group differences were found for pre-to post treatment changes on any of the seven subscales. There was no significant linear relationship of pre- to post treatment changes on the mean total TACQOL-P score with the pre- to post treatment changes in actigraphy-derived sleep onset, total time asleep, or sleep logderived difficulty falling asleep, in either treatment group. 
Table 4. Adverse events in children with ADHD and chronic sleep onset insomnia treated with melatonin or placebo.

\begin{tabular}{llll}
\hline Adverse event & $\begin{array}{l}\text { Melatonin } \\
(n=53)\end{array}$ & $\begin{array}{l}\text { Placebo } \\
(\boldsymbol{n}=52)\end{array}$ & $p$ Value \\
& No. $\%)^{*}$ & $0(0)$ & \\
\hline Headache & $3(5.7)$ & $0(0)$ & 0.243 \\
Hyperactivity & $3(5.7)$ & $0(0)$ & 0.243 \\
Dizziness & $2(3.8)$ & $0(0)$ & 0.495 \\
Abdominal pain & $2(3.8)$ & $0(0)$ & 0.495 \\
Nose bleeding & $1(1.9)$ & $0(0)$ & 1.000 \\
Itching lumps on the skin & $1(1.9)$ & $0(0)$ & 1.000 \\
Painful lumps on the skin & $1(1.9)$ & $0(0)$ & 1.000 \\
Diarrhea & $1(1.9)$ & $0(0)$ & 1.000 \\
Decrease of mood & $1(1.9)$ & $0(0)$ & 1.000 \\
Maintenance insomnia & $1(1.9)$ & & 1.000 \\
\hline * Patients could report more than one event. & & \\
IFisher's exact test of group differences. &
\end{tabular}

Adverse events. There was no significant difference in number of adverse events between the melatonin and placebo treated groups (Table 4), nor between the $3 \mathrm{mg}$ $(8 / 44)$ and $6 \mathrm{mg}(2 / 9)$ treated group (Fisher's Exact test: $p=1.00$ ). Five patients showed one adverse event, four patients showed two adverse events, and one showed three adverse events There were no discontinuations or withdrawals due to adverse events, and none of the adverse events required other treatment.

Follow-up at two years after participation yielded $24 / 26$ completed questionnaires (two families were untraceable): 19/24 still used melatonin (mean dose ( \pm SD) $4.39 \pm 1.95 \mathrm{mg}$ taken at 19:42 $\pm 0.50 \mathrm{~h}$ ), one used it occasionally, and four stopped after a mean $( \pm$ SD) period of $17.25 \pm 3.30$ months (reasons: remission $(n=3)$, dizziness and drowsiness $(n=1))$. A number of $7 / 24$ parents reported one or more of the following adverse events: bedwetting ( $n=2)$; abnormal feces ( $n=2)$; drowsiness $(n=2)$; dizziness $(n=1)$; awakening at night and not able falling asleep again $(n=1)$; skin pigment changes $(n=1)$; decreased $\operatorname{mood}(n=1)$.

\section{Discussion}

This is the first randomized, placebo-controlled trial to investigate benefits and harms of melatonin in medication-free children with diagnosed ADHD and 
chronic sleep onset insomnia. Melatonin improved objective sleep onset and sleep duration, reduced subjective difficulty falling asleep, and induced advances of sleep onset of more than 30 minutes in about half of the melatonin treated children. However, the findings did not support our initial hypothesis that melatonin treatment improved problem behavior, cognitive performance, and quality of life. While parents reported an improvement in severity of individually-defined core problems, this was mainly due to an amelioration of problems related to sleep. Melatonin use was not associated with significant adverse events, which corresponds with previous randomized clinical trials in children with sleep disorders [65;98-100;131;151;190;191].

Melatonin advanced actigraphically estimated sleep onset $(-0: 27 \mathrm{~h})$ and salivary dim light melatonin onset $(-0: 44 \mathrm{~h})$ to 'normal' values found previously in children with ADHD without insomnia [213], or healthy children [212]. However, current phase shifts were less robust than found previously in insomniac children $(-0: 42 \mathrm{~h}$, and $-1: 12 \mathrm{~h}$ respectively) [212]. A possible explanation is that the baseline values of sleep onset and dim light melatonin onset were more delayed in the previous trials, which predicts more robust phase advances of melatonin administered in the early evening, as was shown previously $[120 ; 212]$, as well as in the present study. Although these results indicate that melatonin induced a synchronizing effect on the circadian clock (chronobiotic effect) we did not find a positive relationship between the melatonin-induced change in dim light melatonin onset and the change in sleep onset. This is not in line with what one should expect if 'Zeitgeber' were the only mechanism, therefore, other working mechanisms might have played a role such as a hypnotic effect related to changes in temperature gradients $[61 ; 96 ; 215 ; 216]$, or a possible influence at the GABA chloride channel complex $[180]$.

In spite of improvements in sleep, melatonin demonstrated no effect on behavior, cognitive performance, and quality of life in the current sample of medication-free children with ADHD. Possibly, behavioral disturbances were only minimally related to insomnia, although sleep problems and sleep deprivation in children are typically associated with behavioral disturbances [58;181;194]. In contrast to the present results, one previous study showed that melatonin treatment of childhood insomnia induced improvements in health status [191]. One other study did not find an effect of melatonin on sustained attention in children with insomnia [190]. A possible explanation is that the improvement of sleep in the current study was just not large enough to induce improvements in behavior, cognitive performance, and quality of life. Furthermore, such effects may have required longet treatment duration or may have been masked by large ADHD- 
related performance decrements. Of note, the negative placebo effect we found on the objective sleep parameters is in line with previous findings in insomniac children [190;212]. However, in adults with primary insomnia one usually finds positive placebo effects [162]. This discrepancy is an interesting phenomenon for which we do not have a clear explanation.

We used a pharmacological dose of 3 or $6 \mathrm{mg}$, dependent on body weight, whereas previous studies in children used pharmacological doses of 2 to $12 \mathrm{mg}$, which were found safe and effective [98-100;131;190;191]. In normal as well as insomniac adults, lower doses of melatonin at physiological levels $(0.1,0.3,1.0 \mathrm{mg})$ showed sleep-promoting effects [10;237]. Further studies are needed to evaluate whether physiological doses of melatonin are efficacious in insomniac children as well.

Strengths of the present study were the relatively large sample size and the application of rigorous diagnostic methods and strict diagnostic criteria for ADHD as well as chronic sleep onset insomnia. All patients were medication-free which excludes possible influences of medication on melatonin efficacy. Furthermore, we used objective as well as subjective measures of sleep, behavior, cognitive performance, and quality of life, and conducted a systematic two-year follow up of adverse events.

Nevertheless, several limitations of the present study need consideration. We used actigraphy as it is a well-validated instrument to evaluate sleep-wake rhythm in children [125]. Furthermore, it has the advantage over gold standard polysomnography that it can record over much longer periods. However, its consequence was that we were not able to objectively evaluate the presence of sleep-disturbing disorders such as periodic limb movement disorder or sleepdisordered breathing. Nevertheless, we found that reports of such symptoms were rare and equally distributed over the two treatment groups, which reduced the chance of spurious results. A further limitation with respect to missing polysomnography measurements was that we were not able to evaluate effects of melatonin on sleep stages, cycles, and their interrelationships, however, previous studies found that such effects were either not present or not clinically relevant [10;97]. Another limitation was the considerable number of missing data on some outcome measures of behavior and quality of life, which was mainly due to an inadequate completion of questionnaires. The fact that this occurred equally in both treatment groups minimizes the chance that a reporter bias may have distorted the data. However, the increased number of missing data might have led to a reduction in statistical power and, therefore, a higher chance on false negative results. 
The present findings are likely generalizable to other ADHD populations since we recruited from heterogeneous health care settings, included all ADHDsubtypes, and employed diagnostic methods according to widely used psychiatric classification systems and practice guidelines. However, two issues with regard to the generalizability need consideration. Firstly, our findings may not pertain to stimulant-treated ADHD patients since evidence suggests that stimulant treatment can exert deteriorating effects on sleep [7;193]. One open-label study in stimulanttreated ADHD children showed robust advances of sleep onset with a median of $135 \mathrm{~min}$, while there were no significant adverse events [203]. The combined use of ADHD treatment with melatonin needs further investigation in randomized placebo-controlled trials. Secondly, since international consensus criteria on childhood insomnia were not available, we used criteria of chronic sleep onset insomnia based on a Dutch child population, which were conform or even more strict than most recent consensus criteria for adult insomnia [11;69]. However, it remains unknown whether the present results also pertain to children in other cultures.

Although there is mounting evidence that the use of melatonin in children is safe in the short term, it needs to be emphasized that systematic studies on possible long-term effects, such as on the gonadotropic system and onset of puberty [128;168;188] have not been conducted [17]. Furthermore, in children with epilepsy, melatonin has shown pro-convulsant properties [186], although anticonvulsant effects were found as well [159]. The results of our two-year follow up cautiously suggest that long term treatment with melatonin was safe. However, a careful use of melatonin in children is required until a thorough evaluation of longterm health effects has been conducted in future studies.

In summary, the results of this study indicate a positive balance of benefits and risks for the use of melatonin treatment in medication-free children with ADHD and chronic sleep onset insomnia. It can be applied for the treatment of persistent and severe insomnia, but not for the treatment of ADHD-related impairments of behavior, cognitive performance, and quality of life. Melatonin should be used only when the insomnia imposes a considerable burden on the individual child, if possible after amelioration of possible underlying extrinsic factors(s), and preferably in those children demonstrating a delayed onset of endogenous melatonin thythm. Replication of the present study is needed including a careful evaluation of its use over the long term, such as on adverse effects, needed treatment duration, and occurrence of relapses. 


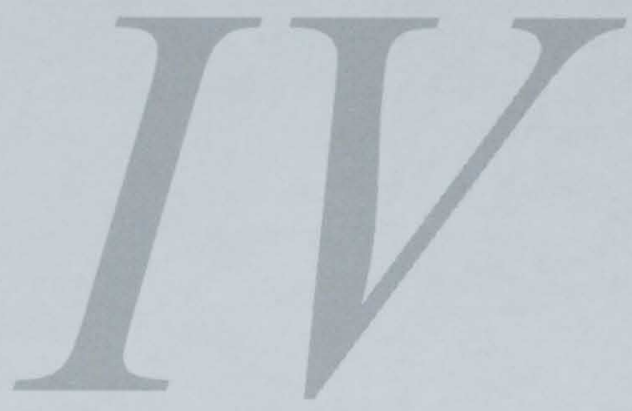

General discussion 

General discussion and implications for health care and future research 
The preceding chapters have shown new insights as to the etiology and treatment of chronic sleep onset insomnia in children with ADHD. In this section, I will comment on the main issues and relate the findings to those found in the literature. Where appropriate, limitations and possible implications for health care and future research are discussed.

This thesis provided evidence that chronic sleep onset insomnia in ADHD should be considered a circadian rhythm sleep disorder with a likely etiological role of a biological clock disturbance. This could be inferred from a rigid misalignment between the patient's children's sleep-wake pattern and the desired pattern, accompanied with a delay in the most reliable phase marker of the biological clock rhythm, i.e. dim light melatonin onset, while the maintenance of sleep was normal (Chapter five). The delay in circadian rhythms was unlikely to be induced by later bedtimes, since we found no difference in lights-out compared to children with ADHD without insomnia. Furthermore, the influence of other factors was controlled for, such as the presence of comorbid psychiatric or neurological disorders, use of medication, and differences in demographic characteristics. Notwithstanding, we prefer to continue using the term chronic sleep onset insomnia since circadian rhythm sleep disorders are still poorly recognized and inadequately defined in children. An issue that merits consideration and further research is that characteristics of a circadian rhythm sleep disorder may not be specific to insomniac children with $\mathrm{ADHD}$, but may pertain to the wider population of child insomniacs. It was shown in one of our studies that elementary school-aged children with chronic sleep onset insomnia who were otherwise healthy, also showed a delayed dim light melatonin onset and a delayed sleep-wake rhythm (see Chapter seven).

Studies have shown that melatonin phase estimates of the biological clock rhythm showed a greater stability than other markers, probably due to a reduced chance of masking by other factors, and the fact that melatonin levels are under a relatively direct control of the biological clock [24;108]. Hence, melatonin phase estimates seem to remain stable even when the period between consecutive assessments of individual subjects was nine months [24]. Nevertheless, an important feature of the biological clock is to adapt to the alternating environment. In healthy human beings, the endogenous melatonin rhythm shows moderately rapid adjustments to changes in the environment [233]. In medication-free children with ADHD and chronic sleep onset insomnia, we found a persistent and rigid delay of the internal circadian phase with respect to the desired sleep-wake 
schedule. It remains unknown which factors are involved in the pathophysiology of this condition. Possibly, the adaptive properties of the circadian pacemaker may be impaired, leading to an inability to advance the sleep phase to earlier hours by enforcing conventional sleep and wake times. Furthermore, the intrinsic circadian period length may be longer than 24 hours similar to that in the non-24-hour sleepwake syndrome. Further studies are needed to evaluate these issues using natural changes in the environment such as in weekends or vacations, or manipulations in sleep laboratories with constant routine and forced desynchrony paradigms.

We found a markedly higher frequency of a history of childhood chronic sleep onset insomnia in first-degree relatives of insomniac children with ADHD compared to ADHD controls without insomnia (Chapter three), which is supported by similar recent findings of a familial aggregation in adult insomniacs [60]. In the majority of medication-free children with $\mathrm{ADHD}$, the start of sleep onset insomnia occurred before the age of three years (Chapter five). Altogether, these findings suggest that predisposing factors with a possible genetic nature play an etiological role. Our hypothesis that a VNTR polymorphism of the PER3 gene of the biological clock was an important genetic factor in the predisposition to chronic sleep onset insomnia in ADHD was not supported (Chapter 6). However, a limitation in this study was the relatively small sample size, which has restrained the statistical power and, consequently, induced a considerable chance of false negative findings. Hence, replication of this study is needed incorporating not only an analysis of the PER3 VNTR polymorphism, but also other components of the circadian pacemaker such as the recently discovered N408 allele in Casein Kinase I Epsilon, which phosphorylates clock proteins and plays a potential protective role in the development of circadian thythm sleep disorders [201].

Sleep hygiene covers all the habits and environmental conditions that can determine the quality of a child's sleep. Bad parenting can lead to inadequate sleep hygiene and might, therefore, play a role in the acquirement or maintenance of chronic sleep onset insomnia. In this thesis, we did not find a significant difference in sleep hygiene between children with ADHD and chronic sleep onset insomnia and ADHD controls without insomnia. In addition, sleep hygiene levels in both groups were similar to levels found previously in the general child population of the United States [84]. These findings indicate a limited role of sleep hygiene in the etiology of chronic sleep onset insomnia in medication-free children with ADHD. Notwithstanding, it needs to be emphasized that screening of possible sleep hygiene inadequacy remains the first step to be taken in the clinical management of 
chronic sleep onset insomnia in children with ADHD, before other often more expensive and invasive treatment strategies will be employed.

In the individual child, the etiology of insomnia is often a combination of different predisposing, precipitating, and perpetuating factors. Predisposing factors determine a vulnerability to more direct causes (precipitating factors, see later in this section) that act close to the onset of the illness. As for insomnia in children with $\mathrm{ADHD}$, we found a likely predisposing role of a biological clock disturbance that was possibly related to genetic factors (as discussed above). Furthermore, this thesis showed that a concomitant depressive disorder may put a child with ADHD at risk to develop chronic sleep onset insomnia (Chapter three). An explanation may be that rumination, negative thoughts and worry are associated with increased physical and mental arousal that is not compatible with sleep onset [59]. However, the cross-sectional retrospective design of our study did not allow for an inference of causality. Recent findings in adults suggested that insomnia can also be a precursor or risk factor to the onset of major depressive disorder [73]. So, it remains unknown whether in our study sample the insomnia was a precursor, symptom, or residual symptom of the depression [109]. Although predisposing factors may put a child at risk to develop insomnia, distinct precipitating factors were involved that occurred shortly before onset of the insomnia and most likely triggered the insomnia. Psychostimulant treatment for ADHD precipitated chronic sleep onset insomnia in twenty percent, and exacerbated existing insomnia in a further fifteen percent (Chapter three). These findings corroborated previous findings of stimulant-induced disturbances of sleep [7;193], although contradictory data have also been found [150] (Chapter two). A recent study showed that the most common precipitating factor of insomnia in adolescents and adults was stress at school or work [23]. Given the frequent problems that children with ADHD encounter at school, this may be an interesting topic for further investigation. Typically, after insomnia has progressed into a chronic disorder in adults, it is sustained by perpetuating factors such as staying in bed longer because one has not slept well, learning to worry, and desperately trying to fall asleep [87]. We did not evaluate such perpetuating factors in this thesis, and no other studies to date have investigated whether these factors play a role in children.

It is the responsibility of the practicing clinician to verify the major etiological factor(s) for the individual patient's complaints, and classify it accordingly following the diagnostic classification system for sleep disorders (ICSD) [11]. The results of the preceding chapters suggest that important classifications for the differential 
diagnosis of children with ADHD and chronic sleep onset insomnia are the circadian rhythm sleep disorder (e.g. delayed sleep phase syndrome), stimulantdependent sleep disorder, and sleep disorder associated with mental disorders. But also other classifications of the ICSD might pertain to this population such as the limit-setting disorder, sleep-onset association disorder, idiopathic insomnia, or inadequate sleep hygiene. The chapters five and eight demonstrate that currently no international consensus exists on diagnostic criteria of chronic sleep onset insomnia or circadian thythm sleep disorders in children. Effort should be made to achieve progress towards establishing clinical guidelines or practice parameters for such sleep disorders in children.

The retrospective cross-sectional study in Chapter two revealed that the point prevalence of sleep onset insomnia in the clinical population of children with ADHD was $28.6 \%$, which was very similar to the $27.8 \%$ and $32.1 \%$ found previously in medication-free psychiatric and community ADHD samples [50;193], and was markedly higher than the estimated prevalence in the general child population of $8-15 \%[27 ; 135 ; 156]$. This indicated the presence of a probable association between ADHD and chronic sleep onset insomnia. However, as the review in Chapter two pointed out, while retrospective studies using rating scales generally found a link between $\mathrm{ADHD}$ and chronic sleep onset insomnia, prospective studies of sleep continuity measures failed to find such a relationship. An explanation for this discrepancy may lie in the classic feature of insomnia that it shows a strong night-to-night variability, with very poor nights alternating with normal or moderately poor nights [209]. Hence, a single day measurement of sleep may fail to observe the insomnia problems, whereas calculating average values of multiple-day measurements may strongly reduce the power to reveal significant differences with non-insomniac control groups. For that reason, future prospective studies should be conducted employing an analysis of the frequency and severity of poor nights of sleep in children with ADHD and controls. Currently, we are performing a study addressing this issue in medication-free children with ADHD and chronic sleep onset insomnia, by an evaluation of the concordance of actigraphy, polysomnography and log measurements of sleep.

Melatonin, administered at the right time, is considered an appropriate treatment strategy for circadian rhythm sleep disorders [56;143;144], and has previously been found effective and safe in children with childhood chronic sleep onset insomnia [190;191;212]. As discussed above, medication-free children with ADHD and chronic sleep onset insomnia showed characteristics of a circadian rhythm sleep 
disorder, such as a significantly delayed sleep-wake rhythm and dim light melatonin onset in the absence of sleep maintenance disturbances [213]. In chapter nine, a randomized, placebo-controlled trial is described in which we investigated the benefits and harms of a pharmacological dose of melatonin in this clinical population. To our knowledge, this is the first time that such an endeavor has been undertaken. Earlier studies have already established that treatment of sleep-related disorders other than insomnia seem to improve daytime functioning in children with ADHD [8;79;104;225]. This outcome was expected as well in our study, which could have important consequences for ADHD treatment strategies in health care settings. The results showed that melatonin improved objective sleep onset and sleep duration, as estimated by actigraphy, reduced subjective difficulty falling asleep, and induced advances of sleep onset of more than 30 minutes in about half of the treated children. However, the hypothesis that melatonin treatment improved behavior, cognitive performance, and quality of life was not supported (discussed later in this chapter). While parents reported improvements in core problems of daily life, this was mainly due to an amelioration of problems related to sleep.

In children with ADHD and chronic sleep onset insomnia, melatonin treatment advanced dim light melatonin onset to values within the 'normal' range, i.e. values that were found in children with ADHD without chronic sleep onset insomnia and in healthy child controls (Chapter seven). This finding supports the notion that melatonin induces a chronobiotic effect, that is, it helps to reset circadian thythms. However, we did not find a positive relationship between melatonin-induced changes in dim light melatonin onset and changes in sleep onset, which is not in line with what one would expect if 'Zeitgeber' were the only component involved in the working mechanism of melatonin. Hence, other components might have played a role such as a hypnotic effect emanating from changes in temperature gradients $[61 ; 96 ; 215 ; 216]$, or a possible influence at the GABA chloride channel complex [180].

Melatonin shifts circadian thythms according to a phase-response curve in which the magnitude of the phase advance depends on the timing of administration within the phase-advance zone (from six hours after, to six hours before the subjective beginning of the day). In accordance with these findings we demonstrated that when administering melatonin in the evening (around six or seven o'clock), the phase-advance in sleep-wake rhythm that it induced was more robust when dim light melatonin onset was initially more delayed (Chapter seven). 
In other words, the efficacy of melatonin treatment to advance sleep-wake thythm could be predicted from pre-treatment dim light melatonin onset. Therefore, given this phase-response relationship of melatonin, the decision on at what time melatonin should be administered in the clinical situation should be based on the patient's individual circadian phase estimate. In adults, maximal phase advances of sleep-wake rhythm were achieved when intake occurred approximately five hours before dim light melatonin onset [120;121]. The average child with chronic sleep onset insomnia, aged 6-12 years, showed a dim light melatonin onset of approximately 20:45 h (Chapter seven), which implicates that the ideal timing of melatonin administration to synchronize sleep-wake rhythm would be at atound 15:45 h. However, pharmacological doses of melatonin result in sedation approximately one to three hours after intake [205b], which is commonly undesired when occurring in the afternoon. Although melatonin may induce less robust phase advances when taken in shortly before the desired bedtime, it will then induce its beneficial hypnotic effect at the most favorable moment. Thus, finding the ideal trade-off between the chronobiotic and sedative effects of melatonin is a quite complex, however highly important, aspect of its application in children.

In contrast to our initial hypothesis, we did not find an effect of melatonin treatment on quality of life, behavior, or cognition in medication-free children with ADHD and chronic sleep onset insomnia. Even positive responders with respect to sleep who showed advances of sleep onset of more than 30 minutes did not demonstrate improvements in behavioral or cognitive measures. That contradicts previous findings that melatonin treatment improved quality of life in children with chronic sleep onset insomnia [191]. Furthermore, it is not in line with recent findings that treatment of other sleep-related disorders such as obstructive sleep apnea exerted a positive effect on problem behavior and cognition in children with ADHD $[104 ; 145]$. Possible explanations for this discrepancy may be that melatonin treatment of insomnia needed a duration longer than four weeks to improve quality of life, behavior, or cognition. Perhaps, beneficial effects of melatonin on daytime function were too specific to be detected by generic measures, or were masked by large ADHD-related impairments in daytime function.

Our findings indicate that melatonin is only applicable for the treatment of chronic sleep onset insomnia, and not as an additional treatment option for ADHD impairments of behavior, cognition, and quality of life. Therefore, melatonin treatment for insomnia requires supplementary psychostimulant treatment for ADHD-related dysfunction. One open-label study investigated the combined use 
of melatonin and stimulants, which showed robust beneficial effects of melatonin on sleep [203]. However, further systematic studies are needed to investigate the efficacy and safety of a combined treatment of melatonin with psychostimulants.

Despite growing evidence that melatonin treatment is effective and safe in children, in many countries (e.g. the Netherlands) it is still not registered as a medicine, and is often not covered by health insurance companies. The application of melatonin treatment in children remains a topic of considerable debate since long-term effects of melatonin use in children have not sufficiently been investigated [17]. To our best knowledge, we were the first to conduct a systematic long-term follow-up of melatonin use in children (Chapter nine), in which no significant adverse events were reported. However, more systematic studies are needed that investigate potential long term adverse effects of melatonin, particularly on the gonadotropic system and puberty [77;188], and on potential proconvulsant properties [186]. Furthermore, future research should address the efficacy of alternative non-pharmacological treatment approaches such as brightlight therapy, chronotherapy, or cognitive-behavioral treatment that have been proved effective in adults [47], and children without ADHD with chronic sleep onset insomnia $[111 ; 136]$.

The question remains what the relevance of melatonin treatment is if it does not improve behavior, cognitive performance, and quality of life in children with ADHD and chronic sleep onset insomnia. Our findings showed that melatonin treatment alleviated the child's core problems, which indicates that it ameliorated the burden on the child. Thus, the application of melatonin treatment in children with ADHD and chronic sleep onset insomnia is only justified when there are at least signs that the complaints of insomnia impose a permanent negative impact on the child's life. It needs no explanation that this requires a careful and thorough history taking by the clinician. 
References 
1. A 14-month randomized clinical trial of treatment strategies for attention-deficit/hyperactivity disorder. The MITA Cooperative Group. Multimodal Treatment Study of Children with ADHD. Arch. Gen. Psychiatry, 1999, 56: 1073-1086.

2. Clinical practice guideline: diagnosis and evaluation of the child with attention-deficit/hyperactivity disorder. American Academy of Pediatrics. Pediatrics, 2000, 105: 1158-1170.

3. Clinical practice guideline: diagnosis and management of childhood obstructive sleep apnea syndrome. Pediatrics, 2002, 109: 704-712.

4. 2003 National Sleep Disorders Research Plan. Slecp, 2003, 26: 253-257.

5. Achenbach, T. M. and Achenbach, T. M. Manual for the Teacher's report form and 1991 profile. Dept. of Psychiatry, University of Vermont, Burlington, Vt, 1991.

6. Achenbach, T. M. and University of Vermont Department of Psychiatry Manual for the cbild bebanior checklist/4-18 and 1991 profile. Burlington, Vt. : Dept. of Psychiatry, University of Vermont, 1991.

7. Ahmann, P. A., Waltonen, S. J., Olson, K. A., Theye, F. W., Van Erem, A. J. and LalPant, R. J. Placebo-controlled evaluation of Ritalin side effects. Pediatrics, 1993, 91: 1101-1106.

8. Ali, N. J., Pitson, D. and Stradling, J. R. Sleep disordered breathing: effects of adenotonsillectomy on behavior and psychological functioning. Eur. J Pediatr., 1996, 155: 56-62.

9. Allen, R. P., Picchietti, D., Hening, W. A., Trenkwalder, C., Walters, A. S. and Montplaisi, J. Restless legs syndrome: diagnostic criteria, special considerations, and cpidemiology. A report from the restless legs syndrome diagnosis and epidemiology workshop at the National Institutes of Health. Sleep Med., 2003, 4: 101-119.

10. Almeida Montes, L. G., Ontiveros Uribe, M. P., Cortes, S. J. and Heinze, M. G. Treatment of primary insomnia with melatonin: a double-blind, placebo-controlled, crossover study. J. Psychiatry Neurosci., 2003, 28: 191-196.

11. American Academy of Sleep Medicine ICSD - International classification of sleep disorders, revised: Diagnostic and coding manual. Academy of Sleep Medicine, Chicago, Illinois, 2001.

12. American Psychiatric Association Diagnosic and Statistical Manual of Mental Disorders. APA, Washington, D.C, 1980 (3rd cdition).

13. Amcrican Psychiatric Association Diagnostic and Statistical Mannal of Mental Disorders. APA, Washington, D.C, 1994 (4th edition).

14. Ancoli-Isracl, S., Cole, R., Alessi, C., Chambers, M., Moorcroft, W. and Pollak, C. P. The role of actigraphy in the study of sleep and circadian rhythms. Slecp, 2003, 26: 342-392.

15. Andrade, C., Srihari, B. S., Reddy, K. P. and Chandramma, L. Melatonin in medically ill patients with insomnia: a double-blind, placebo-controlled study. J. Clin. Psychiatry, 2001, 62: 41-45.

16. Archer, S. N., Robilliard, D. L., Skene, D. J., Smits, M., Williams, A., Arendt, J. and von Schantz, M. $\Lambda$ length polymorphism in the circadian clock gene Per3 is linked to delayed slecp phase syndrome and extreme diurnal preference. Sleep, 2003, 26: 413-415.

17. Arendt, J. and Skene, D. J. Melatonin as a chronobiotic. Sleep Med. Rev, 2005, 9: 25-39. 
18. Arendt, J., Skene, D. J., Niddleton, B., Lockley, S. W. and Deacon, S. Efficacy of melatonin treatment in jet lag, shift work, and blindness. J Biol. Rhythms, 1997, 12: 604-617.

19. Bailey, J. N., Ornitz, E. M., Gehricke, J. G., Gabikian, P., Russell, A. T. and Smalley, S. L. Transmission of primary nocturnal enuresis and attention deficit hyperactivity disorder. Acta Paediatr., 1999, 88: 1364-1368.

20. Ball, J. D. and Koloian, B. Sleep patterns among ADHD children. Clin. Psychol. Rev., 1995, 15: 681691.

21. Barkley, R. A. Behavioral inhibition, sustained attention, and executive functions: constructing a unifying theory of ADHD. Psychol. Bull., 1997, 121: 65-94.

22. Barkley, R. A., McMurray, M. B., Edelbrock, C. S. and Robbins, K. Side effects of methylphenidate in children with attention deficit hyperactivity disorder: a systemic, placebo-controlled evaluation. Pediatrics, 1990, 86: 184-192.

23. Bastien, C. H., Vallieres, A. and Morin, C. M. Precipitating factors of insomnia. Behav. Sleep Med, 2004, 2: 50-62.

24. Benloucif, S., Guico, M. J., Reid, K. J., Wolfe, L. F., L'hermite-Baleriaux, M. and Zec, P. C. Stability of melatonin and temperature as circadian phase markers and their relation to sleep times in humans. J. Biol. Rhythms, 2005, 20: 178-188.

25. Berger, A. and Posner, M. I. Pathologies of brain attentional networks. Neurosci. Biobehav. Rev., 2000, 24: 3-5.

26. Biederman, J., Santangelo, S. L., Faraone, S. V., Kiely, K., Guite, J., Mick, E., Reed, E. D., Kraus, I., Jellinek, M. and Perrin, J. Clinical correlates of enuresis in ADHD and non-ADHD children. J Child Psychol. Psychiatry, 1995, 36: 865-877.

27. Blader, J. C., Koplewicz, H. S., Abikoff, H. and Foley, C. Sleep problems of elementary school children. A community survey. Arch Pediatr Adolesc Med, 1997, 151: 473-480.

28. Bojkowski, C. J., Aldhous, M. E., English, J., Franey, C., Poulton, A. L., Skene, D. J. and Arendt, J. Suppression of nocturnal plasma mclatonin and 6-sulphatoxymelatonin by bright and dim light in man. Horm. Metab Res., 1987, 19: 437-440.

29. Brouillette, R. T., Fernbach, S. K. and Hunt, C. E. Obstructive sleep apnea in infants and children. J. Pediatr., 1982, 100: 31-40.

30. Brown, F. C., Buboltz, W. C., Jr. and Soper, B. Relationship of sleep hygiene awareness, sleep hygiene practices, and slcep quality in university students. Behav. Med., 2002, 28: 33-38.

31. Brown, T. E. and Modestino, E. J. Attention-Deficit Disorders With Sleep/Arousal Disturbances. In: Brown (Ed) Attention Deficit Disorders and Comorbidities in Children, Adolescents and Adults. APP, 2000: 341-362.

32. Busch, B., Biederman, J., Cohen, L. G., Sayer, J. M., Monuteaux, M. C., Mick, E., Zallen, B. and Faraone, S. V. Correlates of $\triangle \mathrm{DHD}$ among children in pediatric and psychiatric clinics. Psychiatric Services, 2002, 53: 1103-1111. 
33. Butler, R. J. Impact of nocturnal enuresis on children and young people. Scand. J Urol. Nephrol., 2001, 35: 169-176.

34. Butler, R. J., Galsworthy, M. J., Rijsdijk, F. and Plomin, R. Genetic and gender influences on nocturnal bladder control--a study of 2900 3-year-old twin pairs. Scand. J Urol. Nephrol., 2001, 35: $177-183$.

35. Cajochen, C., Krauchi, K. and Wirz-Justice, A. Role of melatonin in the regulation of human circadian rhythms and sleep. J. Neuroendocrinol., 2003, 15: 432-437.

36. Carskadon, M. A., Wolfson, A. R., Acebo, C., Tzischinsky, O. and Seifer, R. Adolescent sleep patterns, circadian timing, and sleepiness at a transition to early school days. Sleep, 1998, 21: 871881.

37. Challamel, M.-J. and Cochat, P. Enuresis: pathophysiology and treatment. Sleep Med Rev, 1999, 3: 313-324.

38. Chang, A., Kushida, C. A., Palombini, L., Carrillo, O., Hindman, J., Hong, S., Hyde, P. and Guilleminault, C. Comparison study of actigraphic, polysomnographic, and subjective perception of sleep parameters. Sleep, 1999, 22: S43.

39. Cheek, R. E., Shaver, J. L. and Lentz, M. J. Lifestyle practices and nocturnal sleep in midlife women with and without insomnia. Biol. Res. Nurs., 2004, 6: 46-58.

40. Chervin, R. D. and Archbold, K. H. Hyperactivity and polysomnographic findings in children evaluated for sleep-disordered breathing. Sleep, 2001, 24: 313-320.

41. Chervin, R. D., Archbold, K. H., Dillon, J. E., Panahi, P., Pituch, K. J., Dahl, R. E. and Guilleminault, C. Inattention, hyperactivity, and symptoms of sleep-disordered breathing. Pediatrics, 2002, 109: 449-456.

42. Chervin, R. D., Atchbold, K. H., Dillon, J. E., Pituch, K. J., Panahi, P., Dahl, R. E. and Guilleminault, C. Associations between symptoms of inattention, hyperactivity, testless legs, and periodic leg movements. Sleep, 2002, 25: 213-218.

43. Chervin, R. D., Dillon, J. E., Bassetti, C., Ganoczy, D. A. and Pituch, K. J. Symptoms of slecp disorders, inattention, and hyperactivity in children. Slecp, 1997, 20: 1185-1192.

44. Chesson, A., Jr., Hartse, K., Anderson, W. M., Davila, D., Johnson, S., Littner, M., Wise, M. and Rafecas, J. Practice parameters for the evaluation of chronic insomnia. An American Academy of Sleep Medicine report. Standards of Practice Committec of the American Academy of Sleep Medicine. Sleep, 2000, 23: 237-241.

45. Chesson, A. L., Jr., Wise, M., Davila, D., Johnson, S., Littner, M., Anderson, W. M., Hartse, K. and Rafecas, J. Practice parameters for the treatment of restless legs syndrome and periodic limb movement disorder. An American Academy of Sleep Medicine Report. Standards of Practice Committee of the American Academy of Sleep Medicine. Sleep, 1999, 22: 961-968.

46. Cohen, J. Eta-squared and partial eta-squared in fixed factor ANOVA designs. Educational and Psychological Measurement, 1973, 33: 107-112.

47. Cole, R. J., Smith, J. S., Alcala, Y. C., Eilliott, J. A. and Kripke, D. 1:. Bright-light mask treatment of delayed sleep phase syndrome. J Biol. Rhythms, 2002, 17: 89-101. 
48. Collier, J., Butler, R. J., Redsell, S. A. and Evans, J. H. An investigation of the impact of nocturnal enuresis on children's self-concept. Scand. J Urol. Nephrol., 2002, 36: 204-208.

49. Corkum, P. Sleep Problems in Attention Deficit Hyperactivity Disorder. In: G. Stores and L. Wiggs (Eds) Sleep Disturbance in Children and Adolescents with Disorders of Development: its Significance and Management. Mac Keith Press, 2001: 174-180.

50. Corkum, P., Moldofsky, H., Hogg-Johnson, S., Humphries, T. and Tannock, R. Sleep problems in children with attention-deficit/hyperactivity disorder: impact of subtype, comorbidity, and stimulant medication. J. Am. Acad. Child Adolesc. Psychiatry, 1999, 38: 1285-1293.

51. Corkum, P., Tannock, R. and Moldofsky, H. Sleep disturbances in children with attentiondeficit/hyperactivity disorder. J Am Acad Child Adolesc Psychiatry, 1998, 37: 637-646.

52. Corkum, P., Tannock, R., Moldofsky, H., Hogg-Johnson, S. and Humphries, T. Actigraphy and parental ratings of sleep in children with attention-deficit/hyperactivity disorder (ADHD). Sleep, 2001, 24: 303-312.

53. Cornoldi, C., Marzocchi, G. M., Belotri, M., Caroli, M. G., Meo, T. and Braga, C. Working memory interference control deficit in children referred by teachers for ADHD symptoms. Neuropsychol. Dev. Cogn C. Child Neuropsychol., 2001, 7: 230-240.

54. Crabtree, V. M., Ivanenko, A and Gozal, D. Clinical and parental assessment of sleep in children with attention-deficit/hyperactivity disorder referred to a pediatric sleep medicine center. Clin. Pediatr. (Phila), 2003, 42: 807-813.

55. Crabtree, V. M., Ivanenko, A., O'Brien, L. M. and Gozal, D. Periodic limb movement disorder of sleep in children. J Sleep Res., 2003, 12: 73-81.

56. Dagan, Y., Yovel, I., Hallis, D., Eisenstein, M. and Raichik, I. Evaluating the role of melatonin in the long-term treatment of delayed sleep phase syndrome (DSPS). Chronobiol. Int., 1998, 15: 181-190.

57. Dagan, Y., Zeevi-Luria, S., Sever, Y., Hallis, D., Yovel, I., Sadeh, $\Lambda$. and Dolev, E. Sleep quality in children with attention deficit hyperactivity disorder: an actigraphic study. Psychiatry Clin. Neurosci., 1997, 51: 383-386.

58. Dahl, R. E. The impact of inadequate sleep on children's daytime cognitive function. Semin. Pediatr Neurol., 1996, 3: 44-50.

59. Dahl, R. E. and Lewin, D. S. Sleep and depression. In: G. Stores and L. Wiggs (Eds) Sleep disturbances in children and adolescents with disorders of development: its significance and management. Mac Keith Press, London, 2001.

60. Dauvilliers, Y., Morin, C., Cervena, K., Carlander, B., Touchon, J., Besset, A. and Billiard, M. Family studies in insomnia. Journal of Psychosomatic Research, 2005, 58: 271-278.

61. Dawson, D., Gibbon, S. and Singh, P. The hypothermic effect of melatonin on core body temperature: is more better? J Pineal Res., 1996, 20: 192-197.

62. Day, H. D. and Abmayr, S. B. Parent reports of sleep disturbances in stimulant-medicated children with attention-deficit hyperactivity disorder. J. Clin. Psychol., 1998, 54: 701-716. 
63. De Sonneville, L. M. J. Amsterdam Neuropsychological Tasks: a computer-aided assessment programme. In: B. P. L. M. Den Brinker, P. J. Beek, A. P. Hollander and R. T. Nieuwboer (Eds) Proceedings 6th workshop computers in de psychologie. IFBK, Amsterdam, 1996: 123-125.

64. Diagnostic Classification Steering Committee, T. M. C. ICSD - Intermational classification of sleep disorders: Diagnostic and coding manual. American Sleep Disorders Association, Rochester, Minnesota, 1990.

65. Dodge, N. N. and Wilson, G. A. Melatonin for treatment of sleep disorders in children with developmental disabilities. J Child Neurol., 2001, 16: 581-584.

66. Douglass, A. B., Bornstein, R., Nino-Murcia, G., Keenan, S., Miles, L., Zarcone, V. P., Jr., Guilleminault, C. and Dement, W. C. The Sleep Disorders Questionnaire. I: Creation and multivariate structure of SDQ. Sleep, 1994, 17: 160-167.

67. Dulcan, M. K. and Benson, R. S. AACAP Official Action. Summary of the practice parameters for the assessment and treatment of children, adolescents, and adults with ADHD. J Am Acad Child Adolesc Psychiatry, 1997, 36: 1311-1317

68. Ebisawa, T., Uchiyama, M., Kajimura, N., Mishima, K., Kamei, Y., Katoh, M., Watanabe, T., Sekimoto, M., Shibui, K., Kim, K., Kudo, Y., Ozeki, Y., Sugishita, M., Toyoshima, R., Inoue, Y., Yamada, N., Nagase, T., Ozaki, N., Ohara, O., Ishida, N., Okawa, M., Takahashi, K. and Yamauchi, T. Association of structural polymorphisms in the human period3 gene with delayed sleep phase syndrome. EMBO Rep., 2001, 2: 342-346.

69. Edinger, J. D., Bonnet, M. H., Bootzin, R. R., Doghramji, K., Dorsey, C. M., Espic, C. A., Jamieson, A. O., McCall, W. V., Morin, C. M. and Stepanski, E. J. Derivation of research diagnostic criteria for insomnia: report of an American Academy of Sleep Medicine Work Group. Sleep, 2004, 27: 15671596.

70. Eiberg, H., Shaumburg, H. L., Von Gontard, A. and Rittig, S. Linkage study of a large Danish 4generation family with urge incontinence and nocturnal enuresis. J Urol., 2001, 166: 2401-2403.

71. Epstein, R., Chillag, N. and Lavie, P. Starting times of school: effects on daytime functioning of fifth-grade children in Israel. Sleep, 1998, 21: 250-256.

72. Farber, J. M. Clinical practice guideline: diagnosis and management of childhood obstructive sleep apnea syndrome. Pediatrics, 2002, 110: 1255-1257.

73. Fava, M. Daytime sleepiness and insomnia as correlates of depression. J. Clin. Psychiatry, 2004, 65 Suppl 16: 27-32.

74. Ferguson, J. M. SSRI Antidepressant Medications: Adverse Effects and Tolerability. Prim. Care Companion. J Clin. Psychiatry, 2001, 3: 22-27.

75. Forsling, M. L., Whecler, M. J. and Williams, A. J. The effect of melatonin administration on pituitary hormone secretion in man. Clin. Endocrinol. (Oxf), 1999, 51: 637-642.

76. Garfinkel, D., Laudon, M., Nof, D. and Zisapel, N. Improvement of slecp quality in elderly people by controlled-release melatonin. Lancet, 1995, 346: 541-544.

77. Gilad, E., Laudon, M., Matzkin, H. and Zisapel, N. Evidence for a local action of mclatonin on the rat prostate. J Urol., 1998, 159: 1069-1073. 
78. Golan, N., Shahar, E., Ravid, S. and Pillar, G. Sleep disorders and daytime sleepiness in children with attention-deficit/hyperactive disorder. Sleep, 2004, 27: 261-266.

79. Goldstein, N. A., Post, J. C., Rosenfeld, R. M. and Campbell, T. F. Impact of tonsillectomy and adenoidectomy on child behavior. Arch. Otolaryngol. Head Neck Surg., 2000, 126: 494-498.

80. Gottlieb, D. J., Vezina, R. M., Chase, C., Lesko, S. M., Heeren, T. C., Weese-Mayer, D. E., Auerbach, S. H. and Corwin, M. J. Symptoms of sleep-disordered breathing in 5-year-old children are associated with sleepiness and problem behaviors. Pediatrics, 2003, 112: 870-877.

81. Gruber, R. and Sadeh, A. Sleep and neurobehavioral functioning in boys with attentiondeficit/hyperactivity disorder and no reported breathing problems. Sleep, 2004, 27: 267-273.

82. Gruber, R., Sadeh, A. and Raviv, A. Instability of sleep patterns in children with attentiondeficit/hyperactivity disorder. J. Am. Acad. Child Adolesc. Psychiatry, 2000, 39: 495-501.

83. Haimov, I., Laudon, M., Zisapel, N., Souroujon, M., Nof, D., Shlitner, A., Herer, P., Tzischinsky, O. and Lavie, P. Sleep disorders and melatonin rhythms in elderly people. BM], 1994, 309: 167.

84. Harsh, JR., Easley, A. and LeBourgeois, MK. A measure of sleep hygiene. Sleep, 2002, 25: A316.

85. Harvey, A. G. Pre-sleep cognitive activity: A comparison of sleep-onset insomniacs and good sleepers. British Journal of Clinical Psychology, 2000, 39: 275-286.

86. Harvey, A. G. Sleep hygiene and sleep-onset insomnia. J Nerv. Ment. Dis., 2000, 188: 53-55.

87. Hauri, P. J. Clinical work with insomnia. State of the art (circa 2000). In: M. P. Szuba, J. D. Kloss and D. F. Dinges (Eds) Insonnia principles and management. Cambridge University Press, Cambridge, UK, 2003: 73-82.

88. Hauri, P. J. and Wisbey, J. Wrist actigraphy in insomnia. Sleep, 1992, 15: 293-301.

89. Hayakawa, T., Kamei, Y., Urata, J., Shibui, K., Ozaki, S., Uchiyama, M. and Okawa, M. Trials of bright light exposure and melatonin administration in a patient with non-24 hour sleep-wake syndrome. Psychiatry Clin. Neurosci., 1998, 52: 261-262.

90. Hellriegel, E. T., Arora, S., Nelson, M. and Robertson, P., Jr. Steady-state pharmacokinetics and tolerability of modafinil given alone or in combination with methylphenidate in healthy volunteers. $J$ Clin. Pharmacol., 2001, 41: 895-904.

91. Herrerias, C. T., Perrin, J. M. and Stein, M. T. The child with ADHD: using the AAP Clinical Practice Guidelinc. American Academy of Pediatrics. Am Fam. Physician, 2001, 63: 1803-1810.

92. Herxheimer, A. and Petrie, K. J. Melatonin for the prevention and treatment of jet lag. Cochrane. Database. Syst. Rev, 2002, CD001520.

93. Hoban, T. F. and Chervin, R. D. Assessment of sleepiness in childten. Semin. Pediatr. Neurol., 2001, 8: $216-228$.

94. Holbrook, A. M., Crowther, R., Lotter, A., Cheng, C. and King, D. Meta-analysis of benzodiazepine use in the treatment of insomnia. CMAJ., 2000, 162: 225-233. 
95. Hryshko-Mullen, A. S. Behavioral treatment of insomnia: the Wilford Hall Insomnia Program. Mil. Med, 2000, 165: 200-207.

96. Hughes, R. J. and Badia, P. Sleep-promoting and hypothermic effects of daytime melatonin administration in humans. Sleep, 1997, 20: 124-131.

97. James, S. P., Sack, D. A., Rosenthal, N. E. and Mendelson, W. B. Melatonin administration in insomnia. Neuropsychopharmacology, 1990, 3: 19-23.

98. Jan, J. E., Espezel, H. and Appleton, R. E. The treatment of sleep disorders with melatonin. Dev. Med Child Neurol., 1994, 36: 97-107.

99. Jan, J. E., Hamilton, D., Seward, N., Fast, D. K., Freeman, R. D. and Laudon, M. Clinical trials of controlled-release melatonin in children with sleep-wake cycle disorder. J Pineal Res., 2000, 29: 34 39.

100. Jan, M. M. Melatonin for the treatment of handicapped children with severe sleep disorders. Pediatr. Neurol., 2000, 23: 229-232.

101. Jean-Louis, G., Mendlowicz, M. V., Gillin, J. C., Rapaport, M. H., Kelsoc, J. R., Zizi, F., Landolt, H. and von Gizycki, H. Sleep estimation from wrist activity in patients with major depression. Physiol Behav., 2000, 70: 49-53.

102. Jefferson, C. D., Drake, C. L., Scofield, H. M., Myers, E., McClure, T., Roehrs, T. and Roth, T. Sleep hygiene practices in a population-based sample of insomniacs. Sleep, 2005, 28: 611-615.

103. Jenni, O. G. Sleep onset insomnia during childhood or poor fit between biology and culture: comment on Van der Heijden et al. 'Prediction of melatonin efficacy by pre-treatment dim light melatonin onset in children with idiopathic chronic sleep onset insomnia'. J Sleep Res., 2005, 14: 195-197.

104. Johnstone, S. J., Tardif, H. P., Barry, R. J. and Sands, T. Nasal bilevel positive airway pressure therapy in children with a sleep-related breathing disorder and attention-deficit hyperactivity disorder: effects on electrophysiological measures of brain function. Sleep Med., 2001, 2: 407-416.

105. Kayumov, L., Brown, G., Jindal, R., Buttoo, K. and Shapiro, C. M. A randomized, double-blind, placebo-controlled crossover study of the effect of exogenous melatonin on delayed sleep phase syndrome. Psychosom. Med., 2001, 63: 40-48.

106. Kent, J. D., Blader, J. C., Koplewicz, H. S., Abikoff, H. and Foley, C. A. Effects of late-afternoon methylphenidate administration on behavior and sleep in attention-deficit hyperactivity disorder. Pediatrics, 1995, 96: 320-325.

107. Kirov, R., Kinkelbur, J., Heipke, S., Kostanecka-Endress, T., Westhoff, M., Cohrs, S., Ruther, E., Hajak, G., Banaschewski, T. and Rothenberger, $\Lambda$. Is there a specific polysomnographic sleep pattern in children with attention deficit/hyperactivity disorder? J Sleep Res., 2004, 13: 87-93.

108. Klerman, E. B., Gershengorn, H. B., Duffy, J. F. and Kronaucr, R. E. Comparisons of the variability of three markers of the human circadian paccmaker. J Biol. Rhythms, 2002, 17: 181-193.

109. Kloss, J. D. and Szuba, M. P. Insomnia in psychiatric disorders. In: M. P. Szuba, J. D. Kloss and D. F. Dinges (Eds) Insomnia principles and management. Cambridge University Press, Cambridge, UK, 2003: 43-70. 
110. Konofal, E., Lecendreux, M., Bouvard, M. P. and Mouren-Simeoni, M. C. High levels of nocturnal activity in children with attention-deficit hyperactivity disorder: a video analysis. Psychiatry Clin. Neurosci., 2001, 55: 97-103.

111. Kuhn, B. R. and Elliott, A. J. Treatment efficacy in behavioral pediatric sleep medicine. J Psychosom. Res., 2003, 54: 587-597.

112. Kuntsi, J. and Stevenson, J. Psychological mechanisms in hyperactivity: II. The role of genetic factors. J Child Psychol. Psychiatry, 2001, 42: 211-219.

113. Kushida, C. A., Chang, A., Gadkary, C., Guilleminault, C., Carrillo, O. and Dement, W. C. Comparison of actigraphic, polysomnographic, and subjective assessment of sleep parameters in sleep-disordered patients. Sleep Med., 2001, 2: 389-396.

114. Lawrence, V., Houghton, S., Douglas, G., Durkin, K., Whiting, K. and Tannock, R. Executive function and ADHD: a comparison of children's performance during neuropsychological testing and real-world activities. J. Atten. Disord., 2004, 7: 137-149.

115. LeBourgeois, M. K., Giannotti, F., Cortesi, F., Wolfson, A. and Harsh, J. Sleep hygiene and sleep quality in italian and american adolescents. Ann. N. Y. Acad Sci., 2004, 1021: 352-354.

116. LeBourgeois, MK., Giannotti, F., Cortesi, F., Wolfson, AR. and Harsh, J. The Relationship Between Reported Sleep Quality and Sleep Hygiene in Italian and American Adolescents. Pediatrics, 2005, 115: 257-265.

117. Lecendreux, M., Konofal, E., Bouvard, M., Falissard, B. and Mouren-Simeoni, M. C. Sleep and alertness in children with ADHD. J Child Psychol. Psychiatry, 2000, 41: 803-812.

118. Lee, C., Weaver, D. R. and Reppert, S. M. Direct association between mouse PERIOD and CKIepsilon is critical for a functioning circadian clock. Mol. Cell Biol., 2004, 24: 584-594.

119. Lewy, A. J. The dim light melatonin onset, melatonin assays and biological rhythm research in humans. Biol. Signals Recept., 1999, 8: 79-83.

120. Lewy, A. J., Ahmed, S., Jackson, J. M. and Sack, R. L. Melatonin shifts human circadian thythms according to a phase-response curve. Chronobiol. Int., 1992, 9: 380-392.

121. Lewy, A. J., Bauer, V. K., Ahmed, S., Thomas, K. H., Cutler, N. L., Singer, C. M., Moffit, M. T. and Sack, R. I. The human phase tesponse curve (PRC) to melatonin is about 12 hours out of phase with the PRC to light. Chronobiol. Int., 1998, 15: 71-83.

122. Lewy, A. J., Emens, J. S., Bernert, R. A. and Lefler, B. J. Eventual entrainment of the human circadian paccmaker by melatonin is independent of the circadian phase of treatment initiation: clinical implications. J Biol. Rhythms, 2004, 19: 68-75.

123. Lichstein, K. J., Durrence, H. H., Taylor, D. J., Bush, A. J. and Riedel, B. W. Quantitative criteria for insomnia. Behav. Res. Ther., 2003, 41: 427-445.

124. Littner, M., Hirshkowitz, M., Kramer, M., Kapen, S., Anderson, W. M., Bailey, D., Berry, R. B., Davila, D., Johnson, S., Kushida, C., Loube, D. I., Wise, M. and Woodson, T. Practice parameters for using polysomnography to evaluate insomnia: An update. Sleep, 2003, 26: 754-760. 
125. Littner, M., Kushida, C. A., Anderson, W. M., Bailey, D., Berry, R. B., Davila, D. G., Hirshkowitz, M., Kapen, S., Kramer, M., Loube, D., Wise, M. and Johnson, S. F. Practice parameters for the role of acrigraphy in the study of sleep and circadian rhythms: an update for 2002. Sleep, 2003, 26: 337 341.

126. Liu, X., Liu, L., Owens, J. A. and Kaplan, D. L. Sleep patterns and sleep problems among schoolchildren in the united states and china. Pediatrics, 2005, 115: 241-249.

127. Lorenzo, J. L. and Barbanoj, M. J. Variability of sleep parameters across multiple laboratory sessions in healthy young subjects: the "very first night effect". Psychophysiology, 2002, 39: 409-413.

128. Luboshitzky, R. and Lavie, P. Melatonin and sex hormone interrelationships--a review. J Pediatr Endocrinol. Metab, 1999, 12: 355-362.

129. Marcotte, A. C., Thacher, P. V., Butters, M., Bortz, J., Accbo, C. and Carskadon, M. A. Parental report of sleep problems in children with attentional and learning disorders. J Dev. Behav. Pediatr., 1998, 19: 178-186.

130. Martin, N., Scourfield, J. and McGuffin, P. Observer effects and heritability of childhood attentiondeficit hyperactivity disorder symptoms. Br. J Psychiatry, 2002, 180: 260-265.

131. McArthur, A. J. and Budden, S. S. Sleep dysfunction in Rett syndrome: a trial of exogenous melatonin treatment. Dev. Med Child Neurol., 1998, 40: 186-192.

132. McArthur, A. J., Lewy, A. J. and Sack, R. L. Non-24-hour sleep-wake syndrome in a sighted man: circadian rhythm studies and efficacy of melatonin treatment. Sleep, 1996, 19: 544-553.

133. McCall, W. V., Reboussin, B. A. and Cohen, W. Subjective measurement of insomnia and quality of life in depressed inpatients. Journal of Sleep Research, 2000, 9:43-48.

134. McGrath, A. M., Handwerk, M. L., Armstrong, K. J., Lucas, C. P. and Friman, P. C. The validity of the ADHD section of the Diagnostic Interview Schedule for Children. Bchav. Modif., 2004, 28: 349374.

135. Meijer, A. M., Habekothe, H. T. and van den Wittenboer, G. I. Time in bed, quality of sleep and school functioning of children. J Sleep Res., 2000, 9: 145-153.

136. Meltzer, L. J. and Mindell, J. A. Nonpharmacologic treatments for pediatric slecplessness. Pediatr Clin. North Am, 2004, 51: 135-151.

137. Mick, E., Biederman, J., Jetton, J. and Faraone, S. V. Slcep disturbances associated with attention deficit hyperactivity disorder: the impact of psychiatric comorbidity and pharmacotherapy. J. Child Adolesc. Psychopharmacol., 2000, 10: 223-231.

138. Middleton, B., Arendt, J. and Stone, B. M. Complex effects of melatonin on human circadian thythms in constant dim light. J. Biol. Rhythms, 1997, 12: 467-477.

139. Mikkelsen, E. J., Rapoport, J. L., Nee, L., Gruenau, C., Mendelson, W. and Gillin, J. C. Childhood enuresis. I. Sleep patterns and psychopathology. Arch. Gen. Psychiatry, 1980, 37: 1139-1144.

140. Mindell, J. A. Insomnia in childten and adolescents. In: M. P. Szuba, J. D. Kloss and D. I. Dinges (Eds) Insominia principles and management. Cambridge University Press, Cambridgc, UK, 2003: 125-135. 
141. Mullaney, D. J., Kripke, D. F. and Messin, S. Wrist-actigraphic estimation of sleep time. Sleep, 1980, 3: 83-92.

142. Nagtegaal, E., Peeters, T., Swart, W., Smits, M., Kerkhof, G. and van der Meer G. Correlation berween concentrations of melatonin in saliva and serum in patients with delayed sleep phase syndrome. Ther. Drug Monit., 1998, 20: 181-183.

143. Nagtegaal, J. E., Kerkhof, G. A., Smits, M. G., Swart, A. C. and van der Meer, Y. G. Delaycd sleep phase syndrome: A placebo-controlled cross-over study on the effects of melatonin administered five hours before the individual dim light melatonin onset. J. Sleep Res., 1998, 7: 135-143.

144. Nagtegaal, J. E., Laurant, M. W., Kerkhof, G. A., Smits, M. G., van der Meer, Y. G. and Coenen, A. M. Effects of melatonin on the quality of life in patients with delaycd slecp phase syndrome. J. Psychosom. Res., 2000, 48: 45-50.

145. Naseem, S., Chaudhary, B. and Collop, N. Attention deficit hyperactivity disorder in adults and obstructive sleep apnea. Chest, 2001, 119: 294-296.

146. National Institute of Mental Health Diagnostic Interview Scbedule for Children: Parent Informant NIMH DISC-P version 2.3). National Institute of Mental Health, Bethesda, MD, 1992.

147. Nevcus, T. The role of sleep and arousal in nocturnal enuresis. Acta Paediatr., 2003, 92: 1118-1123.

148. O'Brien, L. M., Holbrook, C. R., Mervis, C. B., Klaus, C. J., Bruner, J. I., Raffield, T. J., Rutherford, J., Mehl, R. C., Wang, M., Tuell, A., Hume, B. C. and Gozal, D. Sleep and neurobehavioral characteristics of 5- to 7-year-old children with parentally reported symptoms of attentiondeficit/hyperactivity disorder. Pediatrics, 2003, 111: 554-563.

149. O'Brien, L. M., Ivanenko, A., Crabtree, V. M., Holbrook, C. R., Bruner, J. L., Klaus, C. J. and Gozal, D. Sleep disturbances in children with attention deficit hyperactivity disorder. Pediatr. Res., 2003, 54: 237-243.

150. O'Brien, L. M., Ivanenko, A., Crabtree, V. M., Holbrook, C. R., Bruner, J. L., Klaus, C. J. and Gozal, $D$. The effect of stimulants on sleep characteristics in children with attention deficit/hyperactivity disorder. Sleep Med., 2003, 4: 309-316.

151. O'Callaghan, F. J., Clatke, A. A., Hancock, E., Hunt, A. and Osborne, J. P. Use of melatonin to treat sleep disorders in tuberous sclerosis. Dev. Med Child Neurol., 1999, 41: 123-126.

152. Olfson, M., Gameroff, M. J., Marcus, S. C. and Jensen, P. S. National trends in the treatment of attention deficit hyperactivity disorder. Am J Psychiatry, 2003, 160: 1071-1077.

153. Owens, J., Spirito, A., Marcotte, A., McGuinn, M. and Berkelhammer, L. Neuropsychological and Behavioral Correlates of Obstructive Sleep Apnea Syndrome in Children: A Prcliminary Study. Slecp Breath., 2000, 4: 67-78.

154. Owens, J. A., Maxim, R., Nobile, C., McGuinn, M. and Msall, M. Parental and self-report of sleep in children with attention-deficit/hyperactivity disorder. Arch. Pediatr. Adolesc. Mcd., 2000, 154: 549555.

155. Owens, J. A., Rosen, C. L. and Mindell, J. A. Medication use in the treatment of pediatric insomnia: results of a survey of community-based pediatricians. Pediatrics, 2003, 111: c628-c635. 
156. Owens, J. A., Spirito, A., McGuinn, M. and Nobile, C. Sleep habits and sleep disturbance in elementary school-aged children. J Dev. Behav. Pediatr, 2000, 21: 27-36.

157. Ozaki, S., Uchiyama, M., Shirakawa, S. and Okawa, M. Prolonged interval from body temperature nadir to sleep offset in patients with delayed sleep phase syndrome. Sleep, 1996, 19: 36-40.

158. Pearl, P. L. Sleep problems, stimulants, and ADHD: true, true, unrelated? Sleep Med., 2003, 4: 271 272.

159. Peled, N., Shorer, Z., Peled, E. and Pillar, G. Melatonin effect on seizures in children with severe neurologic deficit disorders. Epilepsia, 2001, 42: 1208-1210.

160. Pereira, D. S., Tufik, S., Louzada, F. M., Benedito-Silva, A. A., Lopez, A. R., Lemos, N. A., Korczak, A. L., D'Ameida, V. and Pedrazzoli, M. Association of the length polymorphism in the human Per3 gene with the delayed sleep-phase syndrome: does latitude have an influence upon it? Sleep, 2005, 28: 29-32.

161. Perlis, M. L., Giles, D. E., Buysse, D. J., Tu, X. and Kupfer, D. J. Self-reported sleep disturbance as a prodromal symptom in recurrent depression. Journal of Affective Disorders, 1997, 42: 209-212.

162. Perlis, M. L., McCall, W. V., Jungquist, C. R., Pigcon, W. R. and Matteson, S. E. Placebo effects in primary insomnia. Sleep Med. Rev., 2005, 9: 381-389.

163. Picchietti, D. L., England, S. J., Walters, A. S., Willis, K. and Verrico, T. Periodic limb movement disorder and restless legs syndrome in children with attention-deficit hyperactivity disorder. J Child Neurol., 1998, 13: 588-594.

164. Picchietti, D. L., Underwood, D. J., Farris, W. A., Walters, A. S., Shah, M. M., Dahl, R. E., Trubnick, L. J., Bertocci, M. A., Wagner, M. and Hening, W. A. Further studies on periodic limb movement disorder and restless legs syndrome in children with attention-deficit hyperactivity disorder. Mov Disord., 1999, 14: 1000-1007.

165. Rajaratnam, S. M., Dijk, D. J., Middleton, B., Stone, B. M. and Arendt, J. Melatonin phase-shifts human circadian thythms with no evidence of changes in the duration of endogenous melatonin secretion or the 24-hour production of reproductive hormones. J. Clin. Endocrinol. Metab, 2003, 88: 4303-4309.

166. Ramos Platon, M. J., Vela, B. A., Espinar, S. J. and Kales, S. Hypnopolygraphic alterations in Attention Deficit Disorder (ADD) children. Int. J Neurosci., 1990, 53: 87-101.

167. Redlin, U. Neural basis and biological function of masking by light in mammals: suppression of melatonin and locomotor activity. Chronobiol. Int., 2001, 18: 737-758.

168. Reiter, R. J. Melatonin and human reproduction. Ann. Med, 1998, 30: 103-108.

169. Rensing, L. and Ruoff, P. Temperature effect on entrainment, phase shifting, and amplitude of circadian clocks and its molecular bases. Chronobiol. Int., 2002, 19:807-864.

170. Rey, J. M., Bird, K. D. and Hensley, V. R. Bedwetting and psychopathology in adolescents. J Paediatr. Child Health, 1995, 31: 508-512. 
171. Riccio, C. A., Waldrop, J. J., Reynolds, C. R. and Lowe, P. Effects of stimulants on the continuous performance test (CP'T): implications for CPT use and interpretation. J Neuropsychiatry Clin Neurosci, 2001, 13: 326-335.

172. Ridderinkhof, K. R., Scheres, A., Oosterlaan, J. and Sergeant, J. A. Delta plots in the study of individual differences: new tools reveal response inhibition deficits in $\mathrm{AD} / \mathrm{HD}$ that are eliminated by methylphenidate treatment. J Abnorm Psychol, 2005, 114: 197-215.

173. Riedel, B. W., Winfield, C. F. and Lichstein, K. L. First night effect and reverse first night effect in older adults with primary insomnia: does anxiety play a role? 2001, 2: 125-133.

174. Ring, A., Stein, D., Barak, Y., Teicher, A., Hadjez, J., Elizur, A. and Weizman, A. Sleep disturbances in children with attention-deficit/hyperactivity disorder: a comparative study with healthy siblings. J Learn. Disabil., 1998, 31: 572-578.

175. Robilliard, D. L., Archer, S. N., Arendt, J., Lockley, S. W., Hack, L. M., English, J., Leger, D., Smits, M. G., Williams, A., Skene, D. J. and Von Schantz, M. The 3111 Clock gene polymorphism is not associated with sleep and circadian thythmicity in phenotypically characterized human subjects. J Sleep Res., 2002, 11: 305-312.

176. Robson, W. L., Jackson, H. P., Blackhurst, D. and Leung, A. K. Enuresis in childten with attentiondeficit hyperactivity disorder. South. Med. J, 1997, 90: 503-505.

177. Rosenthal, N. E., Joseph-Vanderpool, J. R., Levendosky, A. A., Johnston, S. H., Allen, R., Kelly, K. A., Souetre, E., Schultz, P. M. and Starz, K. E. Phase-shifting effects of bright morning light as treatment for delayed sleep phase syndrome. Sleep, 1990, 13: 354-361.

178. Saarenpaa-Heikkila, O., Laippala, P. and Koivikko, M. Subjective daytime sleepiness in schoolchildren. Fam. Pract., 2000, 17: 129-133.

179. Sack, R. L., Brandes, R. W., Kendall, A. R. and Lewy, A. J. Entrainment of free-running circadian thythms by melatonin in blind people. N. Engl. J. Med., 2000, 343: 1070-1077.

180. Sack, R. L., Hughes, R. J., Edgat, D. M. and Lewy, A. J. Sleep-promoting effects of melatonin: at what dose, in whom, under what conditions, and by what mechanisms? Sleep, 1997, 20: 908-915.

181. Sadeh, A., Gruber, R. and Raviv, $\Lambda$. The effects of sleep restriction and extension on school-age children: what a difference an hour makes. Child Dev., 2003, 74: 444-455.

182. Sadeh, A., Hauri, P. J., Kripke, D. F. and Lavie, P. The role of actigraphy in the evaluation of sleep disorders. Sleep, 1995, 18: 288-302.

183. Sadeh, A., Lavie, P., Scher, A., Tirosh, E. and Epstein, R. Actigraphic home-monitoring sleepdisturbed and control infants and young children: a new method for pediatric assessment of sleepwake patterns. Pediatrics, 1991, 87: 494-499.

184. Sadeh, A., Sharkey, K. M. and Carskadon, M. A. Activity-based sleep-wake identification: an empirical test of methodological issues. Sleep, 1994, 17: 201-207.

185. Sharkey, K. M. and Eastman, C. I. Melatonin phase shifts human circadian thythms in a placebocontrolled simulated night-work study. Am J Physiol Regul. Integr. Comp Physiol, 2002, 282: R454R463. 
186. Sheldon, S. H. Pro-convulsant effects of oral melatonin in neurologically disabled children. Lancet, 1998, 351: 1254.

187. Shibui, K., Uchiyama, M. and Okawa, M. Melatonin rhythms in delayed sleep phase syndrome. J. Biol. Rhythms, 1999, 14: 72-76.

188. Silman, R. E., Leone, R. M., Hooper, R. J. and Preece, M. A. Melatonin, the pineal gland and human puberty. Nature, 1979, 282: 301-303.

189. Smedje, H., Broman, J. E. and Hetta, J. Associations between disturbed sleep and behavioural difficulties in 635 children aged six to eight years: a study based on parents' perceptions. Eur. Child Adolesc Psychiatry, 2001, 10:1-9.

190. Smits, M. G., Nagtegaal, E. E., van der, H. J., Coenen, A. M. and Kerkhof, G. A. Melatonin for chronic sleep onset insomnia in children: a randomized placebo-controlled trial. J. Child Neurol., 2001, 16: 86-92.

191. Smits, M. G., van Stel, H., van der Heijden K.B., Meijer, A. M., Cocnen, A. M. and Kerkhof, G. A. Melatonin improves health status and sleep in children with idiopathic sleep onset insomnia. $A$ randomized placebo-controlled study. J Am Acad Child Adolesc Psychiatry, 2003, 42: 1286-1293.

192. Spilsbury, J. C., Storfer-Isser, A., Drotar, D., Rosen, C. L., Kirchner, H. L. and Redline, S. Effects of the home environment on school-aged children's sleep. Sleep, 2005, 28: 1419-1427.

193. Stein, M. A. Unravelling slecp problems in treated and untreated children with ADHD. J. Child $\Lambda$ dolesc. Psychopharmacol., 1999, 9: 157-168.

194. Stein, M. A., Mendelsohn, J., Obermeyer, W. H., Amromin, J. and Benca, R. Sleep and behavior problems in school-aged children. Pediatrics, 2001, 107: E60.

195. Stein, M. A., Sarampote, C. S., Waldman, I. D., Robb, A. S., Conlon, C., Pearl, P. L., Black, D. O., Seymour, K. E. and Newcorn, J. H. A dose-response study of OROS methylphenidate in children with attention-deficit/hyperactivity disorder. Pediatrics, 2003, 112: e404.

196. Stepanski, E. J. and Wyatt, J. K. Use of sleep hygiene in the treatment of insomnia. Sleep Med. Rev., 2003, 7: 215-225.

197. Stiasny, K., Oettel, W. H. and Ttenkwalder, C. Clinical symptomatology and trcatment of restless legs syndrome and periodic limb movement disorder. Sleep Med. Rev., 2002, 6: 253-265.

198. Stoohs, R. A., Blum, H. C., Suh, B. Y. and Guilleminault, C. Misinterpretation of slecp-breathing disorder by periodic limb movement disorder. Sleep Breath., 2001, 5: 131-137.

199. Sweere, Y., Kerkhof, G. A., De Weerd, A. W., Kamphuisen, H. A., Kemp, B. and Schimsheimer, R. J. The validity of the Dutch Slecp Disorders Questionnaire (SDQ). J Psychosom. Res., 1998, 45: $549-555$.

200. Takahashi, J. S. Finding new clock components: past and future. J Biol. Rhythms, 2004, 19: 339-347.

201. Takano, A., Uchiyama, M., Kajimura, N., Mishima, K., Inoue, Y., Kamei, Y., Kitajima, T., Shibui, K., Katoh, M., Watanabe, T., Hashimotodani, Y., Nakajima, T., Ozeki, Y., Hori, T., Yamada, N., Toyoshima, R., Ozaki, N., Okawa, M., Nagai, K., Takahashi, K., Isojima, Y., Yamauchi, T. and Ebisawa, T. A missense variation in human casein kinase I epsilon gene that induces functional 
alteration and shows an inverse association with circadian rhythm sleep disorders. Neuropsychopharmacology, 2004, 29: 1901-1909.

202. Tirosh, E., Sadeh, A., Munvez, R. and Lavie, P. Effects of methylphenidate on sleep in children with attention-deficient hyperactivity disorder. An activity monitor study. Am J Dis. Child, 1993, 147: $1313-1315$.

203. Tjon Pian Gi, C. V., Broeren, J. P., Starreveld, J. S. and Versteegh, F. G. Melatonin for treatment of sleeping disorders in children with attention deficit/hyperactivity disorder: a preliminary open label study. Eur. J Pediatr., 2003, 102: 554-555.

204. Todd, R. D., Rasmussen, E. R., Neuman, R. J., Reich, W., Hudziak, J. J., Bucholz, K. K., Madden, P. A. and Heath, A. Familiality and heritability of subtypes of attention deficit hyperactivity disorder in a population sample of adolescent female twins. Am J Psychiatry, 2001, 158: 1891-1898.

205. Tynjala, J., Kannas, L. and Valimaa, R. How young Europeans sleep. Health Educ. Res., 1993, 8: 6980.

205b.Tzischinsky, O. and Lavie, P. Melatonin possesses time-dependent hypnotic effects. Sleep, 1994, 17: 638-645.

206. Uchiyama, M., Okawa, M., Shibui, K., Kim, K., Tagaya, H., Kudo, Y., Kamei, Y., Hayakawa, T., Urata, J. and Takahashi, K. Altered phase relation between sleep timing and core body temperature rhythm in delayed sleep phase syndrome and non-24-hour sleep-wake syndrome in humans. Neurosci. Lett., 2000, 294: 101-104.

207. Urschitz, M. S., Guenther, A., Eggebrecht, E., Wolff, J., Urschitz-Duprat, P. M., Schlaud, M. and Poets, C. F. Snoring, intermittent hypoxia and academic performance in primary school children. Am J Respir. Crit Carc Med., 2003, 168: 464-468.

208. Valent, F., Brusaferro, S. and Barbone, F. A case-crossover study of sleep and childhood injury. Pediatrics, 2001, 107: E23.

209. Vallieres, A., Ivers, H., Bastien, C. H., Beaulieu-Bonneau, S. and Morin, C. M. Variability and predictability in sleep patterns of chronic insomniacs. J. Sleep Res., 2005, 14: 447-453.

210. Van der Heijden, K. B., Blok, M. J., Spee, K., Archer, S. N., Smits, M. G., Curfs, I. M. and W.B.Gunning No evidence to support an association of PER3 clock gene polymorphism with ADHD-related idiopathic chronic sleep onset insomnia. Biol Rhythm Res, 2005, 36: 381-388.

211. Van der Heijden, K. B., Smits, M. G. and Gunning, W. B. Sleep-related Disorders in $\Lambda D H D ; \Lambda$ review. Clin. Pediatr., 2005, 44: 201-210.

212. Van der Hcijden, K. B., Smits, M. G., Van Somcren, E. J. and Gunning, W. B. Prediction of melatonin efficacy by pretreatment dim light melatonin onset in children with idiopathic chronic sleep onset insomnia. J Sleep Res., 2005, 14: 187-194.

212b.Van der Heijden, K. B., Smits, M. G., Gunning, W. B. Sleep hygiene and actigraphically evaluated sleep characteristics in childen with Attention-Deficit/Hyperactivity Disorder and Chronic Sleep Onset Insomnia. J Slcep Res, 2005, 15: 53-60. 
213. Van der Heijden, K. B., Smits, M. G., Van Someren, E. J. W. and Gunning, W. B. Idiopathic chronic sleep onset insomnia in Attention-Deficit/Hyperactivity Disorder: a circadian rhythm sleep disorder. Chronobiol. Int., 2005, 22: 559-570.

214. Van der Heijden, K. B., Smits, M. G., Van Someren, E. J. W. and Gunning, W. B. Childhood chronic sleep onset insomnia and late sleep onset: What's the difference? J Sleep Res., 2005, 14: 197199.

215. Van der Helm-van Mil AH, Van Someren, E. J, van den, B. R., van Buchem, M. A., de Craen, A. J. and Blauw, G. J. No influence of melatonin on cerebral blood flow in humans. J Clin. Endocrinol. Metab, 2003, 88: 5989-5994.

216. Van Someren, E. J. More than a marker: interaction between the circadian regulation of temperature and sleep, age-related changes, and treatment possibilities. Chronobiol. Int., 2000, 17: 313-354.

217. Van Someren, E. J., Swaab, D. F., Colenda, C. C., Cohen, W., McCall, W. V. and Rosenquist, P. B. Bright light therapy: improved sensitivity to its effects on rest-activity thythms in Alzheimer patients by application of nonparametric methods. Chronobiol. Int., 1999, 16: 505-518.

218. Vgontzas, A. N., Bixler, E. O., Lin, H. M., Prolo, P., Mastorakos, G., Vela-Bueno, A., Kales, A. and Chrousos, G. P. Chronic insomnia is associated with nyctohemeral activation of the hypothalamicpituitary-adrenal axis: Clinical implications. Journal of Clinical Endoctinology and Metabolism, 2001, 86: 3787-3794.

219. Voderholzer, U., Al Shajlawi, A., Weske, G., Feige, B. and Riemann, D. Are there gender differences in objective and subjective sleep measures? A study of insomniacs and healthy controls. Depress. Anxiety., 2003, 17: 162-172.

220. Vogels, T., Verrips, G. H., Verloove-Vanhorick, S. P., Fekkes, M., Kamphuis, R. P., Koopman, H. M., Theunissen, N. C. and Wit, J. M. Measuring health-related quality of life in children: the development of the TACQOL, parent form. Qual. Life Res., 1998, 7: 457-465.

221. Vogels, T., Verrips, G. H. W., Koopman, H. M., Theunissen, N. C. M., Fekkes, M. and Kamphuis, R. P. TACQOL Mantual; parent form and cbild form. Leiden Child Center for Child Health and Paediatrics LUMC-TNO, Leiden, 2000.

222. Von Gontard, A., Eiberg, H., Hollmann, E., Rittig, S. and Lehmkuhl, G. Molecular genetics of nocturnal enuresis: clinical and genetic heterogeneity. Acta Pacdiatr., 1998, 87: 571-578.

223. Von Gontard, A., Mauer-Mucke, K., Pluck, J., Berner, W. and Lehmkuhl, G. Clinical behavioral problems in day- and night-wetting children. Pediatr. Nephrol., 1999, 13: 662-667.

224. Walters, A. S. Toward a better definition of the restless legs syndrome. The International Restless Legs Syndrome Study Group. Mov Disord., 1995, 10: 634-642.

225. Walters, A. S., Mandelbaum, D. E., Lewin, D. S., Kugler, S., Iingland, S. J. and Miller, M. Dopaminergic therapy in children with restless legs/periodic limb movements in sleep and ADHD. Dopaminergic Therapy Study Group. Pediatr. Neurol., 2000, 22: 182-186.

226. Weaver, D. R., Stehle, J. H., Stopa, E. G. and Reppert, S. M. Melatonin receptors in human hypothalamus and pituitary: implications for circadian and reproductive responses to melatonin. J Clin. Endocrinol. Metab, 1993, 76: 295-301. 
227. Webster, J. B., Kripke, D. F., Messin, S., Mullaney, D. J. and Wyborney, G. An activity-based sleep monitor system for ambulatory use. Sleep, 1982, 5: 389-399.

228. Wechsler, D. Wecbsler Intelligence Scale for Children. Harcourt Brace Jovanovich, Toronto, 1991 (3rd edition).

229. Weinberg, W. A. and Brumback, R. A. Primary disorder of vigilance: a novel explanation of inattentiveness, daydreaming, boredom, restlessness, and sleepiness. J Pediatr., 1990, 116: 720-725.

230. Weinberg, W. A. and Harper, C. R. Vigilance and its disorders. Neurol. Clin., 1993, 11: 59-78.

231. Wiggs, L. and Stores, G. Behavioural treatment for sleep problems in children with severe learning disabilities and challenging daytime behaviour: effect on daytime behaviour. J Child Psychol. Psychiatry, 1999, 40: 627-635.

232. Wyatt, J. K. Delayed sleep phase syndrome: pathophysiology and treatment options. Sleep, 2004, 27: 1195-1203.

233. Yang, C. M., Spielman, A. J., D'Ambrosio, P., Serizawa, S., Nunes, J. and Birnbaum, J. A single dose of melatonin prevents the phase delay associated with a delaycd weekend slecp pattern. Sleep, 2001, 24: $272-281$.

234. Yilmaz, B., Kutlu, S., Mogulkoc, R., Canpolat, S., Sandal, S., Tarakci, B. and Kelestimur, H. Melatonin inhibits testosterone secretion by acting at hypothalamo-pituitary-gonadal axis in the rat. Neuroendocrinol. Lett., 2000, 21: 301-306.

235. Zaidan, R., Geoffriau, M., Brun, J., Taillard, J., Bureau, C., Chazot, G. and Claustrat, B. Melatonin is able to influence its secretion in humans: description of a phase-response curve. Neuroendocrinology, 1994, 60: 105-112.

236. Zeitzer, J. M., Dijk, D. J., Kronauer, R, Brown, E. and Czeisler, C. Sensitivity of the human circadian pacemaker to nocturnal light: melatonin phase resetting and suppression. J Physiol, 2000, 526 Pt 3: 695-702.

237. Zhdanova, I. V., Wurtman, R. J., Lynch, H. J., Ives, J. R., Dollins, A. B., Morabito, C., Matheson, J. K. and Schomer, D. L. Sleep-inducing effects of low doses of melatonin ingested in the evening. Clin. Pharmacol. Ther., 1995, 57: 552-558.

238. Zhdanova, I. V., Wurtman, R. J., Regan, M. M., Taylor, J. A., Shi, J. P. and Leclair, O. U. Mclatonin treatment for age-related insomnia. J. Clin. Endocrinol. Metab, 2001, 86: 4727-4730. 

Appendices 


\section{The Sleep Questionnaire for Parents of Children with ADHD}

\section{K.B. van der Heijden, W.B. Gunning, M.G. Smits}

Name:

Gender:

Date of birth:

Date questionnaire completed:

E-mail address:

At what age was your child when the diagnosis of ADHD was made?

Which of the following diagnoses have been made by a physician or psychologist? Depressive disorder, anxiety disorder, tic disorder/Gilles de la Tourette, motor disorder, mental disability, disruptive behavior disorder, autistic disorder/pervasive developmental disorder, speech/language disorder.

What age was your child at that time?

At what time does your child usually go to bed?

How long does it usually take your child to fall asleep?

At what time does your child usually wake up?

How does your child wake up in the morning? (spontaneously at $\ldots h /$ is woken up and leaves bed easily / is woken up and has difficulties leaving bed)

The following questions can be answered by "never", "occasionally", "often"

Does your child show resistance to go to bed?

Is your child afraid of sleeping in the dark?

Does your child leave his/her bedroom in the evening?

Does he/she have problems with bed-wetting?

Have you heard your child talking during his/her sleep?

Does your child grind his/her teeth during sleep?

Have you heard your child snoring during his/her sleep?

Does he/she wake up at night?

Does your child have nightmares?

Does your child get up to go to the parent's bedroom during the night?

Is he/she a restless sleeper?

Have you observed repetitive actions such as rocking or head banging in bed?

Does your child have difficulties waking up in the morning?

Does your child wake up too early in the morning? 
Does your child wake up in a bad mood?

Does your child fall asleep during the daytime?

Did your child ever use sleep medication? (Yes/No). If Yes: What medication? / Which dose? / At what age? / What for? / What was the effect?

Does your child use ADHD medication? (Yes/No). If Yes: What medication? Following the start with ADHD medication: a) Sleep onset problems started; b) Existing sleep onset problems got worse; c) Sleep remained the same; d) Existing sleep onset problems improved.

Are there family members who have or had ADHD? (Yes/No). If Yes: Who?

Are there family members who have had sleep onset problems during childhood?

(Yes/No). If Yes: Who? 


\section{$\mathrm{S}_{\text {laap }} \mathrm{H}_{\text {ygiene }} \mathrm{B}_{\text {alans voor }} \mathrm{K}_{\text {inderen }}$}

The University of Southern Mississippi - Laboratorium voor Slaaponderzoek formulier voor ouderrapportage over kinderen

\section{Vertaling K.B. van der Heijden, W.B. Gunning, M.G. Smits}

\section{Aanwijzingen}

Omcirkel een van de onderstaande mogelijkheden om aan te geven hoe vaak de volgende dingen gedurende de afgelopen maand zijn gebeurd.

\section{Nooit}

Zelden

Soms

Vaak

Vrijwel altijd

Altijd

\begin{tabular}{|c|c|c|c|c|c|c|c|}
\hline & & & & & & & tijd \\
\hline & & & & Vrij & vel a & ltijd & \\
\hline & & & & & aak & & \\
\hline & & & & ms & & & \\
\hline & & & den & & & & \\
\hline & & Tooit & & & & & \\
\hline & de 4 uur voor het naar bed gaan ... & & & & & & \\
\hline 1. & ... doet mijn kind een dutje. & $\mathrm{N}$ & Z & $\mathrm{S}$ & $\mathrm{V}$ & $\mathrm{VA}$ & A \\
\hline 2. & $\begin{array}{l}\text {...drinkt mijn kind iets met cafeine erin (b.v.: cola, } \\
\text { chocola, (ijs)thee, koffie). }\end{array}$ & $\mathrm{N}$ & Z & $\mathrm{S}$ & V & $\mathrm{VA}$ & A \\
\hline & t voordat mijn kind naar bed gaat ... & & & & & & \\
\hline 3. & $\begin{array}{l}\text {... doet het ontspannende dingen (b.v.: luisteren naar een } \\
\text { verhaal of muziek, lezen). }\end{array}$ & $\mathrm{N}$ & Z & $\mathrm{S}$ & $\mathrm{V}$ & VA & A \\
\hline 4. & ...drinkt het veel vloeistof. & $\mathrm{N}$ & $z$ & S & $\mathrm{V}$ & VA & A \\
\hline
\end{tabular}




\begin{tabular}{|c|c|c|c|c|c|c|c|}
\hline 5. & $\begin{array}{l}\text {... speelt het wilde spelletjes (b.v.: rennen, springen, } \\
\text { stoeien). }\end{array}$ & $\mathrm{N}$ & $\mathrm{Z}$ & $S$ & $\mathrm{~V}$ & VA & A \\
\hline 6. & $\begin{array}{l}\text {...doet dingen die hem/haar waakzaam en actief maken } \\
\text { (b.v.: videospelletjes spelen, tv kijken). }\end{array}$ & $\mathrm{N}$ & $\mathrm{Z}$ & $S$ & V & $\mathrm{VA}$ & $A$ \\
\hline \multicolumn{2}{|r|}{ Mijn kind gaat naar bed... } & & & & & & \\
\hline 7. & ...elke avond op ongeveer dezelfde tijd. & $N$ & $\mathrm{Z}$ & $\mathrm{S}$ & $\mathrm{V}$ & VA & A \\
\hline 8. & ...en klaagt daarbij over honger. & $\mathrm{N}$ & Z & S & $\mathrm{V}$ & $\mathrm{VA}$ & A \\
\hline 9. & ... en doet dingen in bed die hem/haar wakker houden. & $\mathrm{N}$ & Z & S & $\mathrm{V}$ & $\mathrm{VA}$ & $A$ \\
\hline 10. & ...elke avond op dezelfde plek. & $\mathrm{N}$ & $\mathrm{Z}$ & $\mathrm{S}$ & $\mathrm{V}$ & VA & A \\
\hline 11. & ...en is overstuur. & $N$ & $\mathrm{Z}$ & $\mathrm{S}$ & $\mathrm{V}$ & $\mathrm{VA}$ & A \\
\hline 12. & ...met zorgen. & $\mathrm{N}$ & $\mathrm{Z}$ & $\mathrm{S}$ & $\mathrm{V}$ & VA & A \\
\hline \multicolumn{2}{|c|}{ Mijn kind slaapt ... } & & & & & & \\
\hline 13. & ....in een verduisterde kamer. & $\mathrm{N}$ & Z & $\mathrm{S}$ & $\mathrm{V}$ & $\mathrm{VA}$ & A \\
\hline 14. & ...in een kamer waar het te warm of te koud is. & $\mathrm{N}$ & $\mathrm{Z}$ & $\mathrm{S}$ & $\mathrm{V}$ & VA & A \\
\hline 15. & ...in een kamer waar veel lawaai is. & $\mathrm{N}$ & Z & $S$ & $\mathrm{~V}$ & VA & A \\
\hline 16. & ...alleen (in zijn/haar eigen bed). & $\mathrm{N}$ & $\mathrm{Z}$ & $\mathrm{S}$ & $\mathrm{V}$ & $\mathrm{VA}$ & A \\
\hline 17. & ....in een niet-geluchte kamer. & $\mathrm{N}$ & $\mathrm{Z}$ & $\mathrm{S}$ & $\mathrm{V}$ & VA & A \\
\hline 18. & $\begin{array}{l}\text {...de hele nacht of een deel daarvan bij iemand anders } \\
\text { (b.v.:ouder, zus, broer). }\end{array}$ & $\mathrm{N}$ & $\mathrm{Z}$ & $S$ & V & $\mathrm{VA}$ & A \\
\hline 19. & ...in een comfortabel bed . & $N$ & $\mathrm{Z}$ & S & $\mathrm{V}$ & $\mathrm{VA}$ & $A$ \\
\hline 20. & ...in een huis waar iemand rookt (binnenshuis). & $\mathrm{N}$ & $\mathrm{Z}$ & $\mathrm{S}$ & $\mathrm{V}$ & $\mathrm{VA}$ & A \\
\hline \multicolumn{2}{|c|}{ Mijn kind... } & & & & & & \\
\hline 21. & $\begin{array}{l}\text {...heeft een tot rust brengende routine voor het slapen } \\
\text { gaan. }\end{array}$ & $\mathrm{N}$ & $Z$ & $\mathrm{~S}$ & $\mathrm{~V}$ & $\mathrm{VA}$ & A \\
\hline 22. & $\begin{array}{l}\text {...gebruikt zijn/haar bed voor andere dingen dan slapen } \\
\text { (b.v.: spelen, to kijken, videospelletjes spelen, tijd } \\
\text { doorbrengen als het voor straf naar zijn/haar kamer is } \\
\text { gestuurd). }\end{array}$ & $\mathrm{N}$ & $Z$ & $\mathrm{~S}$ & V & $\mathrm{VA}$ & $A$ \\
\hline 23. & ...wordt in bed gelegd nadat hij/zij al in slaap is gevallen. & $\mathrm{N}$ & Z & $\mathrm{S}$ & $\mathrm{V}$ & $\mathrm{VA}$ & A \\
\hline
\end{tabular}




\section{Slaap Hygiëne Balans voor Kinderen (vervolg)}

\begin{tabular}{|c|l|c|c|c|c|c|c|}
\hline 24. & .. blijft later op dan zijn/haar gebruikelijke bedtijd. & $\mathrm{N}$ & $\mathrm{Z}$ & $\mathrm{S}$ & $\mathrm{V}$ & $\mathrm{VA}$ & $\mathrm{A}$ \\
\hline 25. & $\begin{array}{l}\text {...komt 's morgens telkens op ongeveer dezelfde tijd uit } \\
\text { bed. }\end{array}$ & $\mathrm{N}$ & $\mathrm{Z}$ & $\mathrm{S}$ & $\mathrm{V}$ & VA & A \\
\hline
\end{tabular}




\section{Definition of sleep outcome parameters}

\section{Actigraphy}

- Sleep onset_act: the start of a period of at least 10 minutes of consecutively recorded immobile actigraphy data following lights out.

- Sleep latency_act: the time from lights out until sleep onset.

- Wake up time -acr: $_{\text {: }}$ the last epoch of actigraphically assessed immobility before the start of a 10 minute consecutive period of activity around the time of leaving bed.

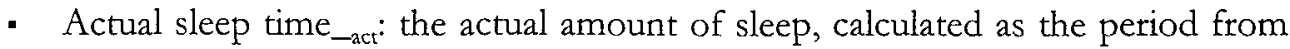
sleep onset to wake up time minus estimated time awake in the period from sleep onset until awake time.

- Sleep efficiency act: $_{\text {: }}$ the percentage of time spent asleep in the period from lights out until the time of leaving bed.

- Movingtime ${ }_{-a c t}$ : the percentage of time spent moving during the assumed sleep period.

- IS -act: Interdaily stability; the degree of resemblance between the activity patterns on individual days (range from 0 to 1 , higher values indicating more stable rhythms).

- IV $V_{-a c t}$ Intradaily variability; the fragmentation of periods of rest (or sleep) and activity (or wakefulness) (tange from 0 to 2, with higher values indicating more fragmented rhythms).

- L5_ac: the average activity during the least active five-hours period in the average 24-hour activity rhythm.

\section{Sleep $\log$}

- Lights out_log: clock time when the child lies in bed, lights are dimmed and the child is expected to go to sleep, as reported by the parents.

- Difficulty falling asleep_log: averaged score (over 7 days) on an item asking parents how difficult it was for the child to fall asleep in the evening $(1=$ not difficult; $5=$ very difficult). 


\section{Calculation of the required sample size}

A power analysis conducted prior to commencement of the study yielded an estimated required sample size of 54 children per arm (total=108), assuming an advance of mean ( $\pm \mathrm{SD}$ ) sleep onset of 1:00 $\pm 0: 50 \mathrm{~h}$ in the melatonin group and $0: 25 \pm 0: 50 \mathrm{~h}$ in placebo, a total drop-out and missing value percentage of $10 \%$ (based on previous studies,[190;191] a power (i.e. the probability that the test will reject the hypothesis tested when a specific alternative hypothesis is true) of 0.90 , and an alpha (i.e. the probability of rejecting the statistical hypothesis tested when in fact, that hypothesis is true ) of 0.05 . 
Samenvatting 
De aandachtstekorthyperactiviteitsstoomis (ADHD) is met een prevalentie van 3 tot $5 \%$ een van de meest voorkomende psychiatrische stoornissen van de kindertijd. ADHD kenmerkt zich door symptomen van inattentie, hyperactiviteit/ impulsiviteit, of beiden en resulteert in een disfunctioneren dat verstrekkende en langdurige gevolgen kan hebben. Vaak gaat ADHD gepaard met slaapstoornissen. De chronische inslaapstoornis, gekenmerkt door een persistent onvermogen om 's avonds op het gewenste tijdstip in slaap te vallen, wordt het meest frequent gerapporteerd. De co-morbide presentatie van ADHD en slaapstoornissen is in klinisch opzicht van groot belang omdat slaapstoornissen een negatieve invloed hebben op gedrag, cognitie en stemming en daarmee de ernst van de ADHD symptomen kunnen versterken. Over de etiologie (ziekteoorzaak) en behandeling van chronische inslaapstoornissen bij kinderen met $A D H D$ is nog veel onbekend.

In het kader van dit proefschrift is een aantal grootschalige onderzoeken verricht naar mogelijke oorzakelijke factoren van chronische inslaapstoornissen bij kinderen met $\mathrm{ADHD}$, alsmede naar de toepassing van melatonine voor de behandeling van chronische inslaapstoornissen bij deze kinderen. In het eerste hoofdstuk wordt ingegaan op de achtergrond en hypotheses van de verschillende onderzoeken waaruit dit proefschrift bestaat.

Et werd een literatuuronderzoek uitgevoerd naar de resultaten van dertig onderzoeken naar slaapstoornissen bij ADHD die zijn gepubliceerd sinds de introductie van het huidige psychiatrische classificatiesysteem (DSM-IV) (Hoofdstuk twee). De resultaten van het onderzoek tonen aan dat er gefundeerd wetenschappelijk bewijs is voor een relatie tussen ADHD en slaapgerelateerde stoornissen zoals de chronische inslaapstoornis, enuresis nocturna, overmatige nachtelijke beweeglijkheid, slaaparchitectuurstoornissen en overmatige slaperigheid overdag.

De belangrijkste risicofactoren van chronische inslaapstoornissen in een klinische populatie kinderen met ADHD werden door middel van een retrospectief, crosssectioneel vragenlijstonderzoek onderzocht (Hoofdstuk drie). De resultaten lieten zien dat na een start met psychostimulantia, de eerste-keus medicatie bij ADHD, er bij circa $20 \%$ van de kinderen een chronische inslaapstoornis ontstond en bij circa $15 \%$ een reeds bestaande chronische inslaapstoornis verergerde. De prevalentie van chronische inslaapstoornissen was $45.5 \%$ bij de kinderen met psychostimulantia en $28.6 \%$ bij de medicatievrije kinderen. Beide percentages liggen aanzienlijk hoger dan de geschatte prevalentie in de algemene kinderpopulatie (8-15\%). Naast het gebruik van psychostimulantia waren andere belangrijke risicofactoren: aanwezigheid van een co-morbide depressieve stoornis en weerstand bij het naar bed gaan. Chronische inslaapstoornissen in de kindertijd kwamen vaker voor bij 
eerstegraads familieleden van kinderen met $\mathrm{ADHD}$ en een chronische inslaapstoornis dan bij controles met ADHD zonder inslaapstoornis, wat wijst op een mogelijke predisponerende rol van genetische factoren.

Slaaphygiëne staat voor alle gedrags- en omgevingsfactoren rondom het naar bed gaan die de slaap kunnen beïnvloeden. We hebben de rol van slaaphygiëne in de etiologie van chronische inslaapstoornissen onderzocht bij medicatievrije kinderen met ADHD (Hoofdstuk vier). Met behulp van de gevalideerde Slaaphygiëne Balans voor Kinderen maten we het niveau van slaaphygiëne bij 74 medicatievrije kinderen met gediagnosticeerde ADHD en chronische inslaapstoornis en bij 23 controles met ADHD zonder inslaapstoornis. Tevens werd het slaap-waak ritme gemeten met behulp van actigrafie (een bewegingsmeter om de pols). De resultaten lieten geen groepsverschillen zien in het niveau van slaaphygiëne, ondanks een significant latere inslaaptijd en langere slaaplatentie bij de kinderen met ADHD en een chronische inslaapstoornis. Voorts kwamen de gemeten niveaus van slaaphygiëne overeen met wat in een eerder onderzoek was gevonden in de algemene kinderpopulatie. Deze resultaten wijzen erop dat slaaphygiëne geen belangrijke etiologische factor rol speelt in de pathogenese van chronische inslaapstoornissen bij medicatievrije kinderen met ADHD.

Bevindingen uit een voorstudie gaven aanwijzingen dat medicatievrije kinderen met ADHD en een chronische inslaapstoornis een vertraagd slaap-waak ritme en een vertraagde stijging van de concentratie van melatonine in de avond vertoonden. Melatonine is een hormoon dat 's avonds bij het donker worden wordt geproduceerd in de epifyse van de hersenen. Dit gebeurt op instigatie van de suprachiasmatische kern in de hersenen, ook wel de biologische klok genoemd, dat een endogeen 24-uurs (circadiaan) ritme heeft. Het tijdstip waarop de melatonine productie op gang komt noemt men 'dim light melatonin onset' en wordt beschouwd als de beste indicator van het endogene ritme van de biologische klok. Hoofdstuk vijf beschrijft een onderzoek waarin 'dim light melatonin onset' (gemeten uit speeksel) en het slaap-waak ritme (gemeten met actigrafie) werd gemeten bij 87 medicatievrije kinderen met een gediagnosticeerde ADHD en chronische inslaapstoornis en bij 33 controles met ADHD zonder inslaapstoornis. $\mathrm{Bij}$ de kinderen met $\mathrm{ADHD}$ en een chronische inslaapstoornis vonden we ten opzichte van ADHD controles zonder inslaapstoornis een vertraagde 'dim light melatonin onset' en een vertraagd slaap-waak ritme zonder afwijkingen in de continuiteit van de slaap. Deze bevindingen toonden voor het eerst aan dat de chronische inslaapstoornis bij medicatievrije kinderen met ADHD beschouwd moet worden als een circadiane-ritme slaapstoornis, waarbij het ritme van de biologisch klok later staat afgesteld dan gewenst. 
In recent onderzoek bij volwassenen met een circadiane-titme slaapstoornis werd een associatie gevonden met een variable number tandem repeat (VNTR) polymorfisme van het $P E R 3$ gen van de biologische klok. Hoofdstuk zes beschrijft een onderzoek dat wij verrichtten naar dit PER3 polymorfisme bij een kleine groep kinderen met ADHD en een chronische inslaapstoornis die een zeer sterk vertraagde 'dim light melatonin onset' lieten zien, alsmede bij een groep van normale controles. Er werd geen associatie gevonden tussen het PER3 polymorfisme en de chronische inslaapstoornis bij ADHD, wat erop wijst dat andere klokgenen dan wel andere intrinsieke of extrinsieke factoren betrokken zijn bij de pathogenese van chronische inslaapstoornissen bij kinderen met ADHD.

Melatonine heft, wanneer het op het juiste tijdstip wordt toegediend, een chronobiotische werking waarbij het biologische ritmes van het menselijk lichaam verschuift. Onderzoek heeft aangetoond dat melatonine een effectief middel is bij circadiane-ritme slaapstoornissen. Het bevindt zich echter nog in de onderzoeksfase en is in ons land niet geregistreerd als geneesmiddel.

Zowel de richting als grootte van de verschuiving van de biologische ritmes door melatonine zijn afhankelijk van het tijdstip van toediening binnen het endogene circadiane ritme. We onderzochten of het gebruik van melatonine in de vroege avond (tussen zes en zeven uur) effectiever was bij kinderen met chronische inslaapstoornissen met een vertraagde waarde van 'dim light melatonin onset' (Hoofdstuk zeven en acht). Er werd een grote meta-analyse uitgevoerd bij 110 kinderen met chronische inslaapstoornissen die hadden meegedaan aan twee eerder uitgevoerde gerandomiseerde, placebogecontroleerde, dubbelblinde trials naar de effectiviteit van melatonine. Voor het eerst werd aangetoond dat melatonine toegediend in de vroege avond effectiever is bij kinderen met een relatief vertraagde 'dim light melatonin onset'.

De effectiviteit van melatonine bij medicatievrije kinderen met ADHD en een chronische inslaapstoornis was niet eerder onderzocht. Onderzoek hiernaar was echter om een tweetal redenen van speciaal belang. Ten eerste vonden we in een eerder onderzoek bij deze groep een vertraagde 'dim light melatonin onset' (Hoofdstuk vijf). Een ander onderzoek had uitgewezen dat zo'n vertraagde 'dim light melatonin onset' een sterker normaliserend effect van melatonine op het slaap-waak ritme voorspelde (Hoofdstuk zeven). Ten tweede had eerder onderzoek aangetoond dat een behandeling van chronische slaap-gerelateerde stoornissen (waaronder geen inslaapstoornissen) een verbetering van ADHD-symptomen tot gevolg had. Wij verwachtten daarom van een behandeling van chronische inslaapstoornissen een zelfde effect op ADHD-symptomen, wat belangrijke 
gevolgen zou kunnen hebben op de behandelings-strategieën van ADHD in de gezondheidszorg.

In een grote gerandomiseerde, placebogecontroleerde, dubbelblinde trial onderzochten we daarom het effect van een vier weken durende melatonine behandeling op het slaap-waak ritme (gemeten met actigrafie en logboek), 'dim light melatonin onset' (gemeten uit speeksel), gedragsproblemen (vragenlijsten door ouders en leerkracht), cognitieve prestaties (neuropsychologische tests) en kwaliteit van leven (vragenlijsten). De resultaten lieten zien dat in de melatoninegroep $(n=53)$ ten opzichte van de placebogroep $(n=52)$ de subjectieve en objectieve metingen van slaap significant verbeterde en 'dim light melatonin onset' naar een vroeger tijdstip verschoof. Ouders gaven aan dat melatonine de individuele kernproblemen van het kind (snel boos worden [36.0\%], inslaapproblemen [31.8\%], aandachtsproblemen [23.3\%]) verbeterde. Er werd echter geen significante verbetering gevonden van probleemgedrag, cognitieve prestaties of kwaliteit van leven ten opzichte van placebo. $\mathrm{Na}$ vier weken noch na twee jaar werden significante bijwerkingen gevonden.

De resultaten van dit onderzoek toonden ondubbelzinnig aan dat melatonine klinisch toegepast kan worden bij medicatievrije kinderen met ADHD voor de behandeling van chronische inslaapstoornissen, maar niet voor ADHD-symptomen en uitsluitend indien er een indicatie is dat het slaapprobleem een significant lijden veroorzaakt bij het kind.

Gezamenlijk hebben de verschillende onderzoeken zoals beschreven in dit proefschrift geleid tot een beter inzicht in de etiologie en behandeling van chronische inslaapstoornissen bij kinderen met ADHD. De chronische inslaapstoornis bij kinderen met $\mathrm{ADHD}$ is een circadiane-ritme slaapstoornis waarbij het ritme van de biologisch klok later staat afgesteld dan gewenst is. Derhalve is melatoninebehandeling de aangewezen behandelingsstrategie want het normaliseert het ritme van de biologische klok en verbetert daarmee de inslaapstoornis. Ons onderzoek vond geen negatieve korte of lange termijn effecten van melatonine bij kinderen met ADHD. Echter meer onderzoek hiernaar is nodig. Vooralsnog lijkt de toepassing van melatonine bij kinderen met ADHD gerechtvaardigd indien: 1) er sprake is van chronische en ernstige inslaapproblemen die een significant lijden veroorzaken bij het kind; 2) na een verbetering van mogelijke achterliggende extrinsieke oorzaken; 3) bij voorkeur wanneer er sprake is van een vertraagde 'dim light melatonin onset'. 

Dankwoord 
De voltooiing van dit proefschrift betekent het einde van mijn bestaan als promovendus. Aan de top is er thans het uitzicht. Daarbij komt een dubbelzinnig gevoel naar boven. Enerzijds weemoed naar een fantastisch vrije, leerzame en interessante periode. Anderzijds blijdschap dat er een einde is gekomen aan het ploeteren via omwegen, onverharde -, doodlopende - en niet-bestaande wegen. Er is een groot aantal mensen dat mij heeft bijgestaan en geholpen op de weg hier naar toe. Ik ben hen allen ontzettend veel dank verschuldigd!

Allereerst en bovenal gaat mijn dank uit naar mijn promotoren en copromotor. Boudewijn, het waren prettige jaren van intensieve samenwerking en veel veranderingen. Kort na mijn start vertrok jij als hoogleraar uit het Academisch Medisch Centrum Amsterdam naar Kempenhaeghe in Heeze en ik koos er voor jou te volgen. Het bleek een wijs besluit want je was een geweldige promotor met onvoorwaardelijke steun en vertrouwen en een enorme inzet. We hebben samen bergen verzet met het diagnostisch onderzoek van meer dan 250 kinderen in het kader van dit promotieonderzoek. Ook daarbij heb ik ontzettend veel van jou geleerd. Bedankt!

Marcel, mijn copromotor, ik herinner me goed ons eerste overleg in 2001. Een nieuwe onderzoeksbevinding dat ik ter sprake bracht werd door jou direct afgedaan als "niet relevant voor je promotieonderzoek". En je had gelijk. Gelukkig heb je me daarna enorm veel kunnen bijbrengen over prioriteiten stellen, doelgericht, efficiënt en publicatiegericht werken, maar ook hoe je creatief kunt zijn in het doen van wetenschappelijk onderzoek.

Hans en Bert, jullie hebben je als promotoren in de laatste fase van mijn promotieonderzoek ingezet om mij bij Kempenhaeghe en het Academisch Ziekenhuis Maastricht de extra tijd, faciliteiten en steun te bieden die ik hard nodig had om het promotieonderzoek naar behoren te kunnen afronden.

Ik wil alle leden van de beoordelingscommissie graag bedanken voor de tijd en energie voor de beoordeling van dit proefschrift. Eus van Someren, zonder jouw ingenieuze computerprogrammaatjes was ik nu nog bezig met actigrafie files scoren...Ik heb daarnaast erg veel gehad aan je expertise en redigeerwerk! Richard Ridderinkhof, jou ben ik zeer dankbaar voor het monnikenwerk dat je hebt verricht voor de data-analyse van de flankertaak. Gerard Kerkhof en Ruud Buijs wil bedanken voor de behulpzame adviezen in de opzet- en aanvangsfase van het onderzoek. Inge van Geijlswijk, bijzonder bedankt voor het randomiseren, stratificeren en klaarmaken van de onderzoeksmedicatie. Een ingewikkelde en belangrijke klus!

Jan Gerver en Laurens Vlasveld, bedankt voor de hulp die jullie hebben geboden bij de organisatie en uitvoer van het dossier- en vragenlijstonderzoek en voor de 
hulp met de verwijzing van kinderen voor deelname aan ons onderzoek. Dank ook aan Willemijn Veenboer, mijn eerste en enige stagiair, voor je hulp bij het dossieren vragenlijstonderzoek. Michel Varkevisser, nadat we elkaar na de propedeuse psychologie in Leiden uit het oog hadden verloren, ben ik je na jaren gelukkig weer tegengekomen. Bedankt voor het uitstekende redigeerwerk an dit proefschrift! Jasper Middelberg, bedankt voor alle energie die je hebt gestopt in het ontwerp van de omslag van dit proefschrift. Het is prachtig geworden!

Hans de Groen en Johan Arends wil ik bedanken voor de hulp en begeleiding bij mijn onderzoeksactiviteiten op Kempenhaeghe, dr. W.A.C. Swart van het laboratorium van Ziekenhuis Gelderse Vallei Ede voor de uitvoer van de melatonine analyses, Rien Blok, Kim Spee en Paul Curfs van Capaciteitsgroep Genetica en Celbiologie, afdeling DNA van de Universiteit Maastricht voor de hulp bij de analyses van klokgenen en de heer Van Proosdij van der Werf van Fypro, Actiwatch Cambridge Neurotechnology voor de hulp en informatie bij de actometers.

Slapeloze nachten heb ik gehad om de trage groei van deelnemers, want zonder data geen onderzoek. Hier gold: wie een kuil graaft voor zichzelf valt erin. De strenge criteria waaraan deelnemers moesten voldoen dwongen mij er toe hulp te zoeken bij meer dan twintig gezondheidszorginstellingen over het gehele land om aan het benodigde aantal proefpersonen te komen. Ik wil hierbij Richard Hees, kinder- en jeugd psychiater van GGZ Centrum Land van Cuijk te Boxmeer hartelijk bedanken. Jij hebt je vanaf het begin van het onderzoek in 2001 tot op heden intensief ingezet voor het vinden en enthousiasmeren van onderzoeksdeelnemers en het ter beschikking stellen van alle benodigde faciliteiten op de instelling. Verder heel veel dank aan alle betrokken verwijzers, stafleden en secretaresses van de volgende instellingen: Ziekenhuis Gelderse Vallei te Ede; Epilepsiecentrum Kempenhaeghe te Heeze; De Gelderse Roos Veluwe Vallei te Ede; GGZ Amersfoort; GGZ Centrum Land Van Cuijk te Boxmeer; Rode Kruis Ziekenhuis te Beverwijk; GGZ Delfland en het Reinier De Graaf Gasthuis te Delft; GGZ Midden-Brabant te Tilburg; RIAGG IJsselland te Deventer; GGZ Spatie te Apeldoorn; GGZE te Eindhoven; Jeroen Bosch Ziekenhuis te 's-Hertogenbosch; St. Jans Gasthuis te Weert; Elkerliek Ziekenhuis te Helmond; Sint Joseph Ziekenhuis te Veldhoven; St. Annaziekenhuis te Geldrop; Ziekenhuis Bethesda te Hoogeveen; GGZ Oost Brabant te Veghel en Oss; Catharina-Ziekenhuis te Eindhoven; en St. Jans Gasthuis te Weert.

De Maarten Kappelle stichting en Stichting De Drie Lichten wil ik bedanken voor de financiële steun van dit promotieonderzoek. De Nederlandse vereniging voor Slaap-Waak Onderzoek (NSWO), de Commissie Onderzoek en Ontwikkeling 
Kempenhaeghe en het Instituut Hersenen en Gedrag en Bureau Onderzoek Faculteit der Geneeskunde van de Universiteit Maastricht wil ik bedanken voor de financiële steun voor het drukken van dit proefschrift. Pharma Nord wil ik bedanken voor het beschikbaar stellen van medicatie voor het onderzoek. Het Universiteitsfonds Limburg/Stichting Wetenschappelijk Onderwijs Limburg (SWOL), Epilepsie-centrum Kempenhaeghe, Instituut Hersenen en Gedrag van Universiteit Maastricht en de afdeling neurologie van het Academisch Ziekenhuis Maastricht ben ik erkentelijk voor het financieren van mijn congresbezoeken in het buitenland.

Verder ben ik ben de Onderzoeksschool Neurowetenschappen Amsterdam dankbaar voor het fantastische opleidingsprogramma met cursussen, lezingen, en practica, en Stichting Balans, de Nederlandse vereniging voor ouders van kinderen met leer-, ontwikkelings- en gedragsstoornissen, voor het kenbaar maken van ons onderzoek via de website en Balans Belang.

Tot slot wil ik mijn vrienden en familie bedanken voor alle steun en liefde. In het bijzonder wil ik mijn ouders Boudewijn en Marianne bedanken. Jullie waren er altijd op de achtergrond met alle steun en vertrouwen en hebben het fundament gelegd voor de weg hiernaar toe. Kasper en Nienke, mijn dierbare broer en vriendin, geweldig dat jullie mij als paranimf willen bijstaan bij de laatste zware loodjes! Lieve Nienke, ik noem jou als laatste maar je vervulde een hoofdrol. Bij jou vond ik de momenten van rust, warmte en bezinning wanneer ik die nodig had. Onmisbaar was jouw wijze raad en ook de hulp die je als neuropsycholoog goed kon geven. Het geheel is meer dan de som der delen, het is een cliché maar zo is het. 


\section{Curriculum vitae}


Kristiaan Boudewijn van der Heijden werd geboren op 14 april 1975 te Delft. In 1993 behaalde hij het eindexamen VWO aan het Sint Stanislas College te Delft. In de zomer van 1993 deed hij een aanvullend eindexamen natuurkunde bij het James Boswell Instituut te Utrecht om vervolgens een jaar geneeskunde te studeren aan het Rijks Universitair Centrum te Antwerpen. In 1994 startte hij met de studie psychologie aan de Rijks Universiteit Leiden en vervolgde in 1996 met de afstudeerrichtingen Klinische Psychobiologie en Neuropsychologie (KPN) en Psychonomie bij de Universiteit van Amsterdam. Het afstudeeronderzoek "Effects of time and frequency uncertainty on signal detection" werd uitgevoerd in 19992000 bij prof. dr. Hafter aan de University of California, Berkeley in de Verenigde Staten. $\mathrm{Na}$ afronding van de literatuurscriptie over de biologische achtergrond van autisme spectrum stoornissen in 2000 behaalde hij in hetzelfde jaar bij de Universiteit van Amsterdam het doctoraal (Master of Arts) diploma psychologie (judicium "met genoegen") en de basisaantekening Psychodiagnostiek van het Nederlands Instituut van Psychologen (NIP). In augustus 2001 begon hij bij het Academisch Medisch Centrum te Amsterdam aan het promotieonderzoek "Chronic sleep onset insomnia in children with attention-deficit/hyperactivity disorder" bij promotor prof. dr. W.B. Gunning en copromotor dr. M.G. Smits. Het promotieonderzoek werd afgerond aan de Universiteit Maastricht en Epilepsiecentrum Kempenhaeghe Heeze met als toegevoegde promotoren prof. dr. J.S. Vles en prof. dr. A.P. Aldenkamp. Hij verliep het onderwijsprogramma van de Onderzoeksschool Neurowetenschappen Amsterdam (ONWA) en heeft op diverse internationale congressen lezingen gegeven over slaapstoornissen en behandeling met melatonine. 
List of publications 
Van der Heijden, K.B., Smits, M.G., Gunning, W.B. Sleep hygiene and actigraphically evaluated sleep characteristics in children with attentiondeficit/hyperactivity disorder and chronic sleep onset insomnia. I Sleep Res., 2006, 15: 53-60.

Van der Heijden, K.B., Blok, M.J., Spee, K., Archer, S.N., Smits, M.G., Curfs, L.M., Gunning, W.B. No evidence to support an association of PER3 clock gene polymorphism with ADHD-related idiopathic chronic sleep onset insomnia. Biol Rbythm Res, 2005, 36: 381-388.

Van der Heijden, K.B., Smits, M.G., Van Someren, E.J.W., Gunning, W.B. Idiopathic chronic sleep onset insomnia in attention-deficit/hyperactivity disorder: a circadian rhythm sleep disorder. Chronobiol Int., 2005, 22: 559-570.

Van der Heijden, K.B., Smits, M.G., Gunning, W.B. Sleep-related Disorders in ADHD; A review. Clin. Pediatr., 2005, 44: 201-210.

Van der Heijden, K.B., Smits, M.G., Van Someren, E.J.W., Gunning, W.B. Prediction of melatonin efficacy by pretreatment dim light melatonin onset in children with idiopathic chronic sleep onset insomnia. J Sleep Res., 2005, 14: 187-94.

Van der Heijden, K.B., Smits, M.G., Van Someren, E.J.W., Gunning, W.B. Childhood chronic sleep onset insomnia and late sleep onset: What's the difference? J Sleep Res., 2005, 14: 197-199.

Van der Heijden, K.B., Smits, M.G., Van Someren, E.J.W., Gunning, W.B. Idiopathic chronic sleep onset insomnia in attention-deficit/hyperactivity disorder: a circadian rhythm sleep disorder. Annual Volume Sleep-Wake Research in The Netherlands, 2005, 16: 71-74.

Van der Heijden K.B., Van Geijlswijk I.M., Smits M.G. Reactie op 'Verbetert melatonine de gezondheidstoestand en de slaap bij kinderen met een idiopathische chronische inslaapstoornis?' Tijdschrift voor Psycbiatrie, 2005, 47: 813-819.

Smits, M.G., Van Stel, H.F., Van der Heijden, K.B., Meijer, A.M., Coenen, A.M.L., Kerkhof, G.A. Melatonin improves health status and sleep in children with idiopathic chronic sleep-onset insomnia: a randomized placebo-controlled trial. $J$ Am Acad Child Adolesc Psychiatry. 2003, 42: 1286-93. 
Van der Heijden, K.B., Smits, M.G., Gunning, W.B. Sleep onset: relationship between actigraphy and sleep log measurements in children with sleep onset insomnia. Annual Volume Sleep-Wake Research in The Netherlands, 2002, 13: 44-46.

Van der Heijden, K.B., Smits, M.G., Veenboer, W., Vlasveld, L., Gerver, J.A.M., Gunning, W.B. Sleep onset insomnia in attention-deficit/hyperactivity disorder: stimulant use, psychiatric co-morbidity, family history, and other sleep-related problems. Submitted.

Van der Heijden, K.B., Smits, M.G., Van Someren, E.J.W., Ridderinkhof, K.R., Gunning, W.B. Effect of melatonin on sleep, behavior, cognitive performance, and quality of life in children with attention/deficit-hyperactivity disorder and chronic sleep onset insomnia. Revised manuscript resubmitted. 